\title{
The Sixth Painlevé Transcendents and the Associated Schlesinger Equation
}

\author{
by
}

\author{
Shun SHImomura
}

\begin{abstract}
For the Schlesinger equation associated with the sixth Painlevé equation (PVI) near the critical point, we present families of solutions expanded into convergent series with matrix coefficients. These families yield four basic solutions of (PVI) in Guzzetti's table describing the critical behaviours of the sixth Painlevé transcendents; two of the basic solutions are of complex power type, and two are of logarithmic type. Consequently, the convergence of the logarithmic solutions is verified. Furthermore we obtain more information on these basic solutions as well as on inverse logarithmic solutions. For complex power solutions, examining related inverse oscillatory ones, we discuss sequences of zeros and poles, non-decaying exponential oscillation and the analytic continuation around the critical point, and show the spiral distribution of poles conjectured by Guzzetti.
\end{abstract}

2010 Mathematics Subject Classification: Primary 34M55; Secondary 34M56, 34M35.

Keywords: Painlevé transcendents, Schlesinger equation, isomonodromy deformation, distribution of poles.

\section{$\S 1$. Introduction}

For the sixth Painlevé equation

$$
\begin{aligned}
\frac{d^{2} y}{d x^{2}}= & \frac{1}{2}\left(\frac{1}{y}+\frac{1}{y-1}+\frac{1}{y-x}\right)\left(\frac{d y}{d x}\right)^{2}-\left(\frac{1}{x}+\frac{1}{x-1}+\frac{1}{y-x}\right) \frac{d y}{d x} \\
& +\frac{y(y-1)(y-x)}{x^{2}(x-1)^{2}}\left(\alpha+\frac{\beta x}{y^{2}}+\frac{\gamma(x-1)}{(y-1)^{2}}+\frac{\delta x(x-1)}{(y-x)^{2}}\right)
\end{aligned}
$$

with $\alpha, \beta, \gamma, \delta \in \mathbb{C}$, Guzzetti [9] provided the tables of the critical behaviours of solutions as well as the parametric connection formulas, which are expected to give a complete description of the behaviours of the sixth Painlevé transcendents

Communicated by T. Mochizuki. Received November 20, 2014. Revised January 7, 2015.

S. Shimomura: Department of Mathematics, Keio University,

3-14-1, Hiyoshi, Kohoku-ku, Yokohama 223-8522, Japan;

e-mail: shimomur@math.keio.ac.jp

(C) 2015 Research Institute for Mathematical Sciences, Kyoto University. All rights reserved. 
as nonlinear special functions and to be of great use in applications to a variety of problems in mathematics and mathematical physics. All the types of solutions near $x=0$ listed in [9, Table 1] are derived through birational transformations from four basic solutions, two of which are of complex power type,

$$
\begin{aligned}
& y(x)=x\left(c_{1,-1}\left(a x^{\sigma}\right)^{-1}+c_{10}+a x^{\sigma}\right)+\sum_{n=2}^{\infty} x^{n} \sum_{m=-n}^{n} c_{n m}\left(a x^{\sigma}\right)^{m}, \\
& y(x)=x\left(c_{10}^{\prime}+a x^{\sigma_{0}}\right)+\sum_{n=2}^{\infty} x^{n} \sum_{m=0}^{n} c_{n m}^{\prime}\left(a x^{\sigma_{0}}\right)^{m}
\end{aligned}
$$

with

$$
\begin{aligned}
& c_{10}=\frac{1}{2 \sigma^{2}}\left(\sigma^{2}+\theta_{0}^{2}-\theta_{x}^{2}\right), \quad c_{1,-1}=\frac{1}{16 \sigma^{4}}\left(\left(\theta_{0}-\theta_{x}\right)^{2}-\sigma^{2}\right)\left(\left(\theta_{0}+\theta_{x}\right)^{2}-\sigma^{2}\right), \\
& c_{10}^{\prime}=\frac{\theta_{0}}{\theta_{0} \pm \theta_{x}}, \quad \sigma_{0}^{2}=\left(\theta_{0} \pm \theta_{x}\right)^{2},
\end{aligned}
$$

and two are of logarithmic type,

$$
\begin{array}{ll}
y(x)=x\left(\frac{\theta_{x}^{2}-\theta_{0}^{2}}{4}(a+\log x)^{2}+\frac{\theta_{0}^{2}}{\theta_{0}^{2}-\theta_{x}^{2}}\right)+\sum_{n=2}^{\infty} x^{n} P_{n}(\log x ; a) & \text { if } \theta_{0}^{2} \neq \theta_{x}^{2}, \\
y(x)=x\left(a \pm \theta_{0} \log x\right)+\sum_{n=2}^{\infty} x^{n} P_{n}(\log x ; a) & \text { if } \theta_{0}^{2}=\theta_{x}^{2} .
\end{array}
$$

Here $a$ and $\sigma$ are integration constants; $\theta_{0}$ and $\theta_{x}$ are numbers such that $\theta_{0}^{2}=-2 \beta$, $\theta_{x}^{2}=1-2 \delta$; and $P_{n}(\xi ; a)(n \geq 2)$ are polynomials in $\xi$.

The basic complex power solutions are represented by convergent series, which were obtained from the result in [5] combined with [8] (see also [12], [17]). Their convergence was proved in [5] by solving integral equations for the elliptic representation of (PVI) by successive approximations. As described in [8, $\S 7$, the inner sums $\sum_{m=-n}^{n} c_{n m}\left(a x^{\sigma}\right)^{m}$ are given by direct substitution into (PVI), and the determination of $c_{n m}$ is based on an overdetermined system of recursive relations, that is, for each $n$ it is necessary to check the compatibility of more than $2 n+1$ relations on $c_{n m}$. On the other hand, the leading terms of the basic logarithmic solutions were found in [6], [7] by applying a matching procedure to a Fuchsian system related to (PVI) through isomonodromy deformation (see also $\left[12,(1.9)^{\prime}\right]$ ). The polynomials $P_{n}(\xi ; a)$ are computed by direct substitution into (PVI), and in this case as well, the system of recursive relations is overdetermined. The convergence of the series for the logarithmic solutions, however, has not been proved, and no reference is made to $\operatorname{deg} P_{n}(\xi ; a)$ in the generic case except for a conjectural discussion in $[10, \S 3]$ related to the pole distribution of solutions of (PVI) with $(\alpha, \beta, \gamma, \delta)=\left((2 \mu-1)^{2} / 2,0,0,1 / 2\right)$. 
As applications of the convergent series representations for complex power solutions Guzzetti [8], [9], [10] derived oscillatory or inverse oscillatory behaviours and studied in detail sequences of poles accumulating at $x=0$ asymptotically along rays. Furthermore the analytic continuation of complex power solutions around $x=0$ was clarified through monodromy data in [4] and [5], which suggested the spiral distribution of poles.

Equation (PVI) is equivalent to the Schlesinger equation that is a deformation equation for the Fuchsian system mentioned above. For the Schlesinger equations corresponding to general Fuchsian systems, under a certain restriction on the eigenvalues of related matrices, Sato, Miwa and Jimbo [16] presented asymptotic estimates for solutions as well as their asymptotic expansions (for asymptotic estimates in a more general case see [4]). These estimates were used in the study of (PVI) (cf. [4], [5], [8], [12]). This fact suggests the possibility of deriving the formulas in Guzzetti's table by solving the Schlesinger equation that is equivalent to (PVI).

In view of the importance of the basic solutions, it is necessary to verify the convergence of the expansions in the logarithmic case as well. In the derivation of the series expansions of both types, it is preferable to avoid the use of overdetermined systems of recursive relations if possible, since it is not easy to check compatibility. In this paper we construct families of solutions of the Schlesinger equation associated with (PVI) expanded into convergent series with matrix coefficients, whose expressions are different from the asymptotic expansions in [16], and we derive the basic solutions of (PVI) without dealing with overdetermined recusive relations. Consequently, the convergence of the logarithmic solutions is shown, and more information on the expansions is obtained; for example, we may fix the bound of $\operatorname{deg} P_{n}(\xi ; a)$, which enables us to get more detailed expressions for inverse logarithmic solutions including the Chazy solutions [14].

Our results on the solutions of the Schlesinger equation and on the basic solutions of (PVI) are stated in Section 2.1. The inverse logarithmic solutions in Corollary 2.5 follow from the basic logarithmic ones. In Section 2.2, for complex power solutions, using the representations of them and of related inverse oscillatory ones, we observe how they behave and get results describing sequences of zeros and poles, non-decaying exponential oscillation and the analytic continuation around $x=0$. We discuss the analytic continuation by matching, which shows the spiral distribution of poles conjectured by Guzzetti [4], [5]. In Section 3 we describe a relation between (PVI) and the Schlesinger equation in our case (for isomonodromy deformation related to (PVI) see, for example, [2], [4], [5], [13], [14]). The convergent solutions of the Schlesinger equation are constructed in Section 5 by iteration on a certain kind of rings of formal or convergent series with matrix co- 
efficients, which are defined in Section 4. In our argument, which is similar to that in [17], we define a suitable norm in each ring, and, instead of the contour integral, consider an operator acting in it that assigns the formal primitive function of a related series. The final section is devoted to the derivation of the basic solutions of (PVI).

Throughout this paper we use the following symbols:

(1) for a ring $\mathbb{A}, M_{2}(\mathbb{A})$ is the ring of $2 \times 2$ matrices $C=\left(C_{i j}\right)$ with $C_{i j} \in \mathbb{A}$ $(i, j=1,2)$, and $G L_{2}(\mathbb{A}):=\left\{C \in M_{2}(\mathbb{A}) ; C^{-1} \in M_{2}(\mathbb{A})\right\} ;$

(2) $I, J, \Delta, \Delta_{-}$denote the matrices

$$
I=\left(\begin{array}{cc}
1 & 0 \\
0 & 1
\end{array}\right), \quad J=\left(\begin{array}{cc}
1 & 0 \\
0 & -1
\end{array}\right), \quad \Delta=\left(\begin{array}{ll}
0 & 1 \\
0 & 0
\end{array}\right), \quad \Delta_{-}=\left(\begin{array}{ll}
0 & 0 \\
1 & 0
\end{array}\right)
$$

(3) $\mathcal{R}(\mathbb{C} \backslash\{0\})$ denotes the universal covering of $\mathbb{C} \backslash\{0\}$;

(4) $\mathbb{C}_{\theta}:=\mathbb{C}\left[\theta_{0}, \theta_{x}, \theta_{1}, \theta_{\infty}, \theta_{\infty}^{-1}\right],\left(\theta_{\iota}\right):=\left(\theta_{0}, \theta_{x}, \theta_{1}, \theta_{\infty}\right)$ (cf. (2.2), (2.3));

(5) the following are rings of formal series $\Phi$ (cf. Section 4$)$ :

$$
\begin{aligned}
\widehat{\mathfrak{L}}: & \Phi=\sum_{n=1}^{\infty} \sum_{m=0}^{2 n} C_{m}^{n}(\kappa t)^{n} \log ^{m} t, & C_{m}^{n} \in M_{2}\left(\mathbb{C}_{\theta}\right), \\
\widehat{\mathfrak{L}}^{*}: & \Phi=\sum_{n=1}^{\infty} \sum_{m=0}^{n} C_{m}^{n}(\kappa t)^{n} \log ^{m} t, & C_{m}^{n} \in M_{2}\left(\mathbb{C}_{\theta}\right), \\
\widehat{\mathfrak{S}}: & \Phi=\sum_{n=1}^{\infty} \sum_{m=-n}^{n} C_{m}^{n}(\kappa t)^{n} t^{\sigma m}, & C_{m}^{n} \in M_{2}\left(\mathbb{C}_{\theta}(\sigma)\right), \\
\widehat{\mathfrak{S}}^{+}\left(\sigma_{0}\right): & \Phi=\sum_{n=1}^{\infty} \sum_{m=0}^{n} C_{m}^{n}(\kappa t)^{n} t^{\sigma_{0} m}, &
\end{aligned}
$$

and $\mathfrak{L}(D), \mathfrak{S}(D(\Sigma)), \mathfrak{S}^{+}\left(D\left(\sigma_{0}\right)\right)$ denote the subrings consisting of $\Phi$ such that $\|\Phi\|<\infty$ in the domains $D, D(\Sigma), D\left(\sigma_{0}\right)$, respectively.

\section{§2. Results}

\section{$\S 2.1$. Solutions of the Schlesinger equation and (PVI)}

The Schlesinger equation

$$
\begin{aligned}
x \frac{d A_{0}}{d x} & =\left[A_{x}, A_{0}\right], \quad x \frac{d A_{x}}{d x}=\left[A_{0}, A_{x}\right]-\frac{x}{1-x}\left[A_{1}, A_{x}\right], \\
x \frac{d A_{1}}{d x} & =-\frac{x}{1-x}\left[A_{x}, A_{1}\right],
\end{aligned}
$$


where $A_{0}, A_{x}, A_{1}$ are $2 \times 2$ unknown matrices, controls the isomonodromy deformation of a Fuchsian system that will be mentioned in the next section, and is equivalent to (PVI) under certain conditions.

Let $\theta_{0}, \theta_{x}, \theta_{1}, \theta_{\infty}$ be complex parameters such that

$$
\alpha=\left(\theta_{\infty}-1\right)^{2} / 2, \quad-\beta=\theta_{0}^{2} / 2, \quad \gamma=\theta_{1}^{2} / 2, \quad 1 / 2-\delta=\theta_{x}^{2} / 2, \quad \theta_{\infty} \neq 0,
$$

where $\alpha, \beta, \gamma, \delta$ are the coefficients of (PVI), and set

$$
\mathbb{C}_{\theta}:=\mathbb{C}\left[\theta_{0}, \theta_{x}, \theta_{1}, \theta_{\infty}, \theta_{\infty}^{-1}\right] .
$$

For a given number $b_{0}>0$, suppose that $\left(\theta_{\iota}\right):=\left(\theta_{0}, \theta_{x}, \theta_{1}, \theta_{\infty}\right)$ satisfies

$$
\left|\theta_{0}\right|+\left|\theta_{x}\right|+\left|\theta_{1}\right|+\left|\theta_{\infty}\right|+\left|\theta_{\infty}^{-1}\right|<b_{0} .
$$

Suppose that $\Lambda_{0}, \Lambda_{x}, \Lambda_{1} \in M_{2}\left(\mathbb{C}_{\theta}\left[\sigma, \sigma^{-1}\right]\right), T \in G L_{2}\left(\mathbb{C}_{\theta}\left[\sigma, \sigma^{-1}\right]\right)$ and $\Lambda:=\Lambda_{0}+\Lambda_{x}$ have the properties:

(P.1) the eigenvalues of $\Lambda_{0}, \Lambda_{x}, \Lambda_{1}$ are $\pm \theta_{0} / 2, \pm \theta_{x} / 2, \pm \theta_{1} / 2$, respectively;

(P.2) $T^{-1} \Lambda T=(\sigma / 2) J$

(P.3) $\Lambda_{0}+\Lambda_{x}+\Lambda_{1}=-\left(\theta_{\infty} / 2\right) J$.

Then we have

Theorem 2.1. (1) Let $\Sigma_{0}$ be a bounded domain such that

$$
\Sigma_{0} \subset \mathbb{C} \backslash S_{0} \quad \text { with } \quad S_{0}:=\{\sigma \leq-1\} \cup\{0\} \cup\{\sigma \geq 1\} \subset \mathbb{R}
$$

and $\operatorname{dist}\left(\Sigma_{0}, S_{0}\right)>0$. Then (2.1) admits a two-parameter family of solutions

$$
\left\{\left(A_{0}(\sigma, \rho, x), A_{x}(\sigma, \rho, x), A_{1}(\sigma, \rho, x)\right) ;(\sigma, \rho) \in \Sigma_{0} \times(\mathbb{C} \backslash\{0\})\right\}
$$

given by the convergent series

$$
\begin{aligned}
& A_{0}(\sigma, \rho, x)=\left(\rho x^{\sigma}\right)^{\Lambda / \sigma}\left(\Lambda_{0}+\sum_{n=1}^{\infty} x^{n} \Pi_{0}^{n}\left(\sigma, \rho x^{\sigma}\right)\right)\left(\rho x^{\sigma}\right)^{-\Lambda / \sigma} \\
& A_{x}(\sigma, \rho, x)=\left(\rho x^{\sigma}\right)^{\Lambda / \sigma}\left(\Lambda_{x}+\sum_{n=1}^{\infty} x^{n} \Pi_{x}^{n}\left(\sigma, \rho x^{\sigma}\right)\right)\left(\rho x^{\sigma}\right)^{-\Lambda / \sigma} \\
& A_{1}(\sigma, \rho, x)=\Lambda_{1}+\sum_{n=1}^{\infty} x^{n} \Pi_{1}^{n}\left(\sigma, \rho x^{\sigma}\right)
\end{aligned}
$$

with

$$
\Pi_{\iota}^{n}(\sigma, \xi)=\sum_{m=-n}^{n} C_{\iota m}^{n}(\sigma) \xi^{m}, \quad C_{\iota m}^{n}(\sigma) \in M_{2}\left(\mathbb{C}_{\theta}(\sigma)\right)
$$


$(\iota=0, x, 1)$, which are holomorphic in $(\sigma, \rho, x) \in \Omega\left(\Sigma_{0}, \varepsilon_{0}\right) \subset \Sigma_{0} \times(\mathbb{C} \backslash\{0\}) \times$ $\mathcal{R}(\mathbb{C} \backslash\{0\})$. Here

$$
\begin{aligned}
\Omega\left(\Sigma_{0}, \varepsilon_{0}\right) & :=\bigcup_{(\sigma, \rho) \in \Sigma_{0} \times(\mathbb{C} \backslash\{0\})}\{(\sigma, \rho)\} \times \Omega_{\sigma, \rho}\left(\varepsilon_{0}\right), \\
\Omega_{\sigma, \rho}\left(\varepsilon_{0}\right) & :=\left\{x \in \mathcal{R}(\mathbb{C} \backslash\{0\}) ;|x|<\varepsilon_{0},\left|x\left(\rho x^{\sigma}\right)\right|<\varepsilon_{0},\left|x\left(\rho x^{\sigma}\right)^{-1}\right|<\varepsilon_{0}\right\},
\end{aligned}
$$

$\varepsilon_{0}=\varepsilon_{0}\left(\Sigma_{0}, b_{0}\right)$ being a sufficiently small positive number depending only on $\Sigma_{0}$ and $b_{0}$.

(2) If $\left(T^{-1} \Lambda_{0} T\right)_{21}$ vanishes at

$$
\sigma=\sigma_{0} \in \Sigma_{+}:=\mathbb{C} \backslash(\{\sigma \leq-1\} \cup \mathbb{Z}),
$$

then (2.1) admits a one-parameter family of solutions

$$
\left\{\left(A_{0}\left(\sigma_{0}, \rho, x\right), A_{x}\left(\sigma_{0}, \rho, x\right), A_{1}\left(\sigma_{0}, \rho, x\right)\right) ; \rho \in \mathbb{C}\right\}
$$

given by the representations above restricted to $\sigma=\sigma_{0}$ whose inner sums satisfy $\Pi_{1}^{n}\left(\sigma_{0}, \xi\right)=\sum_{m=0}^{n} C_{1 m}^{n}\left(\sigma_{0}\right) \xi^{m}$ and, for $\iota=0, x$,

$\xi^{\Lambda / \sigma_{0}} \Pi_{\iota}^{n}\left(\sigma_{0}, \xi\right) \xi^{-\Lambda / \sigma_{0}}=\sum_{m=0}^{n+1} \tilde{C}_{\iota m}^{n}\left(\sigma_{0}\right) \xi^{m}, \quad \xi^{\Lambda / \sigma_{0}} \Lambda_{\iota} \xi^{-\Lambda / \sigma_{0}}=\tilde{C}_{\iota 0}^{0}\left(\sigma_{0}\right)+\tilde{C}_{\iota 1}^{0}\left(\sigma_{0}\right) \xi$ with $\tilde{C}_{\iota m}^{n}\left(\sigma_{0}\right) \in M_{2}\left(\mathbb{C}_{\theta}\left(\sigma_{0}\right)\right)(n \geq 0)$. For $\iota=0, x, 1, A_{\iota}\left(\sigma_{0}, \rho, x\right)$ are holomorphic in $(\rho, x) \in \Omega\left(\varepsilon_{0}\right) \subset \mathbb{C} \times \mathcal{R}(\mathbb{C} \backslash\{0\})$, where

$$
\begin{aligned}
\Omega\left(\varepsilon_{0}\right) & :=\bigcup_{\rho \in \mathbb{C}}\{\rho\} \times \Omega_{\rho}\left(\varepsilon_{0}\right), \\
\Omega_{\rho}\left(\varepsilon_{0}\right) & :=\left\{x \in \mathcal{R}(\mathbb{C} \backslash\{0\}) ;|x|<\varepsilon_{0},\left|x\left(\rho x^{\sigma_{0}}\right)\right|<\varepsilon_{0}\right\},
\end{aligned}
$$

$\varepsilon_{0}=\varepsilon_{0}\left(\sigma_{0}, b_{0}\right)$ being a sufficiently small positive number depending only on $\sigma_{0}$ and $b_{0}$.

Remark 2.1. The poles of each entry of $C_{\iota m}^{n}(\sigma)$ belong to $\mathbb{Q} \backslash\{0<|\sigma|<1\}$.

Remark 2.2. By (P.2) each entry of $A_{0}(\sigma, \rho, x), A_{x}(\sigma, \rho, x)$ and $A_{1}(\sigma, \rho, x)$ is a power series in $x$ and $\left(\rho x^{\sigma}\right)^{ \pm 1}$, in particular, $\left(A_{0}\left(\sigma_{0}, 0, x\right), A_{x}\left(\sigma_{0}, 0, x\right), A_{1}\left(\sigma_{0}, 0, x\right)\right)$ is a Taylor series solution convergent for $|x|<\varepsilon_{0}$.

Suppose that $\Lambda_{0}, \Lambda_{x}, \Lambda_{1} \in M_{2}\left(\mathbb{C}_{\theta}\right), T \in G L_{2}\left(\mathbb{C}_{\theta}\right)$ and $\Lambda=\Lambda_{0}+\Lambda_{x}$ have the properties (P.1), (P.3) above and

$\left(\mathrm{P} .2^{\prime}\right) T^{-1} \Lambda T=\Delta$.

Then we have logarithmic solutions. 
Theorem 2.2. System (2.1) admits a one-parameter family of solutions

$$
\left\{\left(A_{0}(\rho, x), A_{x}(\rho, x), A_{1}(\rho, x)\right) ; \rho \in \mathcal{R}(\mathbb{C} \backslash\{0\})\right\}
$$

given by the convergent series

$$
\begin{aligned}
& A_{0}(\rho, x)=(\rho x)^{\Lambda}\left(\Lambda_{0}+\sum_{n=1}^{\infty} x^{n} \Pi_{0}^{* n}(\log (\rho x))\right)(\rho x)^{-\Lambda} \\
& A_{x}(\rho, x)=(\rho x)^{\Lambda}\left(\Lambda_{x}+\sum_{n=1}^{\infty} x^{n} \Pi_{x}^{* n}(\log (\rho x))\right)(\rho x)^{-\Lambda} \\
& A_{1}(\rho, x)=\Lambda_{1}+\sum_{n=1}^{\infty} x^{n} \Pi_{1}^{* n}(\log (\rho x))
\end{aligned}
$$

with

$$
\Pi_{\iota}^{* n}(\xi)=\sum_{m=0}^{2 n} C_{\iota m}^{* n} \xi^{m}, \quad C_{\iota m}^{* n} \in M_{2}\left(\mathbb{C}_{\theta}\right)
$$

$(\iota=0, x, 1)$, which are holomorphic in $(\rho, x) \in \Omega^{*}\left(\varepsilon_{0}, \Theta_{0}\right) \subset \mathcal{R}(\mathbb{C} \backslash\{0\})^{2}$. Here

$$
\begin{aligned}
& \Omega^{*}\left(\varepsilon_{0}, \Theta_{0}\right):=\bigcup_{\rho \in \mathcal{R}(\mathbb{C} \backslash\{0\})}\{\rho\} \times \Omega_{\rho}^{*}\left(\varepsilon_{0}, \Theta_{0}\right), \\
& \Omega_{\rho}^{*}\left(\varepsilon_{0}, \Theta_{0}\right):=\left\{x \in \mathcal{R}(\mathbb{C} \backslash\{0\}) ;|\rho x|<\varepsilon_{0},\left|x(\rho x)^{-1 / 2}\right|<\varepsilon_{0},|\arg (\rho x)|<\Theta_{0}\right\},
\end{aligned}
$$

$\Theta_{0}$ being a given positive number and $\varepsilon_{0}=\varepsilon_{0}\left(\Theta_{0}, b_{0}\right)$ a sufficiently small positive number depending only on $\Theta_{0}$ and $b_{0}$. Furthermore, if $\left(T^{-1} \Lambda_{0} T\right)_{21}=0$, then $\Pi_{1}^{* n}(\xi)=\sum_{m=0}^{n} C_{1 m}^{* n} \xi^{m}$ and, for $\iota=0, x$,

$$
e^{\Lambda \xi} \Pi_{\iota}^{* n}(\xi) e^{-\Lambda \xi}=\sum_{m=0}^{n+1} \tilde{C}_{\iota m}^{* n} \xi^{m}, \quad e^{\Lambda \xi} \Lambda_{\iota} e^{-\Lambda \xi}=\tilde{C}_{\iota 0}^{* 0}+\tilde{C}_{\iota 1}^{* 0} \xi,
$$

with $\tilde{C}_{\iota m}^{* n} \in M_{2}\left(\mathbb{C}_{\theta}\right)(n \geq 0)$.

From these solutions of the Schlesinger equation depending on suitably chosen matrices $\Lambda_{0}, \Lambda_{x}, \Lambda_{1}$ (cf. Lemmas 6.1 and 6.2), we obtain the following theorems on (PVI), in which $\Sigma_{0}, \Sigma_{+}, \Omega\left(\Sigma_{0}, \varepsilon\right), \Omega(\varepsilon)$ and $\Omega^{*}\left(\varepsilon, \Theta_{0}\right)$ are as in Theorems 2.1 and 2.2 .

Theorem 2.3. (1) Equation (PVI) admits a two-parameter family of solutions $\left\{y(\sigma, \rho, x) ;(\sigma, \rho) \in \Sigma_{0} \times(\mathbb{C} \backslash\{0\})\right\}$ given by the convergent series

$$
y(\sigma, \rho, x)=\sum_{n=1}^{\infty} x^{n} R_{n}\left(\sigma, \rho x^{\sigma}\right),
$$


which is holomorphic in $(\sigma, \rho, x) \in \Omega\left(\Sigma_{0}, \hat{\varepsilon}_{0}\right), \hat{\varepsilon}_{0}=\hat{\varepsilon}_{0}\left(\Sigma_{0}, b_{0}\right)$ being a sufficiently small positive number depending only on $\Sigma_{0}$ and $b_{0}$. Here

$$
R_{n}(\sigma, \xi)=\sum_{m=-n}^{n} c_{m}^{n}(\sigma) \xi^{m}, \quad c_{m}^{n}(\sigma) \in \mathbb{C}_{\theta}(\sigma)
$$

in particular,

$$
R_{1}(\sigma, \xi)=\xi+\frac{1}{2 \sigma^{2}}\left(\sigma^{2}+\theta_{0}^{2}-\theta_{x}^{2}\right)+\frac{1}{16 \sigma^{4}}\left(\left(\theta_{0}-\theta_{x}\right)^{2}-\sigma^{2}\right)\left(\left(\theta_{0}+\theta_{x}\right)^{2}-\sigma^{2}\right) \xi^{-1} .
$$

(2) If $\sigma_{0}^{2}=\left(\theta_{0} \pm \theta_{x}\right)^{2}$ and if $\sigma_{0} \in \Sigma_{+}$, then (PVI) admits a one-parameter family of solutions $\left\{y_{+}\left(\sigma_{0}, \rho, x\right) ; \rho \in \mathbb{C}\right\}$ given by the convergent series

$$
y_{+}\left(\sigma_{0}, \rho, x\right)=\sum_{n=1}^{\infty} x^{n} R_{n}\left(\sigma_{0}, \rho x^{\sigma_{0}}\right), \quad R_{n}\left(\sigma_{0}, \xi\right)=\sum_{m=0}^{n} c_{m}^{n}\left(\sigma_{0}\right) \xi^{m},
$$

which is holomorphic in $(\rho, x) \in \Omega\left(\varepsilon_{0}^{+}\right), \varepsilon_{0}^{+}=\varepsilon_{0}^{+}\left(\sigma_{0}, b_{0}\right)$ being a sufficiently small positive number depending only on $\sigma_{0}$ and $b_{0}$.

As will be shown in Section 2.2, the series expansions given above allow for a variety of behaviours of $y(\sigma, \rho, x)$ and $y_{+}\left(\sigma_{0}, \rho, x\right)$.

Theorem 2.4. Equation (PVI) has a one-parameter family of solutions $\left\{y_{0}(\rho, x)\right.$; $\rho \in \mathcal{R}(\mathbb{C} \backslash\{0\})\}$ given by the convergent series

$$
y_{0}(\rho, x)=\sum_{n=1}^{\infty} x^{n} P_{n}(\log (\rho x)),
$$

which is holomorphic in $(\rho, x) \in \Omega^{*}\left(\varepsilon_{0}^{0}, \Theta_{0}\right), \varepsilon_{0}^{0}=\varepsilon_{0}^{0}\left(\Theta_{0}, b_{0}\right)$ being a sufficiently small positive number depending only on $\Theta_{0}$ and $b_{0}$. Here

$$
P_{n}(\xi)=\sum_{m=0}^{2 n} c_{m}^{* n} \xi^{m}, \quad c_{m}^{* n} \in \mathbb{C}_{\theta}
$$

in particular,

$$
P_{1}(\xi)=\frac{1}{4}\left(\theta_{x}^{2}-\theta_{0}^{2}\right) \xi^{2} \pm \theta_{0} \xi
$$

If $\theta_{x}^{2}=\theta_{0}^{2}$, then $P_{n}(\xi)=\sum_{m=0}^{n} c_{m}^{* n} \xi^{m}$ for every $n \geq 1$.

Remark 2.3. In Theorems 2.3 and 2.4, $\mathbb{C}_{\theta}(\sigma)$ and $\mathbb{C}_{\theta}$ may be replaced by $\mathbb{Q}_{\theta}(\sigma)$ and $\mathbb{Q}_{\theta}$, respectively, where $\mathbb{Q}_{\theta}:=\mathbb{Q}\left[\theta_{0}, \theta_{x}, \theta_{1}, \theta_{\infty}, \theta_{\infty}^{-1}\right]$, because the entries of the matrices in Lemmas 6.1 and 6.2 belong to $\mathbb{Q}_{\theta}(\sigma)$. 
Remark 2.4. Each solution as a double series converges absolutely and uniformly on every compact set contained in the corresponding domain. For example $\sum_{n=1}^{\infty} \sum_{m=-n}^{n}\left|c_{m}^{n}(\sigma)\right|\left|x^{n}\right|\left|\left(\rho x^{\sigma}\right)^{m}\right|$ for $y(\sigma, \rho, x)$ converges uniformly on every compact set contained in $\Omega\left(\Sigma_{0}, \hat{\varepsilon}_{0}\right)$.

Remark 2.5. The solutions $y(\sigma, \rho, x), y_{+}\left(\sigma_{0}, \rho, x\right)$ and $y_{0}(\rho, x)$ agree with the basic solutions $[9,(36)],[9,(41)]$ and $[9,(48),(44)]$, respectively.

Remark 2.6. Theorem 2.3 gives the Taylor series solution $y_{+}\left(\sigma_{0}, 0, x\right)$ as well, which agrees with $[9,(42)]$.

Remark 2.7. (1) If $\theta_{x}^{2}=\theta_{0}^{2}$, then $P_{n}(\xi)$ may be written as $P_{n}(\xi)=\tilde{P}_{n}\left( \pm \theta_{0} \xi\right)$ with $\tilde{P}_{n}(\tilde{\xi}) \in \mathbb{C}_{\theta}[\tilde{\xi}]$ for $\left(\theta_{\iota}\right)$ satisfying (2.4) (cf. Remark 6.2$)$, so that $y_{0}(\rho, x)$ admits another expression of the form

$$
\tilde{y}_{0}(a, x)=\sum_{n=1}^{\infty} x^{n} \tilde{P}_{n}\left(a \pm \theta_{0} \log x\right),
$$

$a$ being an integration constant. Hence, under the condition $\theta_{x}=\theta_{0}=0$, this yields the Taylor series solution $\left.\tilde{y}_{0}(a, x)\right|_{\theta_{0}=0}=\left.\sum_{n=1}^{\infty} \tilde{P}_{n}(a)\right|_{\theta_{0}=0} x^{n}$, which agrees with $[9,(46)]$.

(2) If $\theta_{x}^{2} \neq \theta_{0}^{2}$, setting $\rho=e^{a} \exp \left(\mp 2 \theta_{0} /\left(\theta_{x}^{2}-\theta_{0}^{2}\right)\right)$ we have $P_{1}(\log (\rho x))=$ $\left(\theta_{x}^{2}-\theta_{0}^{2}\right)(a+\log x)^{2} / 4-\theta_{0}^{2} /\left(\theta_{x}^{2}-\theta_{0}^{2}\right)$ as in $[9,(48)]$.

Remark 2.8. The logarithmic solution with $\theta_{x}^{2}=\theta_{0}^{2}$ as well as those with $\theta_{x} \pm \theta_{0}$ $\in \mathbb{Z}[9,(43)]$ derived through birational transformations may also be obtained from the polynomial Hamiltonian system

$$
d y / d x=\partial H_{\mathrm{VI}} / \partial z, \quad d z / d x=-\partial H_{\mathrm{VI}} / \partial y,
$$

where

$$
\begin{aligned}
H_{\mathrm{VI}}= & \frac{1}{x(x-1)}\left(y(y-1)(y-x) z^{2}\right. \\
& \left.\quad-\left(\theta_{0}(y-1)(y-x)+\theta_{1} y(y-x)+\left(\theta_{x}-1\right) y(y-1)\right) z+\theta_{\sharp}(y-x)\right), \\
\theta_{\sharp}= & \frac{1}{4}\left(\left(\theta_{0}+\theta_{x}+\theta_{1}-1\right)^{2}-\theta_{\infty}^{2}\right) \quad(\text { see }[15],[11]) .
\end{aligned}
$$

As a corollary of Theorem 2.4 we obtain inverse logarithmic solutions including the Chazy solutions (cf. [9, (60), (64)], [1], [3], [14]) by using the birational transformation [15]

$$
\theta_{0} \mapsto \theta_{\infty}-1, \quad \theta_{x} \mapsto \theta_{1}, \quad \theta_{1} \mapsto \theta_{x}, \quad \theta_{\infty} \mapsto \theta_{0}+1, \quad y(x) \mapsto x / y(x) .
$$


Corollary 2.5. Instead of (2.4), suppose that $\left|\theta_{0}\right|+\left|\theta_{x}\right|+\left|\theta_{1}\right|+\left|\theta_{\infty}\right|+\left|\left(\theta_{0}+1\right)^{-1}\right|$ $<\tilde{b}_{0}$, where $\tilde{b}_{0}$ is a given positive number. Then (PVI) admits a one-parameter family of solutions $\left\{y_{0}^{\text {inverse }}(\rho, x) ; \rho \in \mathcal{R}(\mathbb{C} \backslash\{0\})\right\}$ given by the convergent series

$$
y_{0}^{\text {inverse }}(\rho, x)=\sum_{n=0}^{\infty} x^{n} Q_{n}(\log (\rho x)),
$$

which is holomorphic in $(\rho, x) \in \Omega^{*}\left(\tilde{\varepsilon}_{0}, \Theta_{0}\right), \tilde{\varepsilon}_{0}=\tilde{\varepsilon}_{0}\left(\Theta_{0}, \tilde{b}_{0}\right)$ being a sufficiently small positive number depending only on $\Theta_{0}$ and $\tilde{b}_{0}$. Here $Q_{n}(\xi)(n \geq 0)$ are functions with the properties:

(i) if $\theta_{1}^{2} \neq\left(\theta_{\infty}-1\right)^{2}$, then

$$
Q_{n}(\xi)=\xi^{2 n-2} \sum_{m=0}^{\infty} \tilde{c}_{m}^{n} \xi^{-m}, \quad \tilde{c}_{m}^{n} \in \mathbb{C}_{\tilde{\theta}}\left[\left(\theta_{1}^{2}-\left(\theta_{\infty}-1\right)^{2}\right)^{-1}\right],
$$

with $\mathbb{C}_{\tilde{\theta}}:=\mathbb{C}\left[\theta_{0}, \theta_{x}, \theta_{1}, \theta_{\infty},\left(\theta_{0}+1\right)^{-1}\right]$, which are holomorphic for $|\xi|>\xi_{\infty}$, in particular,

$$
Q_{0}(\xi)=\frac{4 \xi^{-2}}{\theta_{1}^{2}-\left(\theta_{\infty}-1\right)^{2}}\left(1 \pm \frac{4\left(\theta_{\infty}-1\right) \xi^{-1}}{\theta_{1}^{2}-\left(\theta_{\infty}-1\right)^{2}}\right)^{-1},
$$

$\xi_{\infty}$ being a sufficiently large positive number independent of $n$;

(ii) if $\theta_{1}= \pm\left(\theta_{\infty}-1\right) \neq 0$, then

$$
Q_{n}(\xi)=\xi^{n-1} \sum_{m=0}^{2 n} \tilde{c}_{m}^{n} \xi^{-m}, \quad \tilde{c}_{m}^{n} \in \mathbb{C}_{\tilde{\theta}}\left[\left(\theta_{\infty}-1\right)^{-1}\right]
$$

in particular, $Q_{0}(\xi)= \pm \xi^{-1} /\left(\theta_{\infty}-1\right)$.

$$
\S 2.2 \text {. Behaviours of } y(\sigma, \rho, x) \text { and } y_{+}\left(\sigma_{0}, \rho, x\right)
$$

As shown in [4], [5], [9], a complex power solution has a dominant term that changes according to the integration constant $\sigma$. Let us examine the behaviours of $y(\sigma, \rho, x)$ and $y_{+}\left(\sigma_{0}, \rho, x\right)$ in Theorem 2.3. If $\operatorname{Im} \sigma=0$, then, in $\Omega_{\sigma, \rho}\left(\hat{\varepsilon}_{0}\right), y(\sigma, \rho, x) \sim \rho x^{1+\sigma}$ if $-1<\sigma<0$, and $y(\sigma, \rho, x) \sim c_{-1}^{1}(\sigma) \rho^{-1} x^{1-\sigma}$ if $0<\sigma<1$ and $c_{-1}^{1}(\sigma) \neq 0$.

In what follows we suppose that $\operatorname{Im} \sigma \neq 0$, and observe $y(\sigma, \rho, x)$ along the curve

$$
\Gamma\left(r_{0}, \omega\right):(1+\operatorname{Re} \sigma-\omega) \log |x|-\operatorname{Im} \sigma \cdot \arg x=r_{0}
$$

with $0 \leq \omega \leq 2, r_{0} \in \mathbb{R}$, which was considered in [4], [5]. This is a ray tending to $x=0$ if $1+\operatorname{Re} \sigma-\omega=0$, and a spiral curve if $1+\operatorname{Re} \sigma-\omega \neq 0$. Note that $\left|x^{1+\sigma}\right|=e^{r_{0}}|x|^{\omega},\left|x^{1-\sigma}\right|=e^{-r_{0}}|x|^{2-\omega}$ along $\Gamma\left(r_{0}, \omega\right)$. 
2.2.1. Dominant terms. For each $\omega \neq 0,1,2, y(\sigma, \rho, x)$ along $\Gamma\left(r_{0}, \omega\right)$ has a dominant term.

Proposition 2.6. For every $r_{0} \in \mathbb{R}$, along $\Gamma\left(r_{0}, \omega\right), y(\sigma, \rho, x) \sim \rho x^{1+\sigma}$ if $0<$ $\omega<1$, and $y(\sigma, \rho, x) \sim c_{-1}^{1}(\sigma) \rho^{-1} x^{1-\sigma}$ if $1<\omega<2$ and $c_{-1}^{1}(\sigma) \neq 0$.

2.2.2. Trigonometric oscillation. Suppose that $\omega=1$. Let $\sigma$ and $\rho$ be fixed. Along $\Gamma\left(r_{0}, 1\right)$,

$$
x^{\sigma}=e^{r_{0}} \exp \left(i(\operatorname{Im} \sigma)^{-1}\left(|\sigma|^{2} \log |x|-r_{0} \operatorname{Re} \sigma\right)\right) .
$$

Then $R_{1}\left(\sigma, \rho x^{\sigma}\right)$ has three balanced terms, and is written in the form $R_{1}\left(\sigma, \rho x^{\sigma}\right)=$ $\rho x^{-\sigma}\left(x^{\sigma}-\xi_{0}^{-}\right)\left(x^{\sigma}-\xi_{0}^{+}\right)$with

$$
\xi_{0}^{ \pm}=\xi_{0}^{ \pm}(\sigma, \rho):=\frac{1}{4 \sigma^{2} \rho}\left(\theta_{x}^{2}-\left(\theta_{0} \pm \sigma\right)^{2}\right) .
$$

Let us write $y(\sigma, \rho, x)=x R_{1}\left(\sigma, \rho x^{\sigma}\right)+x^{2} R_{*}(x)$ or

$$
\begin{aligned}
\rho^{-1} x^{\sigma-1} y(\sigma, \rho, x) & =\rho^{-1} x^{\sigma} R_{1}\left(\sigma, \rho x^{\sigma}\right)+\rho^{-1} x^{1+\sigma} R_{*}(x) \\
& =\left(x^{\sigma}-\xi_{0}^{-}\right)\left(x^{\sigma}-\xi_{0}^{+}\right)+\rho^{-1} x^{1+\sigma} R_{*}(x) .
\end{aligned}
$$

For any $x_{*} \in \Gamma\left(r_{0}, 1\right)$, if $\left|x-x_{*}\right|<\left|x_{*}\right| / 2$, then $\left|x^{\sigma}\right|,\left|x^{-\sigma}\right|=O(1)$, and hence $R_{*}(x)=O(1)$, the implied constants being independent of $x_{*}$. The last estimate may be verified by using $\left|c_{m}^{n}(\sigma)\right| \leq \epsilon^{-n}$ with some $\epsilon>0$, which follows from absolute convergence (cf. Remark 2.4).

Simple zeros. Suppose that $\theta_{0} \neq 0$, and that $\xi_{0}^{ \pm} \neq 0$. Then $\xi_{0}^{-}-\xi_{0}^{+} \neq 0$. Take $r_{0}=r_{0}^{-}=r_{0}^{-}(\sigma, \rho)$ such that $\exp \left(r_{0}^{-}\right)=\left|\xi_{0}^{-}\right|$, and set $\mu_{-}=\mu_{-}(\sigma, \rho)=\arg \xi_{0}^{-}$. Then

$$
x^{\sigma}-\xi_{0}^{-}=-2 i \exp \left(\left(\sigma \log x+r_{0}^{-}+i \mu_{-}\right) / 2\right) \sin \left(\left(i \sigma \log x+\mu_{-}-i r_{0}^{-}\right) / 2\right) .
$$

On $\Gamma\left(r_{0}^{-}, 1\right)$ this is written in the form

$$
\begin{aligned}
2 i \exp \left(r_{0}^{-}+i(2 \operatorname{Im} \sigma)^{-1}\right. & \left.\left(|\sigma|^{2} \log |x|-r_{0}^{-} \operatorname{Re} \sigma+\mu_{-} \operatorname{Im} \sigma\right)\right) \\
& \times \sin \left((2 \operatorname{Im} \sigma)^{-1}\left(|\sigma|^{2} \log |x|-r_{0}^{-} \operatorname{Re} \sigma-\mu_{-} \operatorname{Im} \sigma\right)\right) .
\end{aligned}
$$

This implies that $x^{\sigma}-\xi_{0}^{-}$admits a sequence $\left\{x_{\nu}\right\}_{\nu \in \mathbb{N}} \subset \Gamma\left(r_{0}^{-}, 1\right)$ of simple zeros given by

$$
|\sigma|^{2} \log \left|x_{\nu}\right|-r_{0}^{-} \operatorname{Re} \sigma-\mu_{-} \operatorname{Im} \sigma=-2 \pi \nu|\operatorname{Im} \sigma| .
$$

It is easy to see that $\left|x_{\nu+1}\right|=\exp \left(-2 \pi|\sigma|^{-2}|\operatorname{Im} \sigma|\right)\left|x_{\nu}\right|$. By the fact mentioned above, $\left|\rho^{-1} x^{1+\sigma} R_{*}(x)\right| \leq M_{0}\left|x_{\nu}\right|$ if $\left|x-x_{\nu}\right|<\left|x_{\nu}\right| / 2$, where $M_{0}$ is a positive 
number independent of $\nu$. Let $D_{\nu}(M)$ be the disc $\left|x-x_{\nu}\right| \leq M\left|x_{\nu}\right|^{2}$, where the constant $M$ independent of $\nu$ will be fixed later. On $D_{\nu}(M)$,

$$
\begin{aligned}
x^{\sigma}-\xi_{0}^{-} & =\sigma x_{\nu}^{\sigma-1}\left(x-x_{\nu}\right)\left(1+O\left(M x_{\nu}\right)\right), \\
x^{\sigma}-\xi_{0}^{+} & =\xi_{0}^{-}-\xi_{0}^{+}+\sigma x_{\nu}^{\sigma-1}\left(x-x_{\nu}\right)\left(1+O\left(M x_{\nu}\right)\right) \\
& =\left(\xi_{0}^{-}-\xi_{0}^{+}\right)\left(1+O\left(M x_{\nu}\right)\right)
\end{aligned}
$$

as long as $M\left|x_{\nu}\right|<1 / 2$. Hence, on $D_{\nu}(M), R_{1}\left(\sigma, \rho x^{\sigma}\right)$ admits no zeros other than $x_{\nu}$ if $\left|x_{\nu}\right|$ is sufficiently small. Furthermore, on the circle $\partial D_{\nu}(M)$,

$$
\left|\left(x^{\sigma}-\xi_{0}^{-}\right)\left(x^{\sigma}-\xi_{0}^{+}\right)\right|=M|\sigma| \exp \left(r_{0}^{-}\right)\left|\xi_{0}^{-}-\xi_{0}^{+}\right|\left|x_{\nu}\right|\left(1+O\left(M x_{\nu}\right)\right) .
$$

Take $M$ such that $M|\sigma| \exp \left(r_{0}^{-}\right)\left|\xi_{0}^{-}-\xi_{0}^{+}\right|=2 M_{0}$. Then $\left|\left(x^{\sigma}-\xi_{0}^{-}\right)\left(x^{\sigma}-\xi_{0}^{+}\right)\right|>$ $\left|\rho^{-1} x^{1+\sigma} R_{*}(x)\right|$ on $\partial D_{\nu}(M)$, provided that $\left|x_{\nu}\right|$ is sufficiently small, since we have $\left|x-x_{\nu}\right|<\left|x_{\nu}\right| / 2$ on $D_{\nu}(M)$ if $M\left|x_{\nu}\right|<1 / 2$. By Rouché's theorem there exists only one simple zero $x_{\nu}^{-}$of $y(\sigma, \rho, x)$ in the interior of $\partial D_{\nu}(M)$, which satisfies $\left|x_{\nu}^{-}-x_{\nu}\right|=O\left(\left|x_{\nu}\right|^{2}\right)$ if $\left|x_{\nu}\right|$ is sufficiently small. Thus we have

Proposition 2.7. If $\theta_{0} \neq 0$ and $\xi_{0}^{ \pm} \neq 0$, then $y(\sigma, \rho, x)$ admits a sequence $\left\{x_{\nu}^{-}\right\}_{\nu \in \mathbb{N}}$ of simple zeros such that

$$
|\sigma|^{2} \log \left|x_{\nu}^{-}\right|-r_{0}^{-} \operatorname{Re} \sigma-\mu_{-} \operatorname{Im} \sigma \sim-2 \pi \nu|\operatorname{Im} \sigma|
$$

and $\operatorname{dist}\left(x_{\nu}^{-}, \Gamma\left(r_{0}^{-}, 1\right)\right)=O\left(\left|x_{\nu}^{-}\right|^{2}\right)$.

Similarly, for $\left(r_{0}^{+}, \mu_{+}\right)=\left(r_{0}^{+}(\sigma, \rho), \mu_{+}(\sigma, \rho)\right)$ satisfying $\exp \left(r_{0}^{+}\right)=\left|\xi_{0}^{+}\right|, \mu_{+}=$ $\arg \xi_{0}^{+}$, we have

Proposition 2.8. If $\theta_{0} \neq 0$ and $\xi_{0}^{ \pm} \neq 0$, then $y(\sigma, \rho, x)$ admits a sequence $\left\{x_{\nu}^{+}\right\}_{\nu \in \mathbb{N}}$ of simple zeros, different from $\left\{x_{\nu}^{-}\right\}_{\nu \in \mathbb{N}}$, such that

$$
|\sigma|^{2} \log \left|x_{\nu}^{+}\right|-r_{0}^{+} \operatorname{Re} \sigma-\mu_{+} \operatorname{Im} \sigma \sim-2 \pi \nu|\operatorname{Im} \sigma|
$$

and $\operatorname{dist}\left(x_{\nu}^{+}, \Gamma\left(r_{0}^{+}, 1\right)\right)=O\left(\left|x_{\nu}^{+}\right|^{2}\right)$

Using (2.5) we may obtain inverse oscillatory solutions admitting sequences of poles (cf. Section 2.2.4).

Remark 2.9. In the plane $\mathbb{R}^{2} \ni(\log |x|, \arg x)$, the lines representing $\Gamma\left(r_{0}^{-}, 1\right)$ and $\Gamma\left(r_{0}^{+}, 1\right)$ run parallel to each other. Hence, if $\operatorname{Re} \sigma \neq 0$, the sequences $\left\{x_{\nu}^{-}\right\}_{\nu \in \mathbb{N}}$ and $\left\{x_{\nu}^{+}\right\}_{\nu \in \mathbb{N}}$ lie asymptotically along the respective spiral curves that are congruent. If $\operatorname{Re} \sigma=0$, they accumulate at $x=0$ asymptotically along the respective rays, and the corresponding pole sequences were studied in [10]. 
Double zeros. If $\theta_{0}=0$ and if $\xi_{0}^{0}=\xi_{0}^{0}(\sigma, \rho)=\left(4 \sigma^{2} \rho\right)^{-1}\left(\theta_{x}^{2}-\sigma^{2}\right) \neq 0$, then $R_{1}\left(\sigma, \rho x^{\sigma}\right)=\rho x^{-\sigma}\left(x^{\sigma}-\xi_{0}^{0}\right)^{2}$ vanishes at $x_{\nu} \in \Gamma\left(r_{0}^{0}, 1\right)$ such that

$$
|\sigma|^{2} \log \left|x_{\nu}\right|-r_{0}^{0} \operatorname{Re} \sigma-\mu_{0} \operatorname{Im} \sigma=-2 \pi \nu|\operatorname{Im} \sigma|,
$$

which is a double zero, where $r_{0}^{0}=r_{0}^{0}(\sigma, \rho)$ and $\mu_{0}=\mu_{0}(\sigma, \rho)$ are given by $\exp \left(r_{0}^{0}\right)=\left|\xi_{0}^{0}\right|, \mu_{0}=\arg \xi_{0}^{0}$. Every zero of solutions of (PVI) is double if $\theta_{0}=0$, which is checked by a straightforward computation. Using this fact, by the same argument as above, we have

Proposition 2.9. If $\theta_{0}=0$ and $\xi_{0}^{0} \neq 0$, then $y(\sigma, \rho, x)$ admits a sequence $\left\{x_{\nu}^{0}\right\}_{\nu \in \mathbb{N}}$ of double zeros such that

$$
|\sigma|^{2} \log \left|x_{\nu}^{0}\right|-r_{0}^{0} \operatorname{Re} \sigma-\mu_{0} \operatorname{Im} \sigma \sim-2 \pi \nu|\operatorname{Im} \sigma|
$$

and $\operatorname{dist}\left(x_{\nu}^{0}, \Gamma\left(r_{0}^{0}, 1\right)\right)=O\left(\left|x_{\nu}^{0}\right|^{2}\right)$.

2.2.3. Non-decaying exponential oscillation. Suppose that $\omega=0$. In this case $y(\sigma, \rho, x)$ along $\Gamma\left(r_{0}, 0\right)$ is not asymptotic to $x R_{1}\left(\sigma, \rho x^{\sigma}\right)$. Let $r_{0} \in \mathbb{R}$ be a given number, and let $\rho$ be such that $\left|\rho x^{1+\sigma}\right|=|\rho| e^{r_{0}}<\hat{\varepsilon}_{0}$. Then

$$
x^{1+\sigma}=e^{r_{0}} \exp \left(i(\operatorname{Im} \sigma)^{-1}\left(\left(|\sigma|^{2}+2 \operatorname{Re} \sigma+1\right) \log |x|-r_{0}(1+\operatorname{Re} \sigma)\right)\right)
$$

along $\Gamma\left(r_{0}, 0\right)$. Since

$$
x^{n} R_{n}\left(\sigma, \rho x^{\sigma}\right)=\sum_{j=0}^{2 n} c_{n-j}^{n}(\sigma) \rho^{n-j}\left(x^{1+\sigma}\right)^{n-j} x^{j}=c_{n}^{n}(\sigma) \rho^{n}\left(x^{1+\sigma}\right)^{n}+O(x),
$$

$y(\sigma, \rho, x)$ is asymptotic to the sum of non-decaying exponential oscillatory terms.

Proposition 2.10. If $|\rho| e^{r_{0}}=\left|\rho x^{1+\sigma}\right|$ is sufficiently small, then, along $\Gamma\left(r_{0}, 0\right)$,

$$
y(\sigma, \rho, x)=\sum_{n=1}^{\infty} c_{n}^{n}(\sigma)\left(\rho e^{r_{0}}\right)^{n} \eta\left(\sigma, r_{0}, x\right)^{n}+O(x)
$$

with

$$
\eta\left(\sigma, r_{0}, x\right)=\exp \left(i(\operatorname{Im} \sigma)^{-1}\left(\left(|\sigma|^{2}+2 \operatorname{Re} \sigma+1\right) \log |x|-r_{0}(1+\operatorname{Re} \sigma)\right)\right) .
$$

Along $\Gamma\left(r_{0}, 2\right)$ we have a solution of the same type.

2.2.4. Analytic continuation around $x=0$ and inverse oscillation. Let $\sigma$ and $\rho$ be generic values. By matching we discuss the analytic continuation of 
$y(\sigma, \rho, x)$ beyond the region $\bigcup_{0<\omega<2} \Gamma\left(r_{0}, \omega\right)$. Denote $\Gamma\left(r_{0}, \omega\right)$ by $\Gamma\left(r_{0}, \omega\right)_{\sigma}$, indicating the dependence on $\sigma$. By Proposition 2.6, along $\Gamma\left(r_{0}, \omega\right)_{\sigma}$,

$$
\begin{array}{ll}
y(\sigma, \rho, x) \sim \rho x^{1+\sigma} & \text { if } 0<\omega<1, \\
y(\sigma, \rho, x) \sim c_{-1}^{1}(\sigma) \rho^{-1} x^{1-\sigma} & \text { if } 1<\omega<2 \text { and } c_{-1}^{1}(\sigma) \neq 0 .
\end{array}
$$

To $y(\sigma, \rho, x)$ and $c_{-1}^{1}(\sigma)$ we apply the substitution

$$
\left(\theta_{0}, \theta_{x}, \theta_{1}, \theta_{\infty}\right) \mapsto\left(\theta_{\infty}-1, \theta_{1}, \theta_{x}, \theta_{0}+1\right),
$$

and denote the results by $\tilde{y}(\sigma, \rho, x)$ and $\tilde{c}_{-1}^{1}(\sigma)$, respectively. Then $y_{\infty}(\tilde{\sigma}, \tilde{\rho}, x):=$ $x / \tilde{y}(\tilde{\sigma}, \tilde{\rho}, x)$ also solves (PVI) (cf. (2.5)), and along $\Gamma\left(r_{0}, \tilde{\omega}\right) \tilde{\sigma}$,

$$
\begin{array}{lll}
y_{\infty}(\tilde{\sigma}, \tilde{\rho}, x) \sim \tilde{\rho}^{-1} x^{-\tilde{\sigma}} & & \text { if } 0<\tilde{\omega}<1, \\
y_{\infty}(\tilde{\sigma}, \tilde{\rho}, x) \sim \tilde{c}_{-1}^{1}(\tilde{\sigma})^{-1} \tilde{\rho} x^{\tilde{\sigma}} & & \text { if } 1<\tilde{\omega}<2 \text { and } \tilde{c}_{-1}^{1}(\tilde{\sigma}) \neq 0 .
\end{array}
$$

For this solution we observe inverse oscillatory behaviours derived from Propositions 2.7 through 2.9 .

Proposition 2.11. (1) Suppose that $\theta_{\infty} \neq 1$ and $\tilde{c}_{-1}^{1}(\tilde{\sigma}) \neq 0$. Then $y_{\infty}(\tilde{\sigma}, \tilde{\rho}, x)$ admits sequences $\left\{x_{\nu}^{\infty-}\right\}_{\nu \in \mathbb{N}}$ and $\left\{x_{\nu}^{\infty+}\right\}_{\nu \in \mathbb{N}}$ of simple poles such that

$$
|\tilde{\sigma}|^{2} \log \left|x_{\nu}^{\infty \pm}\right|-r_{0}^{\infty \pm} \operatorname{Re} \tilde{\sigma}-\mu_{ \pm}^{\infty} \operatorname{Im} \tilde{\sigma} \sim-2 \pi \nu|\operatorname{Im} \tilde{\sigma}|
$$

and $\operatorname{dist}\left(x_{\nu}^{\infty \pm}, \Gamma\left(r_{0}^{\infty \pm}, 1\right)_{\tilde{\sigma}}\right)=O\left(\left|x_{\nu}^{\infty \pm}\right|^{2}\right)$, where $r_{0}^{\infty \pm}=r_{0}^{\infty \pm}(\tilde{\sigma}, \tilde{\rho})$ and $\mu_{ \pm}^{\infty}=$ $\mu_{ \pm}^{\infty}(\tilde{\sigma}, \tilde{\rho})$ are given by

$$
\begin{aligned}
\exp \left(r_{0}^{\infty \pm}\right) & =\left|\left(4 \tilde{\sigma}^{2} \tilde{\rho}\right)^{-1}\left(\theta_{1}^{2}-\left(\theta_{\infty}-1 \pm \tilde{\sigma}\right)^{2}\right)\right| \\
\mu_{ \pm}^{\infty} & =\arg \left(\left(4 \tilde{\sigma}^{2} \tilde{\rho}\right)^{-1}\left(\theta_{1}^{2}-\left(\theta_{\infty}-1 \pm \tilde{\sigma}\right)^{2}\right)\right) .
\end{aligned}
$$

(2) Suppose that $\theta_{\infty}=1$ and $\tilde{c}_{-1}^{1}(\tilde{\sigma}) \neq 0$. Then $y_{\infty}(\tilde{\sigma}, \tilde{\rho}, x)$ admits a sequence $\left\{x_{\nu}^{\infty 0}\right\}_{\nu \in \mathbb{N}}$ of double poles such that

$$
|\tilde{\sigma}|^{2} \log \left|x_{\nu}^{\infty 0}\right|-r_{0}^{\infty 0} \operatorname{Re} \tilde{\sigma}-\mu_{0}^{\infty} \operatorname{Im} \tilde{\sigma} \sim-2 \pi \nu|\operatorname{Im} \tilde{\sigma}|
$$

and $\operatorname{dist}\left(x_{\nu}^{\infty 0}, \Gamma\left(r_{0}^{\infty 0}, 1\right)_{\tilde{\sigma}}\right)=O\left(\left|x_{\nu}^{\infty 0}\right|^{2}\right)$, where $r_{0}^{\infty 0}=r_{0}^{\infty 0}(\tilde{\sigma}, \tilde{\rho})$ and $\mu_{0}^{\infty}=\mu_{0}^{\infty}(\tilde{\sigma}, \tilde{\rho})$ are given by

$$
\exp \left(r_{0}^{\infty 0}\right)=\left|\left(4 \tilde{\sigma}^{2} \tilde{\rho}\right)^{-1}\left(\theta_{1}^{2}-\tilde{\sigma}^{2}\right)\right|, \quad \mu_{0}^{\infty}=\arg \left(\left(4 \tilde{\sigma}^{2} \tilde{\rho}\right)^{-1}\left(\theta_{1}^{2}-\tilde{\sigma}^{2}\right)\right) .
$$

By (2.6) and Remark 2.4, say along $\Gamma\left(r_{0}, \omega\right)_{\sigma}$ with $0<\omega<1$,

$$
y(\sigma, \rho, x)=\rho x^{1+\sigma}(1+o(1)), \quad x y^{\prime}(\sigma, \rho, x)=\rho x^{1+\sigma}(1+\sigma+o(1)) .
$$

The uniqueness of a solution behaving like this is verified by using an equation describing (PVI) locally (see Appendix). Let us consider matching (2.6) with (2.9). 
By the definition of $\Gamma\left(r_{0}, \omega\right)_{\sigma}$, we have the relation $\Gamma\left(r_{0}, \omega+1\right)_{\sigma+1}=\Gamma\left(r_{0}, \omega\right)_{\sigma}$. Observing that $y_{\infty}(1+\sigma, \tilde{\rho}, x) \sim \tilde{c}_{-1}^{1}(\sigma+1)^{-1} \tilde{\rho} x^{1+\sigma}$ along $\Gamma\left(r_{0}, 1+\omega\right)_{1+\sigma}$ if $0<\omega<1$, we have $y(\sigma, \rho, x)=y_{\infty}(1+\sigma, \tilde{\rho}, x)$ if $\tilde{c}_{-1}^{1}(\sigma+1)^{-1} \tilde{\rho}=\rho$. Since both sides are analytic in $(\sigma, \rho, x)$, we have

$$
y(\sigma, \rho, x) \equiv y_{\infty}\left(1+\sigma, \tilde{c}_{-1}^{1}(\sigma+1) \rho, x\right) .
$$

More generally, for every $\nu \in \mathbb{Z}$, observing that

$$
\Gamma\left(r_{0}, \omega\right)_{\sigma}=\Gamma\left(r_{0}, \omega-2 \nu\right)_{\sigma-2 \nu}=\Gamma\left(r_{0}, \omega-2 \nu+1\right)_{\sigma-2 \nu+1},
$$

from (2.6) through (2.9) we derive the following:

Proposition 2.12. For every $\nu \in \mathbb{Z}$ and every $r_{0} \in \mathbb{R}$, along $\Gamma\left(r_{0}, \omega\right)_{\sigma}$,

$$
y(\sigma-2 \nu, \rho, x) \sim \begin{cases}\rho x^{1-2 \nu+\sigma} & \text { if } 2 \nu<\omega<2 \nu+1, \\ c_{-1}^{1}(\sigma-2 \nu) \rho^{-1} x^{1+2 \nu-\sigma} & \text { if } 2 \nu+1<\omega<2 \nu+2\end{cases}
$$

as long as $c_{-1}^{1}(\sigma-2 \nu) \neq 0$, and

$$
y_{\infty}(\sigma-2 \nu+1, \tilde{\rho}, x) \sim \begin{cases}\tilde{\rho}^{-1} x^{2 \nu-1-\sigma} & \text { if } 2 \nu-1<\omega<2 \nu \\ \tilde{c}_{-1}^{1}(\sigma-2 \nu+1)^{-1} \tilde{\rho} x^{-2 \nu+1+\sigma} & \text { if } 2 \nu<\omega<2 \nu+1\end{cases}
$$

as long as $\tilde{c}_{-1}^{1}(\sigma-2 \nu+1) \neq 0$.

Then by matching we obtain the following relations, which combined with Proposition 2.12 enable us to find the analytic continuation of $y(\sigma, \rho, x)$ or $y_{\infty}(\sigma, \rho, x)$ around $x=0$.

Proposition 2.13. For any $\nu \in \mathbb{Z}$,

$$
\begin{aligned}
& y(\sigma-2 \nu, \rho, x) \equiv y_{\infty}\left(\sigma-2 \nu+1, \tilde{c}_{-1}^{1}(\sigma-2 \nu+1) \rho, x\right), \\
& y\left(\sigma-2 \nu, c_{-1}^{1}(\sigma-2 \nu) \rho, x\right) \equiv y_{\infty}(\sigma-2 \nu-1, \rho, x), \\
& y(\sigma-2 \nu, \rho, x) \equiv y\left(\sigma-2 \nu+2, \tilde{c}_{-1}^{1}(\sigma-2 \nu+1) c_{-1}^{1}(\sigma-2 \nu+2) \rho, x\right), \\
& y_{\infty}\left(\sigma-2 \nu+1, c_{-1}^{1}(\sigma-2 \nu) \tilde{c}_{-1}^{1}(\sigma-2 \nu+1) \rho, x\right) \equiv y_{\infty}(\sigma-2 \nu-1, \rho, x)
\end{aligned}
$$

as long as $c_{-1}^{1}(\cdots), \tilde{c}_{-1}^{1}(\cdots)$ in each relation do not vanish.

For $y(\sigma, \rho, x)$ along $\Gamma\left(r_{0}, 0\right)_{\sigma}$ or $\Gamma\left(r_{0}, 2\right)_{\sigma}$, a non-decaying exponential oscillation is observed as in Proposition 2.10, provided that $\rho e^{r_{0}}$ or $\left(\rho e^{r_{0}}\right)^{-1}$ is sufficiently small, that is, $\Gamma\left(r_{0}, 0\right)_{\sigma}$ or $\Gamma\left(r_{0}, 2\right)_{\sigma}$ enters $\Omega_{\sigma, \rho}\left(\hat{\varepsilon}_{0}\right)$. By the relations above with $\nu=0$, sequences of poles appear asymptotically along $\Gamma\left(r_{0}^{\sharp}, 0\right)_{\sigma}=\Gamma\left(r_{0}^{\sharp}, 1\right)_{\sigma+1}$ or $\Gamma\left(r_{0}^{\sharp}, 2\right)_{\sigma}=\Gamma\left(r_{0}^{\sharp}, 1\right)_{\sigma-1}$, where $r_{0}^{\sharp} \in\left\{r_{0}^{\infty-}, r_{0}^{\infty+}, r_{0}^{\infty 0}\right\}$ is given in Proposition 2.11 
with $\tilde{\sigma}=\sigma+1$ or $\sigma-1$, on which the condition for the non-decaying exponential oscillation is violated. Further pole sequences as well as zero sequences are obtained from Proposition 2.13 as long as $c_{-1}^{1}(\sigma+\nu), \tilde{c}_{-1}^{1}(\sigma+\nu) \neq 0$. These pole sequences are situated as conjectured in [4], [5].

2.2.5. On $y_{+}\left(\sigma_{0}, \rho, x\right)$. The solution $y_{+}\left(\sigma_{0}, \rho, x\right)$ may also be observed along

$$
\Gamma_{0}\left(r_{0}, \omega\right): \quad\left(1+\operatorname{Re} \sigma_{0}-\omega\right) \log |x|-\operatorname{Im} \sigma_{0} \cdot \arg x=r_{0}
$$

if $\operatorname{Im} \sigma_{0} \neq 0$, on which $\left|x^{1+\sigma_{0}}\right|=e^{r_{0}}|x|^{\omega}$, where $r_{0} \in \mathbb{R}, \omega \geq 0$. Suppose that $\sigma_{0}^{2}+\theta_{0}^{2}-\theta_{x}^{2} \neq 0$. For every $r_{0} \in \mathbb{R}$, along $\Gamma_{0}\left(r_{0}, \omega\right)$,

$$
y_{+}\left(\sigma_{0}, \rho, x\right) \sim \begin{cases}\rho x^{1+\sigma_{0}} & \text { if } 0<\omega<1 \\ \left(2 \sigma_{0}^{2}\right)^{-1}\left(\sigma_{0}^{2}+\theta_{0}^{2}-\theta_{x}^{2}\right) x & \text { if } \omega>1\end{cases}
$$

Suppose that $\omega=1$. Let $r_{0}^{*}=r_{0}^{*}\left(\sigma_{0}, \rho\right)$ and $\mu_{*}=\mu_{*}\left(\sigma_{0}, \rho\right)$ be such that

$$
\exp \left(r_{0}^{*}\right)=\left|-\left(2 \sigma_{0}^{2} \rho\right)^{-1}\left(\sigma_{0}^{2}+\theta_{0}^{2}-\theta_{x}^{2}\right)\right|, \quad \mu_{*}=\arg \left(-\left(2 \sigma_{0}^{2} \rho\right)^{-1}\left(\sigma_{0}^{2}+\theta_{0}^{2}-\theta_{x}^{2}\right)\right) .
$$

Then $y_{+}\left(\sigma_{0}, \rho, x\right)$ admits a sequence $\left\{x_{\nu}^{*}\right\}_{\nu \in \mathbb{N}}$ of simple zeros such that

$$
\left|\sigma_{0}\right|^{2} \log \left|x_{\nu}^{*}\right|-r_{0}^{*} \operatorname{Re} \sigma_{0}-\mu_{*} \operatorname{Im} \sigma_{0} \sim-2 \pi \nu\left|\operatorname{Im} \sigma_{0}\right|
$$

and $\operatorname{dist}\left(x_{\nu}^{*}, \Gamma_{0}\left(r_{0}^{*}, 1\right)\right)=O\left(\left|x_{\nu}^{*}\right|^{2}\right)$. If $\omega=0$, for $\rho$ such that $\left|\rho x^{1+\sigma_{0}}\right|=|\rho| e^{r_{0}}<\varepsilon_{0}^{+}$,

$$
y_{+}\left(\sigma_{0}, \rho, x\right)=\sum_{n=1}^{\infty} c_{n}^{n}\left(\sigma_{0}\right)\left(\rho e^{r_{0}}\right)^{n} \eta\left(\sigma_{0}, r_{0}, x\right)^{n}+O(x)
$$

along $\Gamma_{0}\left(r_{0}, 0\right)$. Note that $c_{-1}^{1}\left(\sigma_{0}\right)=0$. If $\tilde{\sigma}_{0}$ satisfies $\tilde{c}_{-1}^{1}\left(\tilde{\sigma}_{0}\right)=0, \operatorname{Im} \tilde{\sigma}_{0} \neq 0$ and $\tilde{\sigma}_{0}^{2}+\left(\theta_{\infty}-1\right)^{2}-\theta_{1}^{2} \neq 0$, where $\tilde{c}_{-1}^{1}(\sigma)$ is as in Section 2.2 .4 , then (2.5) yields an inverse oscillatory solution $y_{\infty+}\left(\tilde{\sigma}_{0}, \tilde{\rho}, x\right)$ such that, along $\Gamma_{0}\left(r_{0}, \tilde{\omega}\right)_{\tilde{\sigma}_{0}}$

$$
y_{\infty+}\left(\tilde{\sigma}_{0}, \tilde{\rho}, x\right) \sim \begin{cases}\tilde{\rho}^{-1} x^{-\tilde{\sigma}_{0}} & \text { if } 0<\tilde{\omega}<1, \\ 2 \tilde{\sigma}_{0}^{2}\left(\tilde{\sigma}_{0}^{2}+\left(\theta_{\infty}-1\right)^{2}-\theta_{1}^{2}\right)^{-1} & \text { if } \tilde{\omega}>1 .\end{cases}
$$

Furthermore it admits a sequence $\left\{x_{\nu}^{\infty *}\right\}_{\nu \in \mathbb{N}}$ of simple poles such that

$$
\left|\tilde{\sigma}_{0}\right|^{2} \log \left|x_{\nu}^{\infty *}\right|-r_{0}^{\infty *} \operatorname{Re} \tilde{\sigma}_{0}-\mu_{*}^{\infty} \operatorname{Im} \tilde{\sigma}_{0} \sim-2 \pi \nu\left|\operatorname{Im} \tilde{\sigma}_{0}\right|
$$

and $\operatorname{dist}\left(x_{\nu}^{\infty *}, \Gamma_{0}\left(r_{0}^{\infty *}, 1\right)_{\tilde{\sigma}_{0}}\right)=O\left(\left|x_{\nu}^{\infty *}\right|^{2}\right)$, where

$$
\begin{aligned}
\exp \left(r_{0}^{\infty *}\right) & =\left|-\left(2 \tilde{\sigma}_{0}^{2} \tilde{\rho}\right)^{-1}\left(\tilde{\sigma}_{0}^{2}+\left(\theta_{\infty}-1\right)^{2}-\theta_{1}^{2}\right)\right|, \\
\mu_{*}^{\infty} & =\arg \left(-\left(2 \tilde{\sigma}_{0}^{2} \tilde{\rho}\right)^{-1}\left(\tilde{\sigma}_{0}^{2}+\left(\theta_{\infty}-1\right)^{2}-\theta_{1}^{2}\right)\right) .
\end{aligned}
$$


§3. Isomonodromy deformation, the Schlesinger equation and (PVI)

If $\left(A_{0}(x), A_{x}(x), A_{1}(x)\right)$ is a solution of (2.1) such that

(i) the eigenvalues of $A_{0}(x), A_{x}(x), A_{1}(x)$ are $\pm \theta_{0} / 2, \pm \theta_{x} / 2, \pm \theta_{1} / 2$, respectively,

(ii) $A_{0}(x)+A_{x}(x)+A_{1}(x) \equiv-\left(\theta_{\infty} / 2\right) J$,

then the Fuchsian system

$$
\frac{d Y}{d \lambda}=\left(\frac{A_{0}(x)}{\lambda}+\frac{A_{x}(x)}{\lambda-x}+\frac{A_{1}(x)}{\lambda-1}\right) Y
$$

admits a fundamental matrix solution whose monodromy remains invariant under a small variation of the deformation parameter $x$, and

$$
y(x)=\frac{x A_{0}(x)_{12}}{x\left(A_{0}(x)+A_{1}(x)\right)_{12}-A_{1}(x)_{12}}
$$

solves (PVI) with $(\alpha, \beta, \gamma, \delta)$ satisfying (2.2). As guaranteed by the following proposition, our solutions of (2.1) satisfy (i) and (ii), and in solving (PVI) we rely on this fact (see Section 6).

Proposition 3.1. The solution $\left(A_{0}(x), A_{x}(x), A_{1}(x)\right)$ of (2.1) given by Theorem 2.1 or 2.2 satisfies (i) and (ii) above, and the corresponding system (3.1) has the isomonodromy property.

Proof. Suppose that $\left(A_{0}(x), A_{x}(x), A_{1}(x)\right)$ is as in, say, Theorem 2.2. By Theorem 2.2,

$$
\begin{aligned}
A_{0}(x) & +A_{x}(x)+A_{1}(x) \\
& =(\rho x)^{\Lambda}\left(\Lambda_{0}+\Lambda_{x}+O\left(\left|x \log ^{2}(\rho x)\right|\right)\right)(\rho x)^{-\Lambda}+\Lambda_{1}+O\left(\left|x \log ^{2}(\rho x)\right|\right) \\
& =\Lambda_{0}+\Lambda_{x}+\Lambda_{1}+O\left(\left|x \log ^{4}(\rho x)\right|\right)=-\left(\theta_{\infty} / 2\right) J+O\left(|x|^{1 / 2}\right)
\end{aligned}
$$

as $x \rightarrow 0$ through $\Omega_{\rho}^{*}\left(\varepsilon_{0}, \Theta_{0}\right)$. From (2.1) it follows that $(d / d x)\left(A_{0}(x)+A_{x}(x)+\right.$ $\left.A_{1}(x)\right) \equiv 0$. Hence $A_{0}(x)+A_{x}(x)+A_{1}(x) \equiv-\left(\theta_{\infty} / 2\right) J$, which is property (ii). Fix $\left(\rho, x_{0}\right) \in \Omega^{*}\left(\varepsilon_{0}, \Theta_{0}\right)$ and consider the Pfaffian system

$$
d Y=\left(A_{0}(x) \frac{d \lambda}{\lambda}+A_{x}(x) \frac{d(\lambda-x)}{\lambda-x}+A_{1}(x) \frac{d(\lambda-1)}{\lambda-1}\right) Y
$$

for $(x, \lambda) \in\left\{x \in \Omega_{\rho}^{*}\left(\varepsilon_{0}, \Theta_{0}\right) ;\left|x-x_{0}\right|<\varepsilon_{0}^{\prime}\right\} \times \mathbb{P}^{1}$, where $\varepsilon_{0}^{\prime}=\varepsilon_{0}^{\prime}\left(x_{0}, \rho\right)<\left|x_{0}\right| / 2$ is a sufficiently small positive number. System (3.2) is completely integrable since $\left(A_{0}(x), A_{x}(x), A_{1}(x)\right)$ solves (2.1). Let $Y(x, \lambda)$ be a fundamental matrix solution of (3.2) such that, for $\left|x-x_{0}\right|<\varepsilon_{0}^{\prime}$,

$$
Y(x, \lambda)=\left(I+O\left(\lambda^{-1}\right)\right) \lambda^{-\left(\theta_{\infty} / 2\right) J} \lambda^{\Delta_{\infty}} \quad \text { around } \lambda=\infty
$$


with $\Delta_{\infty}$ denoting $\epsilon_{\infty} \Delta$ if $\theta_{\infty} \in \mathbb{N}, \epsilon_{\infty} \Delta_{-}$if $-\theta_{\infty} \in \mathbb{N}$, and 0 if $\theta_{\infty} \notin \mathbb{Z}$, where $\epsilon_{\infty} \in \mathbb{C}$ (cf. [18]). As $(d / d x) \operatorname{tr} A_{\iota}(x) \equiv 0$ and $\operatorname{tr} A_{\iota}(x)=O\left(\left|x \log ^{4}(\rho x)\right|\right)$, we have $\operatorname{tr} A_{\iota}(x) \equiv 0(\iota=0, x, 1)$. Let $\pm \theta_{\iota}^{0} / 2$ be the eigenvalues of $A_{\iota}\left(x_{0}\right)$. Then, by [18], $Y(x, \lambda)$ behaves as follows:

$$
\begin{array}{ll}
G_{0}(x)(I+O(\lambda)) \lambda^{\left(\theta_{0}^{0} / 2\right) J} \lambda^{\Delta_{0}} \Gamma_{0} & \text { around } \lambda=0, \\
G_{x}(x)(I+O(\lambda-x))(\lambda-x)^{\left(\theta_{x}^{0} / 2\right) J}(\lambda-x)^{\Delta_{x}} \Gamma_{x} & \text { around } \lambda=x, \\
G_{1}(x)(I+O(\lambda-1))(\lambda-1)^{\left(\theta_{1}^{0} / 2\right) J}(\lambda-1)^{\Delta_{1}} \Gamma_{1} & \text { around } \lambda=1
\end{array}
$$

for $\left|x-x_{0}\right|<\varepsilon_{0}^{\prime}$ with connection matrices $\Gamma_{0}, \Gamma_{x}, \Gamma_{1}$ independent of $x$. Here, for each $\iota \in\{0, x, 1\}, G_{\iota}(x)$ is an invertible matrix holomorphic for $\left|x-x_{0}\right|<\varepsilon_{0}^{\prime}$, and $\Delta_{\iota}$ denotes $\epsilon_{\iota} \Delta$ if $\theta_{\iota}^{0} \in \mathbb{N} \cup\{0\}, \epsilon_{\iota} \Delta_{-}$if $-\theta_{\iota}^{0} \in \mathbb{N}$, and 0 if $\theta_{\iota}^{0} \notin \mathbb{Z}$, where $\epsilon_{\iota} \in \mathbb{C}$. This fact implies that the monodromy with respect to $Y(x, \lambda)$ as a solution of (3.1) does not depend on the deformation parameter $x$. The local behaviour around $\lambda=0$ gives

$$
\lambda^{-1} A_{0}(x)+O(1)=\frac{\partial Y}{\partial \lambda}(x, \lambda) Y(x, \lambda)^{-1}=\lambda^{-1} G_{0}(x) V(\lambda) G_{0}(x)^{-1}
$$

with

$$
V(\lambda)=(I+O(\lambda))\left(\left(\theta_{0}^{0} / 2\right) J+\lambda^{\left(\theta_{0}^{0} / 2\right) J} \Delta_{0} \lambda^{-\left(\theta_{0}^{0} / 2\right) J}\right)(I+O(\lambda))+O(\lambda),
$$

whose residue is

$$
A_{0}(x)=G_{0}(x)\left(\theta_{0}^{0} / 2\right) J G_{0}(x)^{-1} \quad \text { or } \quad G_{0}(x)\left(\left(\theta_{0}^{0} / 2\right) J+\Delta_{0}\right) G_{0}(x)^{-1},
$$

which implies that the eigenvalues of $A_{0}(x)$ are $\pm \theta_{0}^{0} / 2$. Combining this fact with $(\rho x)^{-\Lambda} A_{0}(x)(\rho x)^{\Lambda}=\Lambda_{0}+O\left(\left|x \log ^{2}(\rho x)\right|\right)$, we find $\theta_{0}^{0}=\theta_{0}$. Similarly we show that the eigenvalues of $A_{x}(x)$ and $A_{1}(x)$ are $\pm \theta_{x} / 2$ and $\pm \theta_{1} / 2$, respectively, which completes the proof.

\section{$\S 4$. Rings of matrix series}

\section{§4.1. Logarithmic type}

Let $\widehat{\mathfrak{L}}$ be the ring of formal series of the form

$$
\Phi=\Phi(\kappa, t)=\sum_{n=1}^{\infty} \sum_{m=0}^{2 n} C_{m}^{n}(\kappa t)^{n} \log ^{m} t, \quad C_{m}^{n} \in M_{2}\left(\mathbb{C}_{\theta}\right),
$$

with the parameter $\kappa \neq 0$. For $\Phi \in \widehat{\mathfrak{L}}$ as above define the norm of $\Phi$ by

$$
\|\Phi\|=\|\Phi\|(\kappa, t):=\sum_{n=1}^{\infty} \sum_{m=0}^{2 n}\left\|C_{m}^{n}\right\||\kappa t|^{n}|t|^{-m / 4},
$$


where $\left\|C_{m}^{n}\right\|$ denotes the standard norm of the matrix $C_{m}^{n}$ such that $\|C\|=$ $\|C\|_{\text {mat }}=\max _{i=1,2}\left\{\left|C_{i 1}\right|+\left|C_{i 2}\right|\right\}$. Then $\left\|C(\kappa t)^{n} \log ^{m} t\right\|_{\text {mat }}=\|C\|_{\text {mat }}|\kappa t|^{n}|\log t|^{m}$ $\leq\|C\|_{\text {mat }}|\kappa t|^{n}|t|^{-m / 4}=\left\|C(\kappa t)^{n} \log ^{m} t\right\|$ if $|\log t| \leq|t|^{-1 / 4}$. For simplicity we use the common symbol $\|\cdot\|$ provided it causes no confusion. Let $D \subset(\mathbb{C} \backslash\{0\}) \times \mathcal{R}(\mathbb{C} \backslash\{0\})$ be a domain such that $|\log t| \leq|t|^{-1 / 4}$ for every $(\kappa, t) \in D$, and let

$$
\mathfrak{L}(D):=\left\{\Phi \in \widehat{\mathfrak{L}} ;\|\Phi\|<\infty \text { for }(\kappa, t) \in D \text { and for }\left(\theta_{\iota}\right) \text { satisfying }(2.4)\right\} .
$$

Throughout this section we suppose that $\left(\theta_{\iota}\right)$ satisfies (2.4).

Proposition 4.1. (1) If $\Phi \in \mathfrak{L}(D)$, then, for each $\left(\theta_{\iota}\right), \Phi=\Phi(\kappa, t)$ is holomorphic in $(\kappa, t) \in D$, and satisfies $\|\Phi(\kappa, t)\|_{\text {mat }} \leq\|\Phi\|(\kappa, t)$.

(2) $\|\Phi\| \equiv 0$ if and only if $\Phi \equiv 0$.

(3) Let $\Phi, \Psi \in \mathfrak{L}(D)$. Then $\Phi+\Psi, \Phi \Psi \in \mathfrak{L}(D)$, and

$$
\|\Phi+\Psi\| \leq\|\Phi\|+\|\Psi\|, \quad\|\Phi \Psi\| \leq\|\Phi\|\|\Psi\| .
$$

If $C \in M_{2}\left(\mathbb{C}_{\theta}\right)$ (respectively, $\left.c \in \mathbb{C}_{\theta}\right)$, then $C \Phi, \Phi C \in \mathfrak{L}(D)$ (respectively, $c \Phi \in \mathfrak{L}(D))$, and

$$
\|C \Phi\|,\|\Phi C\| \leq\|C\|\|\Phi\| \quad \text { (respectively, }\|c \Phi\|=|c|\|\Phi\|) .
$$

(4) Let $\Phi \in \mathfrak{L}(D)$, and let $\varphi(\tau)=\sum_{n=0}^{\infty} \varphi_{n} \tau^{n}$ with $\varphi_{n} \in \mathbb{C}$. If $|\varphi|(|\kappa t|):=$ $\sum_{n=0}^{\infty}\left|\varphi_{n}\right||\kappa t|^{n}<\infty$ in $D$, then $\varphi(\kappa t) \Phi \in \mathfrak{L}(D)$ and $\|\varphi(\kappa t) \Phi\| \leq|\varphi|(|\kappa t|)\|\Phi\|$.

Proof. Suppose that $\Phi(\kappa, t)=\sum_{n=1}^{\infty} \sum_{m=0}^{2 n} C_{m}^{n}(\kappa t)^{n} \log ^{m} t \in \mathfrak{L}(D)$. For any $(\tilde{\kappa}, \tilde{t})$ in $D$, we may choose a neighbourhood $D_{0} \subset D$ of $(\tilde{\kappa}, \tilde{t})$, a point $\left(\kappa_{0}, t_{0}\right) \in D \backslash D_{0}$, and a positive number $\varepsilon_{*}$ in such a way that $|\kappa t| \leq\left(1-\varepsilon_{*}\right)\left|\kappa_{0} t_{0}\right|,|t| \leq\left(1-\varepsilon_{*}\right)\left|t_{0}\right|$ for every $(\kappa, t) \in D_{0}$. Since $\|\Phi\|\left(\kappa_{0}, t_{0}\right)<\infty$, we have $\left\|C_{m}^{n}\right\|=O\left(\left|\kappa_{0} t_{0}\right|^{-n}\left|t_{0}\right|^{m / 4}\right)$, the implied constant being independent of $n$ and $m$. Hence

$$
\begin{aligned}
\left\|C_{m}^{n}(\kappa t)^{n} \log ^{m} t\right\|_{\text {mat }} & \leq\left\|C_{m}^{n}\right\||\kappa t|^{n}|t|^{-m / 4}=O\left(\left(|\kappa t| /\left|\kappa_{0} t_{0}\right|\right)^{n}\left(|t| /\left|t_{0}\right|\right)^{-m / 4}\right) \\
& =O\left(\left(1-\varepsilon_{*}\right)^{n-m / 4}\right)=O\left(\left(1-\varepsilon_{*}\right)^{n / 2}\right)
\end{aligned}
$$

in $D_{0}$, which implies the holomorphic nature and the inequality in (1). The remaining assertions are easily checked.

Proposition 4.2. Suppose that each $\Phi_{\nu} \in \mathfrak{L}(D)$ consists of summands with $n \geq \nu$, and that, for each $\left(\theta_{\iota}\right), \sum_{\nu=1}^{\infty}\left\|\Phi_{\nu}\right\|<\infty$ in D. Then $\Phi^{\infty}=\sum_{\nu=1}^{\infty} \Phi_{\nu} \in \mathfrak{L}(D)$, and $\left\|\Phi^{\infty}\right\| \leq \sum_{\nu=1}^{\infty}\left\|\Phi_{\nu}\right\|$.

Proof. Write $\Phi_{\nu}=\sum_{n=\nu}^{\infty} \sum_{m=0}^{2 n} C_{\nu m}^{n}(\kappa t)^{n} \log ^{m} t$ with $C_{\nu m}^{n} \in M_{2}\left(\mathbb{C}_{\theta}\right)$. Then each entry of the triple series $\sum_{\nu=1}^{\infty} \sum_{n=\nu}^{\infty} \sum_{m=0}^{2 n} C_{\nu m}^{n}(\kappa t)^{n} \log ^{m} t$ converges absolutely 
in $D$, and hence it is possible to rearrange the summands. Thus we have

$$
\Phi^{\infty}=\sum_{n=1}^{\infty} \sum_{m=0}^{2 n} C_{m}^{\infty n}(\kappa t)^{n} \log ^{m} t, \quad C_{m}^{\infty n}:=\sum_{\nu=1}^{n} C_{\nu m}^{n} \in M_{2}\left(\mathbb{C}_{\theta}\right)
$$

which satisfies $\left\|\Phi^{\infty}\right\| \leq \sum_{\nu=1}^{\infty}\left\|\Phi_{\nu}\right\|<\infty$ in $D$ and $\Phi^{\infty} \in \mathfrak{L}(D)$. This completes the proof.

Note that the primitive function of $t^{-1}(\kappa t)^{n} \log ^{m} t$ is

$$
\frac{(\kappa t)^{n}}{n}\left(\log ^{m} t-\frac{m}{n} \log ^{m-1} t+\frac{m(m-1)}{n^{2}} \log ^{m-2} t-\cdots+\frac{(-1)^{m} m !}{n^{m}}\right) .
$$

Taking this fact into account, we set

$$
\begin{aligned}
& \mathcal{I}\left[C(\kappa t)^{n} \log ^{m} t\right] \\
& \quad:=C \frac{(\kappa t)^{n}}{n}\left(\log ^{m} t-\frac{m}{n} \log ^{m-1} t+\frac{m(m-1)}{n^{2}} \log ^{m-2} t-\cdots+\frac{(-1)^{m} m !}{n^{m}}\right)
\end{aligned}
$$

for $(m, n) \in(\mathbb{N} \cup\{0\}) \times \mathbb{N}$ and $C \in M_{2}\left(\mathbb{C}_{\theta}\right)$, which induces a linear operator $\mathcal{I}: \widehat{\mathfrak{L}} \rightarrow \widehat{\mathfrak{L}}$. For $m \leq 2 n$,

$$
\begin{aligned}
& \left\|\mathcal{I}\left[C(\kappa t)^{n} \log ^{m} t\right]\right\| \\
& =\|C\| \frac{|\kappa t|^{n}}{n}\left(|t|^{-m / 4}+\frac{m}{n}|t|^{-(m-1) / 4}+\frac{m(m-1)}{n^{2}}|t|^{-(m-2) / 4}+\cdots+\frac{m !}{n^{m}}\right) \\
& \leq\|C\||\kappa t|^{n}|t|^{-m / 4}\left(1+2|t|^{1 / 4}+\cdots+2^{m}|t|^{m / 4}\right) \\
& \leq\|C\||\kappa t|^{n}|t|^{-m / 4}\left(1-2|t|^{1 / 4}\right)^{-1} \leq 2\|C\||\kappa t|^{n}|t|^{-m / 4}=2\left\|C(\kappa t)^{n} \log ^{m} t\right\|
\end{aligned}
$$

Proposition 4.3. Suppose that $\Phi \in \mathfrak{L}(D)$. Then
(i) $\mathcal{I}[\Phi] \in \mathfrak{L}(D)$,
(ii) $\|\mathcal{I}[\Phi]\| \leq 2\|\Phi\|$,
(iii) $t(d / d t) \mathcal{I}[\Phi]=\Phi$

provided that $|t|<2^{-8}$ for every $(\kappa, t) \in D$.

In what follows we suppose that $|t|<2^{-8}$ for every $(\kappa, t) \in D$.

Lemma 4.4. Let $\Lambda \in M_{2}\left(\mathbb{C}_{\theta}\right)$ and $T \in G L_{2}\left(\mathbb{C}_{\theta}\right)$ satisfy $\left(\mathrm{P} .2^{\prime}\right)$, that is, $T^{-1} \Lambda T=\Delta$. Then, for every $\Phi \in \mathfrak{L}(D) \cup M_{2}\left(\mathbb{C}_{\theta}\right)$, we have $\kappa t \cdot t^{\mp \Lambda} \Phi t^{ \pm \Lambda} \in \mathfrak{L}(D)$ and

$$
\left\|\kappa t \cdot t^{\mp \Lambda} \Phi t^{ \pm \Lambda}\right\| \leq 2\|T\|^{2}\left\|T^{-1}\right\|^{2}|\kappa t||t|^{-1 / 2}\|\Phi\| .
$$


Proof. If $C \in M_{2}\left(\mathbb{C}_{\theta}\right)$, then

$$
\begin{aligned}
\kappa t \cdot t^{\mp \Lambda} C t^{ \pm \Lambda} & =\kappa t T t^{\mp \Delta} T^{-1} C T t^{ \pm \Delta} T^{-1} \\
& =\kappa t T(I \mp \Delta \log t) T^{-1} C T(I \pm \Delta \log t) T^{-1} \\
& =\kappa t\left(C \pm\left[C, T \Delta T^{-1}\right] \log t-T \Delta T^{-1} C T \Delta T^{-1} \log ^{2} t\right) \in \mathfrak{L}(D) .
\end{aligned}
$$

Furthermore, for $(\kappa, t) \in D$,

$$
\begin{aligned}
\left\|\kappa t \cdot t^{\mp \Lambda} C t^{ \pm \Lambda}\right\| & \leq\|C\||\kappa t|\left(1+2\|T\|\left\|T^{-1}\right\||t|^{-1 / 4}+\|T\|^{2}\left\|T^{-1}\right\|^{2}|t|^{-1 / 2}\right) \\
& \leq\|C\||\kappa t||t|^{-1 / 2}\|T\|^{2}\left\|T^{-1}\right\|^{2}\left(1+|t|^{1 / 4}\right)^{2} \\
& \leq 2\|T\|^{2}\left\|T^{-1}\right\|^{2}|\kappa t||t|^{-1 / 2}\|C\| .
\end{aligned}
$$

For the series $\Phi=\sum_{n=1}^{\infty} \sum_{m=0}^{2 n} C_{m}^{n}(\kappa t)^{n} \log ^{m} t \in \mathfrak{L}(D)$, writing

$$
\kappa t \cdot t^{\mp \Lambda} \Phi t^{ \pm \Lambda}=\sum_{n=1}^{\infty} \sum_{m=0}^{2 n} \Xi_{m}^{ \pm, n}(\kappa t)^{n} \log ^{m} t
$$

with

$$
\begin{aligned}
\Xi_{m}^{ \pm, n} & =\kappa t \cdot t^{\mp \Lambda} C_{m}^{n} t^{ \pm \Lambda} \\
& =\kappa t\left(C_{m}^{n} \pm\left[C_{m}^{n}, T \Delta T^{-1}\right] \log t-T \Delta T^{-1} C_{m}^{n} T \Delta T^{-1} \log ^{2} t\right) \in \mathfrak{L}(D),
\end{aligned}
$$

and using the inequality in the matrix case, we find

$$
\begin{aligned}
\left\|\kappa t \cdot t^{\mp \Lambda} \Phi t^{ \pm \Lambda}\right\| & \leq \sum_{n=1}^{\infty} \sum_{m=0}^{2 n}\left\|\Xi_{m}^{ \pm, n}\right\|\left\|I(\kappa t)^{n} \log ^{m} t\right\| \\
& \leq \sum_{n=1}^{\infty} \sum_{m=0}^{2 n} 2\|T\|^{2}\left\|T^{-1}\right\|^{2}|\kappa t||t|^{-1 / 2}\left\|C_{m}^{n}\right\||\kappa t|^{n}|t|^{-m / 4} \\
& =2\|T\|^{2}\left\|T^{-1}\right\|^{2}|\kappa t||t|^{-1 / 2}\|\Phi\|
\end{aligned}
$$

which implies the lemma.

Lemma 4.5. Suppose that $\Lambda \in M_{2}\left(\mathbb{C}_{\theta}\right)$ and $T \in G L_{2}\left(\mathbb{C}_{\theta}\right)$ satisfy $\left(\mathrm{P} .2^{\prime}\right)$, and that $\Phi, \Psi \in \mathfrak{L}(D) \cup M_{2}\left(\mathbb{C}_{\theta}\right)$. Then

$$
\begin{aligned}
& t^{-\Lambda} \mathcal{I}\left[\kappa t \cdot t^{\Lambda} \Phi t^{-\Lambda} \Psi\right] t^{\Lambda} \in \mathfrak{L}(D), \\
& \left\|t^{-\Lambda} \mathcal{I}\left[\kappa t \cdot t^{\Lambda} \Phi t^{-\Lambda} \Psi\right] t^{\Lambda}\right\| \leq 100\|T\|^{3}\left\|T^{-1}\right\|^{3}|\kappa t||t|^{-1 / 2}\|\Phi\|\|\Psi\| .
\end{aligned}
$$

The same relation and estimate are valid for $t^{-\Lambda} \mathcal{I}\left[\kappa t \Phi t^{\Lambda} \Psi t^{-\Lambda}\right] t^{\Lambda}$.

Proof. Write the primitive function of $t^{-1}(\kappa t)^{n} \log ^{m} t$ in the form $(\kappa t)^{n} l_{n}(m, t) / n$ with

$$
l_{n}(m, t):=\log ^{m} t-\frac{m}{n} \log ^{m-1} t+\frac{m(m-1)}{n^{2}} \log ^{m-2} t-\cdots+\frac{(-1)^{m} m !}{n^{m}} .
$$


Suppose that $\Phi, \Psi \in \mathfrak{L}(D) \cup M_{2}\left(\mathbb{C}_{\theta}\right)$ are the monomials

$$
\Phi=C(\kappa t)^{n} \log ^{m} t, \quad \Psi=\tilde{C}(\kappa t)^{n^{\prime}} \log ^{m^{\prime}} t, \quad C, \tilde{C} \in M_{2}\left(\mathbb{C}_{\theta}\right),
$$

where $n \geq 0, m \leq 2 n, n^{\prime} \geq 0, m^{\prime} \leq 2 n^{\prime}$. Since

$$
\kappa t \cdot t^{\Delta} \Phi t^{-\Delta} \Psi=\left(C+[\Delta, C] \log t-\Delta C \Delta \log ^{2} t\right) \tilde{C}(\kappa t)^{\bar{n}+1} \log ^{\bar{m}} t,
$$

we have

$$
\begin{aligned}
& \mathcal{I}\left[\kappa t \cdot t^{\Delta} \Phi t^{-\Delta} \Psi\right] \\
& =\frac{(\kappa t)^{\bar{n}+1}}{\bar{n}+1}\left(C l_{\bar{n}+1}(\bar{m}, t)+[\Delta, C] l_{\bar{n}+1}(\bar{m}+1, t)-\Delta C \Delta l_{\bar{n}+1}(\bar{m}+2, t)\right) \tilde{C},
\end{aligned}
$$

where $\bar{n}=n+n^{\prime}, \bar{m}=m+m^{\prime}$. Hence

$$
t^{-{ }^{\Delta}} \mathcal{I}\left[\kappa t \cdot t^{\Delta} \Phi t^{-\Delta} \Psi\right] t^{\Delta}=\frac{(\kappa t)^{\bar{n}}}{\bar{n}+1} C_{\bar{n}, \bar{m}}^{*}(t) \kappa t \cdot t^{-\Delta} \tilde{C} t^{\Delta}
$$

with

$$
\begin{aligned}
C_{\bar{n}, \bar{m}}^{*}(t) & =t^{-\Delta}\left(C l_{\bar{n}+1}(\bar{m}, t)+[\Delta, C] l_{\bar{n}+1}(\bar{m}+1, t)-\Delta C \Delta l_{\bar{n}+1}(\bar{m}+2, t)\right) t^{\Delta} \\
& =C l_{\bar{n}+1}(\bar{m}, t)+[\Delta, C] \chi_{1}(t)-\Delta C \Delta \chi_{2}(t),
\end{aligned}
$$

where

$$
\begin{aligned}
\chi_{1}(t) & =l_{\bar{n}+1}(\bar{m}+1, t)-l_{\bar{n}+1}(\bar{m}, t) \log t \\
& =\left(-\frac{\bar{m}+1}{\bar{n}+1}+\frac{\bar{m}}{\bar{n}+1}\right) \log ^{\bar{m}} t+\left(\frac{(\bar{m}+1) \bar{m}}{(\bar{n}+1)^{2}}-\frac{\bar{m}(\bar{m}-1)}{(\bar{n}+1)^{2}}\right) \log ^{\bar{m}-1} t+\cdots, \\
\chi_{2}(t) & =l_{\bar{n}+1}(\bar{m}+2, t)-2 l_{\bar{n}+1}(\bar{m}+1, t) \log t+l_{\bar{n}+1}(\bar{m}, t) \log ^{2} t \\
& =\left(\frac{(\bar{m}+2)(\bar{m}+1)}{(\bar{n}+1)^{2}}-2 \frac{(\bar{m}+1) \bar{m}}{(\bar{n}+1)^{2}}+\frac{\bar{m}(\bar{m}-1)}{(\bar{n}+1)^{2}}\right) \log ^{\bar{m}} t+\cdots,
\end{aligned}
$$

and $\bar{m} \leq 2 \bar{n}$. This together with Lemma 4.4 implies

$$
t^{-{ }^{\Delta}} \mathcal{I}\left[\kappa t \cdot t^{\Delta} \Phi t^{-\Delta} \Psi\right] t^{\Delta} \in \mathfrak{L}(D) .
$$

Using $\bar{m}+2 \leq 2 \bar{n}+2=2(\bar{n}+1)$, we have

$$
\begin{aligned}
& \left|l_{\bar{n}+1}(\bar{m}, t)\right| \leq|t|^{-\bar{m} / 4}\left(1+2|t|^{1 / 4}+2^{2}|t|^{1 / 2}+\cdots\right) \leq 2|t|^{-\bar{m} / 4} \\
& \left|\chi_{1}(t)\right| \leq 2|t|^{-\bar{m} / 4}\left(2+2^{2}|t|^{1 / 4}+2^{3}|t|^{1 / 2}+\cdots\right) \leq 8|t|^{-\bar{m} / 4} \\
& \left|\chi_{2}(t)\right| \leq 4|t|^{-\bar{m} / 4}\left(2^{2}+2^{3}|t|^{1 / 4}+2^{4}|t|^{1 / 2}+\cdots\right) \leq 32|t|^{-\bar{m} / 4}
\end{aligned}
$$


for $|t|<2^{-8}$, so that $\left\|(\kappa t)^{\bar{n}} C_{\bar{n}, \bar{m}}^{*}(t)\right\| \leq 50\|C\||\kappa t|^{\bar{n}}|t|^{-\bar{m} / 4}$. This combined with $\left\|\kappa t \cdot t^{-\Delta} \tilde{C} t^{\Delta}\right\| \leq 2\|\tilde{C}\||\kappa t||t|^{-1 / 2}$, which follows from Lemma 4.4 with $T=I$, gives

$$
\begin{aligned}
\left\|t^{-\Delta} \mathcal{I}\left[\kappa t \cdot t^{\Delta} \Phi t^{-\Delta} \Psi\right] t^{\Delta}\right\| & \leq 100\|C\|\|\tilde{C}\||\kappa t|^{\bar{n}+1}|t|^{-\bar{m} / 4-1 / 2} \\
& =100|\kappa t||t|^{-1 / 2}\|\Phi\|\|\Psi\| .
\end{aligned}
$$

By $\left(\right.$ P. $\left.2^{\prime}\right)$,

$$
t^{-\Lambda} \mathcal{I}\left[\kappa t \cdot t^{\Lambda} \Phi t^{-\Lambda} \Psi\right] t^{\Lambda}=T t^{-\Delta} \mathcal{I}\left[\kappa t \cdot t^{\Delta} T^{-1} \Phi T t^{-{ }^{-}} T^{-1} \Psi T\right] t^{\Delta} T^{-1},
$$

which yields the conclusion of the lemma for the monomial case. For the series $\Phi=$ $\sum_{n, m} C_{m}^{n}(\kappa t)^{n} \log ^{m} t, \Psi=\sum_{n^{\prime}, m^{\prime}} \tilde{C}_{m^{\prime}}^{n^{\prime}}(\kappa t)^{n^{\prime}} \log ^{m^{\prime}} t \in \mathfrak{L}(D) \cup M_{2}\left(\mathbb{C}_{\theta}\right)$, observing that

$$
\begin{aligned}
\Upsilon & :=t^{-\Lambda} \mathcal{I}\left[\kappa t \cdot t^{\Lambda}\left(\sum_{n, m} C_{m}^{n}(\kappa t)^{n} \log ^{m} t\right) t^{-\Lambda}\left(\sum_{n^{\prime}, m^{\prime}} \tilde{C}_{m^{\prime}}^{n^{\prime}}(\kappa t)^{n^{\prime}} \log ^{m^{\prime}} t\right)\right] t^{\Lambda} \\
& =\sum_{n, m} \sum_{n^{\prime}, m^{\prime}} t^{-\Lambda} \mathcal{I}\left[\kappa t \cdot t^{\Lambda}\left(C_{m}^{n}(\kappa t)^{n} \log ^{m} t\right) t^{-\Lambda} \tilde{C}_{m^{\prime}}^{n^{\prime}}(\kappa t)^{n^{\prime}} \log ^{m^{\prime}} t\right] t^{\Lambda},
\end{aligned}
$$

we have

$$
\begin{aligned}
\|\Upsilon\| & \leq \sum_{n, m} \sum_{n^{\prime}, m^{\prime}}\left\|t^{-\Lambda} \mathcal{I}\left[\kappa t \cdot t^{\Lambda}\left(C_{m}^{n}(\kappa t)^{n} \log ^{m} t\right) t^{-\Lambda} \tilde{C}_{m^{\prime}}^{n^{\prime}}(\kappa t)^{n^{\prime}} \log ^{m^{\prime}} t\right] t^{\Lambda}\right\| \\
& \leq 100\|T\|^{3}\left\|T^{-1}\right\|^{3}|\kappa t||t|^{-1 / 2} \sum_{n, m}\left\|C_{m}^{n}\right\||\kappa t|^{n}|t|^{-m / 4} \sum_{n^{\prime}, m^{\prime}}\left\|C_{m^{\prime}}^{n^{\prime}}\right\||\kappa t|^{n^{\prime}}|t|^{-m^{\prime} / 4} \\
& \leq 100\|T\|^{3}\left\|T^{-1}\right\|^{3}|\kappa t||t|^{-1 / 2}\|\Phi\|\|\Psi\| .
\end{aligned}
$$

Thus we obtain the lemma.

Remark 4.1. In the case where $\Phi, \Psi \in \widehat{\mathfrak{L}} \cup M_{2}\left(\mathbb{C}_{\theta}\right)$ as well we may show the relations $\kappa t \cdot t^{\mp \Lambda} \Phi t^{ \pm \Lambda}, t^{-\Lambda} \mathcal{I}\left[\kappa t \cdot t^{\Lambda} \Phi t^{-\Lambda} \Psi\right] t^{\Lambda}, t^{-\Lambda} \mathcal{I}\left[\kappa t \Phi t^{\Lambda} \Psi t^{-\Lambda}\right] t^{\Lambda} \in \widehat{\mathfrak{L}}$ by the same arguments as in the proofs of Lemmas 4.4 and 4.5.

Let $\widehat{\mathfrak{L}}^{*}$ be the subring of $\widehat{\mathfrak{L}}$ consisting of formal series of the form

$$
\Phi=\sum_{n=1}^{\infty} \sum_{m=0}^{n} C_{m}^{n}(\kappa t)^{n} \log ^{m} t, \quad C_{m}^{n} \in M_{2}\left(\mathbb{C}_{\theta}\right) .
$$

Lemma 4.6. If $\Phi, \kappa t \Psi$ (respectively, $\kappa t \Phi, \Psi) \in \widehat{\mathfrak{L}}^{*}$, then $\kappa t \cdot t^{\Lambda} \mathcal{I}\left[t^{-\Lambda} \Phi \Psi t^{\Lambda}\right] t^{-\Lambda}$, $\mathcal{I}[\kappa t \Phi \Psi] \in \widehat{\mathfrak{L}}^{*}$, where $\Lambda$ is a matrix as in Lemma 4.5 .

Proof. Let $\Phi=C(\kappa t)^{n} \log ^{m} t$ and $\Psi=\tilde{C}(\kappa t)^{n^{\prime}-1} \log ^{m^{\prime}} t$ be given monomials such that $\Phi, \kappa t \Psi \in \widehat{\mathfrak{L}}^{*}$, where $n \geq 1, m \leq n, n^{\prime} \geq 1, m^{\prime} \leq n^{\prime}$. By the same argument as 
in the proof of Lemma 4.5 we have

$$
\begin{aligned}
\kappa t \cdot t^{\Delta} \mathcal{I}\left[t^{-\Delta}\right. & \left.\Phi \Psi t^{\Delta}\right] t^{-\Delta} \\
& =\frac{(\kappa t)^{\bar{n}}}{\bar{n}-1}\left(C \tilde{C} l_{\bar{n}-1}(\bar{m}, t)-[\Delta, C \tilde{C}] \tilde{\chi}_{1}(t)-\Delta C \tilde{C} \Delta \tilde{\chi}_{2}(t)\right) \in \widehat{\mathfrak{L}}^{*}
\end{aligned}
$$

where

$$
\begin{aligned}
& \tilde{\chi}_{1}(t)=l_{\bar{n}-1}(\bar{m}+1, t)-l_{\bar{n}-1}(\bar{m}, t) \log t=-\frac{\log ^{\bar{m}} t}{\bar{n}-1}+\cdots \\
& \tilde{\chi}_{2}(t)=l_{\bar{n}-1}(\bar{m}+2, t)-2 l_{\bar{n}-1}(\bar{m}+1, t) \log t+l_{\bar{n}-1}(\bar{m}, t) \log ^{2} t=\frac{2 \log ^{\bar{m}} t}{(\bar{n}-1)^{2}}+\cdots \\
& \bar{n}=n+n^{\prime} \geq 2, \bar{m}=m+m^{\prime} \leq \bar{n} . \text { From this the lemma follows. }
\end{aligned}
$$

\section{§4.2. Complex power type}

Let $\widehat{\mathfrak{S}}$ be the ring of formal series of the form

$$
\Phi=\Phi(\sigma, \kappa, t)=\sum_{n=1}^{\infty} \sum_{m=-n}^{n} C_{m}^{n}(\kappa t)^{n} t^{\sigma m}, \quad C_{m}^{n}=C_{m}^{n}(\sigma) \in M_{2}\left(\mathbb{C}_{\theta}(\sigma)\right),
$$

with the additional parameter $\sigma \neq 0$, and set, for $\Phi \in \widehat{\mathfrak{S}}$ as above,

$$
\|\Phi\|=\|\Phi\|(\sigma, \kappa, t):=\sum_{n=1}^{\infty} \sum_{m=-n}^{n}\left\|C_{m}^{n}\right\||\kappa t|^{n}\left|t^{\sigma}\right|^{m} .
$$

Let $\Sigma$ be a domain such that $\Sigma \subset \mathbb{C} \backslash\{0\}$. For a domain $D(\Sigma) \subset \Sigma \times(\mathbb{C} \backslash\{0\}) \times$ $\mathcal{R}(\mathbb{C} \backslash\{0\})$, let

$$
\mathfrak{S}(D(\Sigma)):=\left\{\Phi \in \widehat{\mathfrak{S}} ;\|\Phi\|<\infty \text { for }(\sigma, \kappa, t) \in D(\Sigma) \text { and for }\left(\theta_{\iota}\right) \text { satisfying }(2.4)\right\} .
$$

Proposition 4.7. (1) If $\Phi \in \mathfrak{S}(D(\Sigma))$, then, for each $\left(\theta_{\iota}\right), \Phi=\Phi(\sigma, \kappa, t)$ is holomorphic in $(\sigma, \kappa, t) \in D(\Sigma)$, and satisfies $\|\Phi(\sigma, \kappa, t)\|_{\text {mat }} \leq\|\Phi\|(\sigma, \kappa, t)$.

(2) $\|\Phi\| \equiv 0$ if and only if $\Phi \equiv 0$.

(3) Let $\Phi, \Psi \in \mathfrak{S}(D(\Sigma))$. Then $\Phi+\Psi, \Phi \Psi \in \mathfrak{S}(D(\Sigma))$, and

$$
\|\Phi+\Psi\| \leq\|\Phi\|+\|\Psi\|, \quad\|\Phi \Psi\| \leq\|\Phi\|\|\Psi\| .
$$

If $C \in M_{2}\left(\mathbb{C}_{\theta}(\sigma)\right)$ (respectively, $\left.c \in \mathbb{C}_{\theta}(\sigma)\right)$ satisfies $\|C\|<\infty$ (respectively, $|c|<\infty)$ for $\sigma \in \Sigma$, then $C \Phi, \Phi C \in \mathfrak{S}(D(\Sigma)$ ) (respectively, $c \Phi \in \mathfrak{S}(D(\Sigma))$ ), and

$$
\|C \Phi\|,\|\Phi C\| \leq\|C\|\|\Phi\| \quad \text { (respectively, }\|c \Phi\|=|c|\|\Phi\|) \text {. }
$$


(4) Let $\Phi \in \mathfrak{S}(D(\Sigma))$, and let $\varphi(\tau)=\sum_{n=0}^{\infty} \varphi_{n} \tau^{n}$ with $\varphi_{n} \in \mathbb{C}$. If $|\varphi|(|\kappa t|)=$ $\sum_{n=0}^{\infty}\left|\varphi_{n}\right||\kappa t|^{n}<\infty$ in $D(\Sigma)$, then $\varphi(\kappa t) \Phi \in \mathfrak{S}(D(\Sigma))$ and

$$
\|\varphi(\kappa t) \Phi\| \leq|\varphi|(|\kappa t|)\|\Phi\| .
$$

Proof. Suppose that $\Phi(\sigma, \kappa, t)=\sum_{n=1}^{\infty} \sum_{m=-n}^{n} C_{m}^{n}(\kappa t)^{n} t^{\sigma m} \in \mathfrak{S}(D(\Sigma))$. For any $(\tilde{\sigma}, \tilde{\kappa}, \tilde{t}) \in D(\Sigma)$, we may choose a neighbourhood $D_{0} \subset D(\Sigma)$ of $(\tilde{\sigma}, \tilde{\kappa}, \tilde{t})$, a point $\left(\sigma_{0}, \kappa_{0}, t_{0}\right) \in D(\Sigma) \backslash D_{0}$, and a positive number $\varepsilon_{*}$ in such a way that $\left|\kappa t^{1-\sigma}\right| \leq$ $\left(1-\varepsilon_{*}\right)\left|\kappa_{0} t_{0}^{1-\sigma_{0}}\right|,\left|t^{\sigma}\right| \leq\left(1-\varepsilon_{*}\right)\left|t_{0}^{\sigma_{0}}\right|$ for every $(\sigma, \kappa, t) \in D_{0}$, since the mapping $(\kappa, \sigma, t) \mapsto\left(\kappa, \kappa t^{1-\sigma}, t^{\sigma}\right)$ is biholomorphic. Observing that

$$
\|\Phi\|(\sigma, \kappa, t)=\sum_{n=1}^{\infty} \sum_{m=-n}^{n}\left\|C_{m}^{n}\right\||\kappa t|^{n}\left|t^{\sigma}\right|^{m}=\sum_{n=1}^{\infty} \sum_{m=-n}^{n}\left\|C_{m}^{n}\right\|\left|\kappa t^{1-\sigma}\right|^{n}\left|t^{\sigma}\right|^{n+m},
$$

from $\|\Phi\|\left(\sigma_{0}, \kappa_{0}, t_{0}\right)<\infty$, we obtain $\left\|C_{m}^{n}\right\|=O\left(\left|\kappa_{0} t_{0}^{1-\sigma_{0}}\right|^{-n}\left|t_{0}^{\sigma_{0}}\right|^{-n-m}\right)$. Hence

$$
\begin{aligned}
\left\|C_{m}^{n}\right\||\kappa t|^{n}\left|t^{\sigma}\right|^{m} & =O\left(\left(\left|\kappa t^{1-\sigma}\right| /\left|\kappa_{0} t_{0}^{1-\sigma_{0}}\right|\right)^{n}\left(\left|t^{\sigma}\right| /\left|t_{0}^{\sigma_{0}}\right|\right)^{n+m}\right) \\
& =O\left(\left(1-\varepsilon_{*}\right)^{2 n+m}\right)=O\left(\left(1-\varepsilon_{*}\right)^{n}\right)
\end{aligned}
$$

in $D_{0}$, which implies the first assertion.

Proposition 4.8. Suppose that each $\Phi_{\nu} \in \mathfrak{S}(D(\Sigma))$ consists of summands with $n \geq \nu$, and that, for each $\left(\theta_{\iota}\right), \sum_{\nu=1}^{\infty}\left\|\Phi_{\nu}\right\|<\infty$ in $D(\Sigma)$. Then $\Phi^{\infty}=\sum_{\nu=1}^{\infty} \Phi_{\nu} \in$ $\mathfrak{S}(D(\Sigma))$, and $\left\|\Phi^{\infty}\right\| \leq \sum_{\nu=1}^{\infty}\left\|\Phi_{\nu}\right\|$.

For $(m, n) \in \mathbb{Z} \times \mathbb{N}$ and $C \in M_{2}\left(\mathbb{C}_{\theta}(\sigma)\right)$, set

$$
\mathcal{I}\left[C(\kappa t)^{n} t^{\sigma m}\right]:=\frac{1}{n+\sigma m} C(\kappa t)^{n} t^{\sigma m},
$$

which is the primitive function of $t^{-1} C(\kappa t)^{n} t^{\sigma m}$. This induces a linear operator $\mathcal{I}: \widehat{\mathfrak{S}} \rightarrow \widehat{\mathfrak{S}}$. Let $\Sigma_{0}$ be as in Theorem 2.1.

Proposition 4.9. If $\Phi \in \mathfrak{S}\left(D\left(\Sigma_{0}\right)\right)$, then
(i) $\mathcal{I}[\Phi] \in \mathfrak{S}\left(D\left(\Sigma_{0}\right)\right)$,
(ii) $\|\mathcal{I}[\Phi]\| \leq L_{0}\|\Phi\|$,
(iii) $t(d / d t) \mathcal{I}[\Phi]=\Phi$,

where $L_{0}$ is a positive constant depending only on $\Sigma_{0}$.

Proof. Set $d_{0}:=\operatorname{dist}\left(\Sigma_{0}, S_{0}\right)>0$. Suppose that $\sigma \in \Sigma_{0}$. If $1 \leq|m| \leq n$, then $|n+\sigma m|=|m||\sigma+n / m| \geq d_{0}$. Hence, for $\Phi=\sum_{n=1}^{\infty} \sum_{m=-n}^{n} C_{m}^{n}(\kappa t)^{n} t^{\sigma m} \in$ $\mathfrak{S}\left(D\left(\Sigma_{0}\right)\right)$

$$
\|\mathcal{I}[\Phi]\|=\sum_{n=1}^{\infty} \sum_{m=-n}^{n} \frac{\left\|C_{m}^{n}\right\|}{|n+\sigma m|}|\kappa t|^{n}\left|t^{\sigma}\right|^{m} \leq \max \left\{1,1 / d_{0}\right\}\|\Phi\|,
$$

which implies the proposition. 
Lemma 4.10. Let $\Lambda \in M_{2}\left(\mathbb{C}_{\theta}\left[\sigma, \sigma^{-1}\right]\right)$ and $T \in G L_{2}\left(\mathbb{C}_{\theta}\left[\sigma, \sigma^{-1}\right]\right)$ satisfy (P.2), that is, $T^{-1} \Lambda T=(\sigma / 2) J$. Suppose that $\Phi \in \mathfrak{S}\left(D\left(\Sigma_{0}\right)\right) \cup M_{2}\left(\mathbb{C}_{\theta}(\sigma)\right)$, and that $\|\Phi\|<\infty$ for each $\left(\theta_{\iota}\right)$ and for $\sigma \in \Sigma_{0}$ if $\Phi \in M_{2}\left(\mathbb{C}_{\theta}(\sigma)\right)$. Then $\kappa t \cdot t^{\mp \Lambda} \Phi t^{ \pm \Lambda} \in$ $\mathfrak{S}\left(D\left(\Sigma_{0}\right)\right)$, and

$$
\left\|\kappa t \cdot t^{\mp \Lambda} \Phi t^{ \pm \Lambda}\right\| \leq 2\|T\|^{2}\left\|T^{-1}\right\|^{2}|\kappa t|\left(\left|t^{\sigma}\right|+\left|t^{-\sigma}\right|\right)\|\Phi\| .
$$

Proof. If $\Phi=C \in M_{2}\left(\mathbb{C}_{\theta}(\sigma)\right)$, then

$$
\begin{aligned}
\kappa t \cdot t^{\mp \Lambda} C t^{ \pm \Lambda} & =\kappa t T t^{\mp(\sigma / 2) J} T^{-1} C T t^{ \pm(\sigma / 2) J} T^{-1} \\
& =\kappa t T\left(\begin{array}{cc}
\left(T^{-1} C T\right)_{11} & \left(T^{-1} C T\right)_{12} t^{\mp \sigma} \\
\left(T^{-1} C T\right)_{21} t^{ \pm \sigma} & \left(T^{-1} C T\right)_{22}
\end{array}\right) T^{-1} \in \mathfrak{S}\left(D\left(\Sigma_{0}\right)\right) .
\end{aligned}
$$

Hence, for $(\sigma, \kappa, t) \in D\left(\Sigma_{0}\right)$,

$$
\begin{aligned}
\left\|\kappa t \cdot t^{\mp \Lambda} C t^{ \pm \Lambda}\right\| & \leq\|T\|\left\|T^{-1}\right\|\left\|T^{-1} C T\right\||\kappa t|\left(1+\left|t^{\sigma}\right|+\left|t^{-\sigma}\right|\right) \\
& \leq 2\|T\|^{2}\left\|T^{-1}\right\|^{2}|\kappa t|\left(\left|t^{\sigma}\right|+\left|t^{-\sigma}\right|\right)\|C\| .
\end{aligned}
$$

For the series $\Phi=\sum_{n=1}^{\infty} \sum_{m=-n}^{n} C_{m}^{n}(\kappa t)^{n} t^{\sigma m} \in \mathfrak{S}\left(D\left(\Sigma_{0}\right)\right)$, writing

$$
\kappa t \cdot t^{\mp \Lambda} \Phi t^{ \pm \Lambda}=\sum_{n=1}^{\infty} \sum_{m=-n}^{n} \Xi_{m}^{ \pm, n}(\kappa t)^{n} t^{\sigma m}
$$

with $\Xi_{m}^{ \pm, n}=\kappa t \cdot t^{\mp \Lambda} C_{m}^{n} t^{ \pm \Lambda}$ and using the inequality in the matrix case, we obtain the lemma.

Lemma 4.11. Let $\Lambda \in M_{2}\left(\mathbb{C}_{\theta}\left[\sigma, \sigma^{-1}\right]\right)$ and $T \in G L_{2}\left(\mathbb{C}_{\theta}\left[\sigma, \sigma^{-1}\right]\right)$ satisfy (P.2). Suppose that $\Phi, \Psi \in \mathfrak{S}\left(D\left(\Sigma_{0}\right)\right) \cup M_{2}\left(\mathbb{C}_{\theta}(\sigma)\right)$, and that $\|\Phi\|$ (respectively, $\|\Psi\|$ ) $<\infty$ for each $\left(\theta_{\iota}\right)$ and for $\sigma \in \Sigma_{0}$ if $\Phi$ (respectively, $\left.\Psi\right) \in M_{2}\left(\mathbb{C}_{\theta}(\sigma)\right)$. Then $t^{-\Lambda} \mathcal{I}\left[\kappa t \cdot t^{\Lambda} \Phi t^{-\Lambda} \Psi\right] t^{\Lambda} \in \mathfrak{S}\left(D\left(\Sigma_{0}\right)\right)$, and

$$
\left\|t^{-\Lambda} \mathcal{I}\left[\kappa t \cdot t^{\Lambda} \Phi t^{-\Lambda} \Psi\right] t^{\Lambda}\right\| \leq \tilde{L}_{0}\|T\|^{3}\left\|T^{-1}\right\|^{3}|\kappa t|\left(\left|t^{\sigma}\right|+\left|t^{-\sigma}\right|\right)\|\Phi\|\|\Psi\|,
$$

where $\tilde{L}_{0}$ is a positive constant depending only on $\Sigma_{0}$. The same relation and estimate are valid for $t^{-\Lambda} \mathcal{I}\left[\kappa t \Phi t^{\Lambda} \Psi t^{-\Lambda}\right] t^{\Lambda}$.

Proof. Consider the case where $\Phi=C(\kappa t)^{n} t^{\sigma m}, \Psi=\tilde{C}(\kappa t)^{n^{\prime}} t^{\sigma m^{\prime}}$ with $C, \tilde{C} \in$ $M_{2}\left(\mathbb{C}_{\theta}(\sigma)\right), n \geq 0,|m| \leq n, n^{\prime} \geq 0,\left|m^{\prime}\right| \leq n^{\prime}$. Observing that

$$
t^{(\sigma / 2) J} \Phi t^{-(\sigma / 2) J} \Psi=\left(\begin{array}{cc}
C_{11}(\kappa t)^{n+n^{\prime}} t^{\sigma\left(m+m^{\prime}\right)} & C_{12}(\kappa t)^{n+n^{\prime}} t^{\sigma\left(m+m^{\prime}+1\right)} \\
C_{21}(\kappa t)^{n+n^{\prime}} t^{\sigma\left(m+m^{\prime}-1\right)} & C_{22}(\kappa t)^{n+n^{\prime}} t^{\sigma\left(m+m^{\prime}\right)}
\end{array}\right) \tilde{C},
$$

we have

$$
\begin{aligned}
\Upsilon & :=t^{-(\sigma / 2) J} \mathcal{I}\left[\kappa t \cdot t^{(\sigma / 2) J} \Phi t^{-(\sigma / 2) J} \Psi\right] t^{(\sigma / 2) J} \\
& =C_{n, n^{\prime}, m, m^{\prime}}^{*} \kappa t \cdot t^{-(\sigma / 2) J}\left(\tilde{C}(\kappa t)^{n+n^{\prime}} t^{\sigma\left(m+m^{\prime}\right)}\right) t^{(\sigma / 2) J}
\end{aligned}
$$


with

$$
\begin{aligned}
& C_{n, n^{\prime}, m, m^{\prime}}^{*} \\
& \quad=\left(\begin{array}{cc}
C_{11}\left(n+n^{\prime}+1+\sigma\left(m+m^{\prime}\right)\right)^{-1} & C_{12}\left(n+n^{\prime}+1+\sigma\left(m+m^{\prime}+1\right)\right)^{-1} \\
C_{21}\left(n+n^{\prime}+1+\sigma\left(m+m^{\prime}-1\right)\right)^{-1} & C_{22}\left(n+n^{\prime}+1+\sigma\left(m+m^{\prime}\right)\right)^{-1}
\end{array}\right)
\end{aligned}
$$

satisfying $\left\|C_{n, n^{\prime}, m, m^{\prime}}^{*}\right\| \leq \max \left\{1,1 / d_{0}\right\}\|C\|$, where $d_{0}=\operatorname{dist}\left(\Sigma_{0}, S_{0}\right)$. By Lemma 4.10 this gives $\Upsilon \in \mathfrak{S}\left(D\left(\Sigma_{0}\right)\right)$. Furthermore, by Lemma 4.10,

$$
\begin{aligned}
\|\Upsilon\| & \leq 2\left\|C_{n, n^{\prime}, m, m^{\prime}}^{*}\right\||\kappa t|\left(\left|t^{\sigma}\right|+\left|t^{-\sigma}\right|\right)\left\|\tilde{C}(\kappa t)^{n+n^{\prime}} t^{\sigma\left(m+m^{\prime}\right)}\right\| \\
& \leq 2 \max \left\{1,1 / d_{0}\right\}\|C\|\|\tilde{C}\||\kappa t|^{n+n^{\prime}+1}\left|t^{\sigma}\right|^{m+m^{\prime}}\left(\left|t^{\sigma}\right|+\left|t^{-\sigma}\right|\right) \\
& =2 \max \left\{1,1 / d_{0}\right\}|\kappa t|\left(\left|t^{\sigma}\right|+\left|t^{-\sigma}\right|\right)\|\Phi\|\|\Psi\|,
\end{aligned}
$$

which leads to the desired estimate in the monomial case. For given series $\Phi=$ $\sum_{n, m} C_{m}^{n}(\kappa t)^{n} t^{\sigma m}, \Psi=\sum_{n^{\prime}, m^{\prime}} \tilde{C}_{m^{\prime}}^{n^{\prime}}(\kappa t)^{n^{\prime}} t^{\sigma m^{\prime}} \in \mathfrak{S}\left(D\left(\Sigma_{0}\right)\right) \cup M_{2}\left(\mathbb{C}_{\theta}(\sigma)\right)$, we can derive the conclusion by the same argument as in the proof of Lemma 4.5.

Suppose that $\sigma_{0} \in \Sigma_{+}$, where $\Sigma_{+}$is as in Theorem 2.1. Let $\widehat{\mathfrak{S}}^{+}\left(\sigma_{0}\right)$ be the ring of formal series of the form

$$
\Phi=\Phi\left(\sigma_{0}, \kappa, t\right)=\sum_{n=1}^{\infty} \sum_{m=0}^{n} C_{m}^{n}(\kappa t)^{n} t^{\sigma_{0} m}, \quad C_{m}^{n} \in M_{2}\left(\mathbb{C}_{\theta}\left(\sigma_{0}\right)\right) .
$$

For a domain $D\left(\sigma_{0}\right) \subset(\mathbb{C} \backslash\{0\}) \times \mathcal{R}(\mathbb{C} \backslash\{0\})$, let $\mathfrak{S}^{+}\left(D\left(\sigma_{0}\right)\right)$ be the subring consisting of $\Phi \in \widehat{\mathfrak{S}}^{+}\left(\sigma_{0}\right)$ such that

$$
\|\Phi\|=\|\Phi\|\left(\sigma_{0}, \kappa, t\right):=\sum_{n=1}^{\infty} \sum_{m=0}^{n}\left\|C_{m}^{n}\right\||\kappa t|^{n}\left|t^{\sigma_{0}}\right|^{m}<\infty
$$

for $(\kappa, t) \in D\left(\sigma_{0}\right)$ and for $\left(\theta_{\iota}\right)$ satisfying $(2.4)$. Then $\mathfrak{S}^{+}\left(D\left(\sigma_{0}\right)\right)$ with the norm thus defined has properties analogous to those of $\mathfrak{S}(D(\Sigma))$. For example, if $\Phi \in \mathfrak{S}^{+}\left(D\left(\sigma_{0}\right)\right)$, then $\Phi=\Phi\left(\sigma_{0}, \kappa, t\right)$ is holomorphic in $(\kappa, t) \in D\left(\sigma_{0}\right)$, and satisfies

$$
\left\|\Phi\left(\sigma_{0}, \kappa, t\right)\right\|_{\text {mat }} \leq\|\Phi\|\left(\sigma_{0}, \kappa, t\right) .
$$

In this case as well we define $\mathcal{I}[\cdot]$ by $\mathcal{I}\left[C(\kappa t)^{n} t^{\sigma_{0} m}\right]=\left(n+\sigma_{0} m\right)^{-1} C(\kappa t)^{n} t^{\sigma_{0} m}$.

Proposition 4.12. If $\Phi \in \mathfrak{S}^{+}\left(D\left(\sigma_{0}\right)\right)$, then
(i) $\mathcal{I}[\Phi] \in \mathfrak{S}^{+}\left(D\left(\sigma_{0}\right)\right)$,
(ii) $\|\mathcal{I}[\Phi]\| \leq L_{0}^{+}\|\Phi\|$
(iii) $t(d / d t) \mathcal{I}[\Phi]=\Phi$,

where $L_{0}^{+}$is a positive constant depending only on $\sigma_{0}$.

Proof. Set $d_{0}^{+}:=\operatorname{dist}\left(\left\{\sigma_{0}\right\},\{\sigma \leq-1\} \cup \mathbb{Z}\right)>0$. If $1 \leq m \leq n$, then $\left|n+\sigma_{0} m\right|=$ $m\left|\sigma_{0}+n / m\right| \geq d_{0}^{+}$, from which the proposition follows. 
For a formal series of the form

$$
\Phi=\sum_{n=1}^{\infty} \sum_{m=-1}^{n+2} C_{m}^{n}(\kappa t)^{n} t^{\sigma_{0} m}, \quad C_{m}^{n} \in M_{2}\left(\mathbb{C}_{\theta}\left(\sigma_{0}\right)\right),
$$

which does not necessarily belong to $\widehat{\mathfrak{S}}^{+}\left(\sigma_{0}\right)$, we may extend the operator $\mathcal{I}[\cdot]$ in such a way that

$$
\mathcal{I}[\Phi]=\sum_{n=1}^{\infty} \sum_{m=-1}^{n+2} \frac{1}{n+\sigma_{0} m} C_{m}^{n}(\kappa t)^{n} t^{\sigma_{0} m}
$$

as long as $n+\sigma_{0} m \neq 0$ for $(m, n)$ satisfying $n \geq 1,-1 \leq m \leq n+2$. Furthermore, if

$$
\|\mathcal{I}[\Phi]\|=\sum_{n=1}^{\infty} \sum_{m=-1}^{n+2} \frac{1}{\left|n+\sigma_{0} m\right|}\left\|C_{m}^{n}\right\||\kappa t|^{n}\left|t^{\sigma_{0}}\right|^{m}
$$

converges uniformly in a domain, then $t(d / d t) \mathcal{I}[\Phi]=\Phi$. For such an extension of $\mathcal{I}[\cdot]$ we have

Lemma 4.13. Suppose that $\sigma_{0} \in \Sigma_{+} \cap\{\operatorname{Re} \sigma>1\}$, and that $\Lambda \in M_{2}\left(\mathbb{C}_{\theta}\left[\sigma_{0}, \sigma_{0}^{-1}\right]\right)$ and $T \in G L_{2}\left(\mathbb{C}_{\theta}\left[\sigma_{0}, \sigma_{0}^{-1}\right]\right)$ satisfy (P.2) with $\sigma=\sigma_{0}$. If $\Phi, \kappa t \Psi$ (respectively, $\kappa t \Phi, \Psi) \in \mathfrak{S}^{+}\left(D\left(\sigma_{0}\right)\right)$, then

$$
\begin{aligned}
\kappa t \cdot t^{\Lambda} \mathcal{I}\left[t^{-\Lambda} \Phi \Psi t^{\Lambda}\right] t^{-\Lambda} & \in \mathfrak{S}^{+}\left(D\left(\sigma_{0}\right)\right) \\
\left\|\kappa t \cdot t^{\Lambda} \mathcal{I}\left[t^{-\Lambda} \Phi \Psi t^{\Lambda}\right] t^{-\Lambda}\right\| & \leq \tilde{L}_{0}^{+}\|T\|^{3}\left\|T^{-1}\right\|^{3}\|\Phi\|\|\kappa t \Psi\| \\
\text { (respectively, } & \left.\leq \tilde{L}_{0}^{+}\|T\|^{3}\left\|T^{-1}\right\|^{3}\|\kappa t \Phi\|\|\Psi\|\right),
\end{aligned}
$$

where $\tilde{L}_{0}^{+}$is a positive constant depending only on $\sigma_{0}$.

Remark 4.2. In general $t^{-\Lambda} \Phi \Psi t^{\Lambda}$ has the form $\sum_{n=1}^{\infty} \sum_{m=-1}^{n+2} C_{m}^{n}(\kappa t)^{n} t^{\sigma_{0} m}$ with $C_{m}^{n} \in M_{2}\left(\mathbb{C}_{\theta}\left(\sigma_{0}\right)\right)$, which does not necessarily belong to $\widehat{\mathfrak{S}}^{+}\left(\sigma_{0}\right)$.

Proof of Lemma 4.13. For the monomials $\Phi=C(\kappa t)^{n} t^{\sigma_{0} m}$ and $\Psi=\tilde{C}(\kappa t)^{n^{\prime}-1} t^{\sigma_{0} m^{\prime}}$, where $C, \tilde{C} \in M_{2}\left(\mathbb{C}_{\theta}\left(\sigma_{0}\right)\right), n \geq 1,0 \leq m \leq n, n^{\prime} \geq 1,0 \leq m^{\prime} \leq n^{\prime}$, we have

$t^{-\left(\sigma_{0} / 2\right) J} \Phi \Psi t^{\left(\sigma_{0} / 2\right) J}=\left(\begin{array}{cc}C_{11}^{*}(\kappa t)^{n+n^{\prime}-1} t^{\sigma_{0}\left(m+m^{\prime}\right)} & C_{12}^{*}(\kappa t)^{n+n^{\prime}-1} t^{\sigma_{0}\left(m+m^{\prime}-1\right)} \\ C_{21}^{*}(\kappa t)^{n+n^{\prime}-1} t^{\sigma_{0}\left(m+m^{\prime}+1\right)} & C_{22}^{*}(\kappa t)^{n+n^{\prime}-1} t^{\sigma_{0}\left(m+m^{\prime}\right)}\end{array}\right)$

with $C^{*}=\left(C_{i j}^{*}\right)=C \tilde{C}$. Under the condition $\sigma_{0} \in \Sigma_{+} \cap\{\operatorname{Re} \sigma>1\}$, for all $(m, n)$, $\left(m^{\prime}, n^{\prime}\right)$ as above,

$$
\left|\left(n+n^{\prime}-1\right)+\sigma_{0}\left(m+m^{\prime}\right)\right|,\left|\left(n+n^{\prime}-1\right)+\sigma_{0}\left(m+m^{\prime} \pm 1\right)\right| \geq d_{0}^{*}>0,
$$

where $d_{0}^{*}=\min \left\{1, d_{0}^{+}\right\}$with $d_{0}^{+}=\operatorname{dist}\left(\left\{\sigma_{0}\right\},\{\sigma \leq-1\} \cup \mathbb{Z}\right)$. Then

$$
\kappa t \cdot t^{\left(\sigma_{0} / 2\right) J} \mathcal{I}\left[t^{-\left(\sigma_{0} / 2\right) J} \Phi \Psi t^{\left(\sigma_{0} / 2\right) J}\right] t^{-\left(\sigma_{0} / 2\right) J}=C_{n, n^{\prime}, m, m^{\prime}}^{* *}(\kappa t)^{n+n^{\prime}} t^{\sigma_{0}\left(m+m^{\prime}\right)}
$$

with 
$C_{n, n^{\prime}, m, m^{\prime}}^{* *}$

$$
=\left(\begin{array}{cc}
C_{11}^{*}\left(n+n^{\prime}-1+\sigma_{0}\left(m+m^{\prime}\right)\right)^{-1} & C_{12}^{*}\left(n+n^{\prime}-1+\sigma_{0}\left(m+m^{\prime}-1\right)\right)^{-1} \\
C_{21}^{*}\left(n+n^{\prime}-1+\sigma_{0}\left(m+m^{\prime}+1\right)\right)^{-1} & C_{22}^{*}\left(n+n^{\prime}-1+\sigma_{0}\left(m+m^{\prime}\right)\right)^{-1}
\end{array}\right)
$$

satisfying $\left\|C_{n, n^{\prime}, m, m^{\prime}}^{* *}\right\| \leq\left\|C^{*}\right\| / d_{0}^{*} \leq\|C\|\|\tilde{C}\| / d_{0}^{*}$. Using $\kappa t \Psi=\tilde{C}(\kappa t)^{n^{\prime}} t^{\sigma_{0} m^{\prime}}$, we arrive at the conclusion in the monomial case. For series we can verify the lemma by the same argument as in the proof of Lemma 4.5.

Remark 4.3. For formal series in $\widehat{\mathfrak{S}}$ and in $\widehat{\mathfrak{S}}^{+}\left(\sigma_{0}\right)$ the same conclusions as in Lemmas 4.10, 4.11 and 4.13 are valid except for the inequalities. For example, if $\Phi \in \widehat{\mathfrak{S}} \cup M_{2}\left(\mathbb{C}_{\theta}(\sigma)\right)$, then $\kappa t \cdot t^{\mp \Lambda} \Phi t^{ \pm \Lambda} \in \widehat{\mathfrak{S}}$ for $\Lambda$ as in Lemma 4.10.

\section{§5. Proofs of Theorems 2.1 and 2.2}

\section{$\S 5.1$. Schlesinger equation}

Instead of (2.1) itself we consider the system

$$
\begin{aligned}
& t \frac{d A_{0}}{d t}=\left[A_{x}, A_{0}\right], \quad t \frac{d A_{x}}{d t}=\left[A_{0}, A_{x}\right]-\frac{\kappa t}{1-\kappa t}\left[A_{1}, A_{x}\right], \\
& t \frac{d A_{1}}{d t}=-\frac{\kappa t}{1-\kappa t}\left[A_{x}, A_{1}\right],
\end{aligned}
$$

which is obtained by putting $x=\kappa t$ in (2.1). As will be seen, $\kappa$ is essentially one of the integration constants of solutions of (2.1).

Proposition 5.1. Let $\Lambda_{0}, \Lambda_{x}, \Lambda_{1}$ and $\Lambda=\Lambda_{0}+\Lambda_{x}$ be matrices such that $\Lambda_{0}+$ $\Lambda_{x}+\Lambda_{1}=\Lambda+\Lambda_{1}=-\left(\theta_{\infty} / 2\right) J$. Under the supposition

$$
A_{0}+A_{x}+A_{1} \equiv-\left(\theta_{\infty} / 2\right) J,
$$

the change of unknown matrices

$$
t^{-\Lambda} A_{0} t^{\Lambda}=\Lambda_{0}+U_{0}, \quad t^{-\Lambda} A_{x} t^{\Lambda}=\Lambda_{x}+U_{x}, \quad A_{1}=\Lambda_{1}+U_{1}
$$

transforms (5.1) into

$$
\begin{aligned}
& t \frac{d U_{0}}{d t}=-\left[t^{-\Lambda} U_{1} t^{\Lambda}, \Lambda_{0}+U_{0}\right], \\
& t \frac{d U_{x}}{d t}=-\left[t^{-\Lambda} U_{1} t^{\Lambda}, \Lambda_{x}+U_{x}\right]-\frac{\kappa t}{1-\kappa t}\left[t^{-\Lambda}\left(\Lambda_{1}+U_{1}\right) t^{\Lambda}, \Lambda_{x}+U_{x}\right], \\
& t \frac{d U_{1}}{d t}=-\frac{\kappa t}{1-\kappa t}\left[t^{\Lambda}\left(\Lambda_{x}+U_{x}\right) t^{-\Lambda}, \Lambda_{1}+U_{1}\right] .
\end{aligned}
$$

Proof. Set $B_{0}=t^{-\Lambda} A_{0} t^{\Lambda}$ and $B_{x}=t^{-\Lambda} A_{x} t^{\Lambda}$. Then, by (5.1),

$$
t \frac{d B_{0}}{d t}=t^{-\Lambda}\left(t \frac{d A_{0}}{d t}-\Lambda A_{0}+A_{0} \Lambda\right) t^{\Lambda}=t^{-\Lambda}\left(\left[A_{x}, A_{0}\right]-\left[\Lambda, A_{0}\right]\right) t^{\Lambda} .
$$


If $A_{0}+A_{x}+A_{1} \equiv-\left(\sigma_{\infty} / 2\right) J=\Lambda+\Lambda_{1}$, the right-hand member becomes

$$
\left[t^{-\Lambda}\left(A_{x}-\Lambda\right) t^{\Lambda}, t^{-\Lambda} A_{0} t^{\Lambda}\right]=\left[t^{-\Lambda}\left(\Lambda_{1}-A_{0}-A_{1}\right) t^{\Lambda}, B_{0}\right]=\left[t^{-\Lambda}\left(\Lambda_{1}-A_{1}\right) t^{\Lambda}, B_{0}\right] .
$$

Thus we obtain

$$
\begin{aligned}
& t \frac{d B_{0}}{d t}=\left[t^{-\Lambda}\left(\Lambda_{1}-A_{1}\right) t^{\Lambda}, B_{0}\right], \\
& t \frac{d B_{x}}{d t}=\left[t^{-\Lambda}\left(\Lambda_{1}-A_{1}\right) t^{\Lambda}, B_{x}\right]-\frac{\kappa t}{1-\kappa t}\left[t^{-\Lambda} A_{1} t^{\Lambda}, B_{x}\right], \\
& t \frac{d A_{1}}{d t}=-\frac{\kappa t}{1-\kappa t}\left[t^{\Lambda} B_{x} t^{-\Lambda}, A_{1}\right],
\end{aligned}
$$

which is equivalent to (5.1) under the supposition $A_{0}+A_{x}+A_{1} \equiv-\left(\sigma_{\infty} / 2\right) J$. Substitution of $B_{0}=\Lambda_{0}+U_{0}, B_{x}=\Lambda_{x}+U_{x}, A_{1}=\Lambda_{1}+U_{1}$ yields system $(5.2)$.

\section{§5.2. Proof of Theorem 2.2}

The main part of the proof of Theorem 2.2 is solving (5.2) near $t=0$.

5.2.1. Iteration. Let $\Lambda_{0}, \Lambda_{x}, \Lambda_{1} \in M_{2}\left(\mathbb{C}_{\theta}\right), T \in G L_{2}\left(\mathbb{C}_{\theta}\right)$ and $\Lambda=\Lambda_{0}+\Lambda_{x}$ be matrices with the properties (P.1), (P.2'), (P.3). If, for $U_{0}, U_{x}, U_{1} \in \mathfrak{L}(D)$, the right-hand members of system (5.2) also belong to $\mathfrak{L}(D)$ and if the system of relations

$$
\begin{aligned}
& U_{1}=-\mathcal{I}\left[\frac{\kappa t}{1-\kappa t}\left[t^{\Lambda}\left(\Lambda_{x}+U_{x}\right) t^{-\Lambda}, \Lambda_{1}+U_{1}\right]\right] \\
& U_{0}=-\mathcal{I}\left[\left[t^{-\Lambda} U_{1} t^{\Lambda}, \Lambda_{0}+U_{0}\right]\right] \\
& U_{x}=-\mathcal{I}\left[\left[t^{-\Lambda} U_{1} t^{\Lambda}, \Lambda_{x}+U_{x}\right]\right]-\mathcal{I}\left[\frac{\kappa t}{1-\kappa t}\left[t^{-\Lambda}\left(\Lambda_{1}+U_{1}\right) t^{\Lambda}, \Lambda_{x}+U_{x}\right]\right]
\end{aligned}
$$

holds, then $\left(U_{0}, U_{x}, U_{1}\right)$ solves $(5.2)$, where $\mathcal{I}[\cdot]$ is the operator defined in Section 4.1. We would like to construct such series by iteration. Define the sequence $\left\{\left(U_{0}^{(\nu)}, U_{x}^{(\nu)}, U_{1}^{(\nu)}\right) \in(\widehat{\mathfrak{L}})^{3} ; \nu \geq 0\right\}$ by

$$
\begin{aligned}
U_{1}^{(0)}= & U_{0}^{(0)}=U_{x}^{(0)} \equiv 0, \\
U_{1}^{(\nu+1)}= & -\mathcal{I}\left[\frac{\kappa t}{1-\kappa t}\left[t^{\Lambda}\left(\Lambda_{x}+U_{x}^{(\nu)}\right) t^{-\Lambda}, \Lambda_{1}+U_{1}^{(\nu)}\right]\right], \\
U_{0}^{(\nu+1)}= & -\mathcal{I}\left[\left[t^{-\Lambda} U_{1}^{(\nu+1)} t^{\Lambda}, \Lambda_{0}+U_{0}^{(\nu)}\right]\right], \\
U_{x}^{(\nu+1)}= & -\mathcal{I}\left[\left[t^{-\Lambda} U_{1}^{(\nu+1)} t^{\Lambda}, \Lambda_{x}+U_{x}^{(\nu)}\right]\right] \\
& -\mathcal{I}\left[\frac{\kappa t}{1-\kappa t}\left[t^{-\Lambda}\left(\Lambda_{1}+U_{1}^{(\nu+1)}\right) t^{\Lambda}, \Lambda_{x}+U_{x}^{(\nu)}\right]\right] .
\end{aligned}
$$


Note that the right-hand members of the last two relations contain $t^{-\Lambda} U_{1}^{(\nu+1)} t^{\Lambda}$. This procedure is possible, since at each step the series on which $\mathcal{I}[\cdot]$ acts belong to $\widehat{\mathfrak{L}}$. Indeed, we have

Proposition 5.2. $U_{1}^{(\nu)}, t^{-\Lambda} U_{1}^{(\nu)} t^{\Lambda}, U_{0}^{(\nu)}, U_{x}^{(\nu)} \in \widehat{\mathfrak{L}}$ for every $\nu \geq 0$.

Proof. Suppose that the conclusion is valid for every integer up to $\nu$. Using $\kappa t \cdot t^{\Lambda} \Lambda_{x} t^{-\Lambda}=\kappa t \cdot T t^{\Delta} T^{-1} \Lambda_{x} T t^{-\Delta} T^{-1} \in \widehat{\mathfrak{L}}$ and Remark 4.1, we have $U_{1}^{(\nu+1)} \in \widehat{\mathfrak{L}}$. Furthermore, by Remark 4.1,

$$
\begin{aligned}
t^{-\Lambda} U_{1}^{(\nu+1)} t^{\Lambda}=t^{-\Lambda} \mathcal{I} & {\left[-\frac{\kappa t}{1-\kappa t}\left(\left[t^{\Lambda} \Lambda_{x} t^{-\Lambda}, \Lambda_{1}\right]-\left[\Lambda_{1}, t^{\Lambda} U_{x}^{(\nu)} t^{-\Lambda}\right]\right.\right.} \\
& \left.\left.+\left[t^{\Lambda} \Lambda_{x} t^{-\Lambda}, U_{1}^{(\nu)}\right]+\left[t^{\Lambda} U_{x}^{(\nu)} t^{-\Lambda}, U_{1}^{(\nu)}\right]\right)\right] t^{\Lambda} \in \widehat{\mathfrak{L}} .
\end{aligned}
$$

Hence

$$
U_{0}^{(\nu+1)}=-\mathcal{I}\left[\left[t^{-\Lambda} U_{1}^{(\nu+1)} t^{\Lambda}, \Lambda_{0}+U_{0}^{(\nu)}\right]\right] \in \widehat{\mathfrak{L}},
$$

and similarly $U_{x}^{(\nu+1)} \in \widehat{\mathfrak{L}}$. Thus we obtain the proposition.

In the next step we show that, for a suitable domain $D$, the sequence of formal series thus defined is contained in $\mathfrak{L}(D)^{3}$ and converges in it. Let $\Theta_{0}$ be a given positive number, and let $D\left(\varepsilon, \Theta_{0}\right) \subset(\mathbb{C} \backslash\{0\}) \times \mathcal{R}(\mathbb{C} \backslash\{0\})$ be the domain defined by

$$
D\left(\varepsilon, \Theta_{0}\right):=\bigcup_{\kappa \in \mathbb{C} \backslash\{0\}}\{\kappa\} \times\left\{|t|<\varepsilon,\left|\kappa t \cdot t^{-1 / 2}\right|<\varepsilon,|\arg t|<\Theta_{0}\right\} .
$$

Suppose that $\left(\theta_{\iota}\right)$ satisfies (2.4) with $b_{0}>0$. We choose $\varepsilon=\varepsilon\left(\Theta_{0}\right)<2^{-8}$ so small that every $(\kappa, t) \in D\left(\varepsilon, \Theta_{0}\right)$ satisfies $|\log t| \leq|t|^{-1 / 4}$. Note that $|\kappa t|<\varepsilon^{3 / 2}$ for $(\kappa, t) \in D\left(\varepsilon, \Theta_{0}\right)$, and $\sum_{n=0}^{\infty}|\kappa t|^{n}=1 /(1-|\kappa t|)<2$. Since

$$
U_{1}^{(1)}=-\mathcal{I}\left[\frac{\kappa t}{1-\kappa t}\left[t^{\Lambda} \Lambda_{x} t^{-\Lambda}, \Lambda_{1}\right]\right] \in \mathfrak{L}\left(D\left(\varepsilon, \Theta_{0}\right)\right)
$$

by Lemma 4.5 we have $t^{-\Lambda} U_{1}^{(1)} t^{\Lambda} \in \mathfrak{L}\left(D\left(\varepsilon, \Theta_{0}\right)\right)$, so that $U_{0}^{(1)}, U_{x}^{(1)} \in \mathfrak{L}\left(D\left(\varepsilon, \Theta_{0}\right)\right)$. By Lemma 4.4 , in $D\left(\varepsilon, \Theta_{0}\right)$,

$$
\begin{aligned}
& \left\|\kappa t \Lambda_{x}\right\|,\left\|\kappa t \Lambda_{1}\right\|,\left\|\kappa t \cdot t^{\Lambda} \Lambda_{x} t^{-\Lambda}\right\|,\left\|\kappa t \cdot t^{-\Lambda} \Lambda_{1} t^{\Lambda}\right\| \leq \frac{c_{0}}{4}|\kappa t||t|^{-1 / 2} \\
& \left\|U_{1}^{(1)}\right\|,\left\|t^{-\Lambda} U_{1}^{(1)} t^{\Lambda}\right\|,\left\|U_{0}^{(1)}\right\|,\left\|U_{x}^{(1)}\right\| \leq \frac{c_{0}}{4}|\kappa t||t|^{-1 / 2}
\end{aligned}
$$


Here the constant $c_{0}$ may be chosen in such a way that

$$
1+\left\|\Lambda_{0}\right\|+\left\|\Lambda_{x}\right\|+\left\|\Lambda_{1}\right\| \leq c_{0} .
$$

Suppose that, for $1 \leq j \leq \nu$,

$$
U_{1}^{(j)}, t^{-\Lambda} U_{1}^{(j)} t^{\Lambda}, U_{0}^{(j)}, U_{x}^{(j)} \in \mathfrak{L}\left(D\left(\varepsilon, \Theta_{0}\right)\right)
$$

and

$$
\left\|U_{1}^{(j)}\right\|,\left\|t^{-\Lambda} U_{1}^{(j)} t^{\Lambda}\right\|,\left\|U_{0}^{(j)}\right\|,\left\|U_{x}^{(j)}\right\| \leq 2 c_{0}|\kappa t||t|^{-1 / 2},
$$

which are known to be valid for $j=1$. Then, by (5.5) and Lemma 4.5,

$$
\begin{aligned}
& \left\|t^{-\Lambda}\left(U_{1}^{(\nu+1)}-U_{1}^{(\nu)}\right) t^{\Lambda}\right\| \\
= & \| t^{-\Lambda} \mathcal{I}\left[\frac { 1 } { 1 - \kappa t } \left(\kappa t\left[t^{\Lambda}\left(U_{x}^{(\nu)}-U_{x}^{(\nu-1)}\right) t^{-\Lambda}, \Lambda_{1}+U_{1}^{(\nu)}\right]\right.\right. \\
& \left.\left.+\kappa t\left[t^{\Lambda}\left(\Lambda_{x}+U_{x}^{(\nu-1)}\right) t^{-\Lambda}, U_{1}^{(\nu)}-U_{1}^{(\nu-1)}\right]\right)\right] t^{\Lambda} \| \\
\leq & 2 \cdot 2 \cdot 100\|T\|^{3}\left\|T^{-1}\right\|^{3}|\kappa t||t|^{-1 / 2}\left(\left\|U_{x}^{(\nu)}-U_{x}^{(\nu-1)}\right\|\left(\left\|\Lambda_{1}\right\|+\left\|U_{1}^{(\nu)}\right\|\right)\right. \\
& \left.+\left(\left\|\Lambda_{x}\right\|+\left\|U_{x}^{(\nu-1)}\right\|\right)\left\|U_{1}^{(\nu)}-U_{1}^{(\nu-1)}\right\|\right) \\
\leq & 400\|T\|^{3}\left\|T^{-1}\right\|^{3}|\kappa t||t|^{-1 / 2} c_{0}(1+2 \varepsilon)\left(\left\|U_{x}^{(\nu)}-U_{x}^{(\nu-1)}\right\|+\left\|U_{1}^{(\nu)}-U_{1}^{(\nu-1)}\right\|\right) \\
\leq & 500\|T\|^{3}\left\|T^{-1}\right\|^{3} c_{0}|\kappa t||t|^{-1 / 2}\left(\left\|U_{x}^{(\nu)}-U_{x}^{(\nu-1)}\right\|+\left\|U_{1}^{(\nu)}-U_{1}^{(\nu-1)}\right\|\right)
\end{aligned}
$$

in $D\left(\varepsilon, \Theta_{0}\right)$. By Lemma 4.4 ,

$$
\begin{aligned}
\left\|\kappa t \cdot t^{\Lambda}\left(U_{x}^{(\nu)}-U_{x}^{(\nu-1)}\right) t^{-\Lambda}\right\| & \leq 2\|T\|^{2}\left\|T^{-1}\right\|^{2}|\kappa t||t|^{-1 / 2}\left\|U_{x}^{(\nu)}-U_{x}^{(\nu-1)}\right\| \\
\left\|\kappa t \cdot t^{\Lambda} U_{x}^{(\nu-1)} t^{-\Lambda}\right\| & \leq 2\|T\|^{2}\left\|T^{-1}\right\|^{2}|\kappa t||t|^{-1 / 2}\left\|U_{x}^{(\nu-1)}\right\| \\
& \leq\|T\|^{2}\left\|T^{-1}\right\|^{2} c_{0}|\kappa t||t|^{-1 / 2}
\end{aligned}
$$

and hence

$$
\begin{aligned}
& \left\|U_{1}^{(\nu+1)}-U_{1}^{(\nu)}\right\| \\
& \begin{array}{l}
\leq 2 \cdot 2 \cdot 2\left(\left\|\kappa t \cdot t^{\Lambda}\left(U_{x}^{(\nu)}-U_{x}^{(\nu-1)}\right) t^{-\Lambda}\right\|\left(\left\|\Lambda_{1}\right\|+\left\|U_{1}^{(\nu)}\right\|\right)\right. \\
\left.\quad+\left(\left\|\kappa t \cdot t^{\Lambda} \Lambda_{x} t^{-\Lambda}\right\|+\left\|\kappa t \cdot t^{\Lambda} U_{x}^{(\nu-1)} t^{-\Lambda}\right\|\right)\left\|U_{1}^{(\nu)}-U_{1}^{(\nu-1)}\right\|\right) \\
\leq 8\left(2\|T\|^{2}\left\|T^{-1}\right\|^{2}|\kappa t||t|^{-1 / 2}\left\|U_{x}^{(\nu)}-U_{x}^{(\nu-1)}\right\| \cdot\left(c_{0}+2 c_{0} \varepsilon\right)\right. \\
\left.\quad+\left(c_{0} / 4+\|T\|^{2}\left\|T^{-1}\right\|^{2} c_{0}\right)|\kappa t||t|^{-1 / 2} \cdot\left\|U_{1}^{(\nu)}-U_{1}^{(\nu-1)}\right\|\right) \\
\leq 20\|T\|^{2}\left\|T^{-1}\right\|^{2} c_{0}|\kappa t||t|^{-1 / 2}\left(\left\|U_{x}^{(\nu)}-U_{x}^{(\nu-1)}\right\|+\left\|U_{1}^{(\nu)}-U_{1}^{(\nu-1)}\right\|\right) .
\end{array}
\end{aligned}
$$


Using (5.9), we obtain

$$
\begin{aligned}
& \left\|U_{0}^{(\nu+1)}-U_{0}^{(\nu)}\right\| \\
& =\left\|\mathcal{I}\left[\left[t^{-\Lambda}\left(U_{1}^{(\nu+1)}-U_{1}^{(\nu)}\right) t^{\Lambda}, \Lambda_{0}+U_{0}^{(\nu)}\right]+\left[t^{-\Lambda} U_{1}^{(\nu)} t^{\Lambda}, U_{0}^{(\nu)}-U_{0}^{(\nu-1)}\right]\right]\right\| \\
& \leq 4\left(\left\|t^{-\Lambda}\left(U_{1}^{(\nu+1)}-U_{1}^{(\nu)}\right) t^{\Lambda}\right\|\left(\left\|\Lambda_{0}\right\|+\left\|U_{0}^{(\nu)}\right\|\right)+\left\|t^{-\Lambda} U_{1}^{(\nu)} t^{\Lambda}\right\|\left\|U_{0}^{(\nu)}-U_{0}^{(\nu-1)}\right\|\right) \\
& \leq 4\left(500\|T\|^{3}\left\|T^{-1}\right\|^{3} c_{0}\left(c_{0}+2 c_{0} \varepsilon\right)+2 c_{0}\right)|\kappa t||t|^{-1 / 2} \\
& \quad \times\left(\left\|U_{0}^{(\nu)}-U_{0}^{(\nu-1)}\right\|+\left\|U_{x}^{(\nu)}-U_{x}^{(\nu-1)}\right\|+\left\|U_{1}^{(\nu)}-U_{1}^{(\nu-1)}\right\|\right) \\
& \leq 2500\|T\|^{3}\left\|T^{-1}\right\|^{3} c_{0}^{2}|\kappa t||t|^{-1 / 2} \\
& \quad \times\left(\left\|U_{0}^{(\nu)}-U_{0}^{(\nu-1)}\right\|+\left\|U_{x}^{(\nu)}-U_{x}^{(\nu-1)}\right\|+\left\|U_{1}^{(\nu)}-U_{1}^{(\nu-1)}\right\|\right) .
\end{aligned}
$$

Furthermore

$$
\begin{aligned}
\left\|U_{x}^{(\nu+1)}-U_{x}^{(\nu)}\right\| & \\
= & \left\|\mathcal{I}\left[\left[t^{-\Lambda}\left(U_{1}^{(\nu+1)}-U_{1}^{(\nu)}\right) t^{\Lambda}, \Lambda_{x}+U_{x}^{(\nu)}\right]+\left[t^{-\Lambda} U_{1}^{(\nu)} t^{\Lambda}, U_{x}^{(\nu)}-U_{x}^{(\nu-1)}\right]\right]\right\| \\
& +\| \mathcal{I}\left[\frac { \kappa t } { 1 - \kappa t } \left(\left[t^{-\Lambda}\left(U_{1}^{(\nu+1)}-U_{1}^{(\nu)}\right) t^{\Lambda}, \Lambda_{x}+U_{x}^{(\nu)}\right]\right.\right. \\
& \left.\left.+\left[t^{-\Lambda}\left(\Lambda_{1}+U_{1}^{(\nu)}\right) t^{\Lambda}, U_{x}^{(\nu)}-U_{x}^{(\nu-1)}\right]\right)\right] \| \\
\leq & 4\left(\left\|t^{-\Lambda}\left(U_{1}^{(\nu+1)}-U_{1}^{(\nu)}\right) t^{\Lambda}\right\|\left(\left\|\Lambda_{x}\right\|+\left\|U_{x}^{(\nu)}\right\|\right)+\left\|t^{-\Lambda} U_{1}^{(\nu)} t^{\Lambda}\right\|\left\|U_{x}^{(\nu)}-U_{x}^{(\nu-1)}\right\|\right) \\
& +8\left(\left\|t^{-\Lambda}\left(U_{1}^{(\nu+1)}-U_{1}^{(\nu)}\right) t^{\Lambda}\right\|\left(\left\|\kappa t \Lambda_{x}\right\|+|\kappa t|\left\|U_{x}^{(\nu)}\right\|\right)\right. \\
& \left.+\left(\left\|\kappa t \cdot t^{-\Lambda} \Lambda_{1} t^{\Lambda}\right\|+|\kappa t|\left\|t^{-\Lambda} U_{1}^{(\nu)} t^{\Lambda}\right\|\right)\left\|U_{x}^{(\nu)}-U_{x}^{(\nu-1)}\right\|\right) \\
\leq & 4\left\|t^{-\Lambda}\left(U_{1}^{(\nu+1)}-U_{1}^{(\nu)}\right) t^{\Lambda}\right\|\left(c_{0}+2 c_{0} \varepsilon+2\left(c_{0} \varepsilon / 4+2 c_{0} \varepsilon^{2}\right)\right) \\
\leq & 5 c_{0}\left\|t^{-\Lambda}\left(U_{1}^{(\nu+1)}-U_{1}^{(\nu)}\right) t^{\Lambda}\right\|+12 c_{0}|\kappa t||t|^{-1 / 2}\left\|U_{x}^{(\nu)}-U_{x}^{(\nu-1)}\right\| \\
\leq & 2600\|T\|^{3}\left\|T^{-1}\right\|^{3} c_{0}^{2}|\kappa t||t|^{-1 / 2}\left(\left\|U_{x}^{(\nu)}-U_{x}^{(\nu-1)}\right\|+\left\|U_{1}^{(\nu)}-U_{1}^{(\nu-1)}\right\|\right) .
\end{aligned}
$$

In each evaluation above, we have used $|\kappa t||t|^{-1 / 2}<\varepsilon<2^{-8},|\kappa t|<\varepsilon^{3 / 2}<2^{-12}$ and $1 /(1-|\kappa t|)<2$. The constants $\|T\|$ and $\left\|T^{-1}\right\|$ depend only on $\left(\theta_{\iota}\right)$ satisfying (2.4) with $b_{0}>0$. Thus we have the following:

Proposition 5.3. If (5.7) and (5.8) are valid for $1 \leq j \leq \nu$, then $U_{1}^{(\nu+1)}$, $t^{-\Lambda} U_{1}^{(\nu+1)} t^{\Lambda}, U_{0}^{(\nu+1)}, U_{x}^{(\nu+1)}$ are in $\mathfrak{L}\left(D\left(\varepsilon, \Theta_{0}\right)\right)$, and

$$
\begin{aligned}
\left\|U_{1}^{(\nu+1)}-U_{1}^{(\nu)}\right\| & \leq K_{0}|\kappa t||t|^{-1 / 2}\left(\left\|U_{x}^{(\nu)}-U_{x}^{(\nu-1)}\right\|+\left\|U_{1}^{(\nu)}-U_{1}^{(\nu-1)}\right\|\right), \\
\left\|t^{-\Lambda}\left(U_{1}^{(\nu+1)}-U_{1}^{(\nu)}\right) t^{\Lambda}\right\| & \leq K_{0}|\kappa t||t|^{-1 / 2}\left(\left\|U_{x}^{(\nu)}-U_{x}^{(\nu-1)}\right\|+\left\|U_{1}^{(\nu)}-U_{1}^{(\nu-1)}\right\|\right), \\
\left\|U_{0}^{(\nu+1)}-U_{0}^{(\nu)}\right\| \leq K_{0}|\kappa t||t|^{-1 / 2}\left(\left\|U_{x}^{(\nu)}-U_{x}^{(\nu-1)}\right\|+\left\|U_{1}^{(\nu)}-U_{1}^{(\nu-1)}\right\|\right. & \left.+\left\|U_{0}^{(\nu)}-U_{0}^{(\nu-1)}\right\|\right),
\end{aligned}
$$




$$
\left\|U_{x}^{(\nu+1)}-U_{x}^{(\nu)}\right\| \leq K_{0}|\kappa t||t|^{-1 / 2}\left(\left\|U_{x}^{(\nu)}-U_{x}^{(\nu-1)}\right\|+\left\|U_{1}^{(\nu)}-U_{1}^{(\nu-1)}\right\|\right),
$$

where $K_{0}>c_{0}$ is a positive constant depending only on $\Theta_{0}$ and $b_{0}$.

This fact leads us to

Proposition 5.4. For every $\nu \geq 1$,

(i) $U_{1}^{(\nu)}, t^{-\Lambda} U_{1}^{(\nu)} t^{\Lambda}, U_{0}^{(\nu)}, U_{x}^{(\nu)} \in \mathfrak{L}\left(D\left(\varepsilon_{0}, \Theta_{0}\right)\right)$,

(ii) $\left\|U_{1}^{(\nu)}\right\|,\left\|t^{-\Lambda} U_{1}^{(\nu)} t^{\Lambda}\right\|,\left\|U_{0}^{(\nu)}\right\|,\left\|U_{x}^{(\nu)}\right\| \leq 2 c_{0}|\kappa t||t|^{-1 / 2}$,

(iii) $\left\|U_{\iota}^{(\nu)}-U_{\iota}^{(\nu-1)}\right\| \leq\left(4 K_{0}|\kappa t||t|^{-1 / 2}\right)^{\nu}$ for $\iota=0, x, 1$,

(iv) $\left\|t^{-\Lambda}\left(U_{1}^{(\nu)}-U_{1}^{(\nu-1)}\right) t^{\Lambda}\right\| \leq\left(4 K_{0}|\kappa t||t|^{-1 / 2}\right)^{\nu}$,

where $\varepsilon_{0}$ is a sufficiently small positive number depending only on $\Theta_{0}$ and $b_{0}$.

Proof. We verify assertions (i) through (iv) by induction on $\nu$; they are valid for $\nu=1$ by (5.6). Suppose that they are valid for every integer up to $\nu$. Set

$$
u^{(j)}:=\sum_{\iota=0, x, 1}\left\|U_{\iota}^{(j)}-U_{\iota}^{(j-1)}\right\|+\left\|t^{-\Lambda}\left(U_{1}^{(j)}-U_{1}^{(j-1)}\right) t^{\Lambda}\right\|
$$

for $j \geq 1$. Then, by Proposition 5.3 and (5.6), $u^{(1)} \leq c_{0}|\kappa t||t|^{-1 / 2}$ and $u^{(j+1)} \leq$ $4 K_{0}|\kappa t||t|^{-1 / 2} u^{(j)}$ for $1 \leq j \leq \nu$. Hence

$$
u^{(j+1)} \leq\left(4 K_{0}|\kappa t||t|^{-1 / 2}\right)^{j} u^{(1)} \leq\left(4 K_{0}|\kappa t||t|^{-1 / 2}\right)^{j+1}
$$

for $1 \leq j \leq \nu$, and we have

$$
\begin{aligned}
& \left\|U_{0}^{(\nu+1)}\right\|+\left\|U_{x}^{(\nu+1)}\right\|+\left\|U_{1}^{(\nu+1)}\right\|+\left\|t^{-\Lambda} U_{1}^{(\nu+1)} t^{\Lambda}\right\| \\
& \quad \leq \sum_{j=1}^{\nu+1} u^{(j)} \leq \sum_{j=0}^{\nu}\left(4 K_{0}|\kappa t||t|^{-1 / 2}\right)^{j} u^{(1)} \leq \frac{c_{0}|\kappa t||t|^{-1 / 2}}{1-4 K_{0} \varepsilon_{0}} \leq 2 c_{0}|\kappa t||t|^{-1 / 2}
\end{aligned}
$$

for $(\kappa, t) \in D\left(\varepsilon_{0}, \Theta_{0}\right)$, provided that $1-4 K_{0} \varepsilon_{0}>1 / 2$. Thus (i) and (ii) are valid for $\nu+1$, and (5.10) with $j=\nu$ implies (iii) and (iv) for $\nu+1$. This completes the proof.

Remark 5.1. By (iii) and (iv) of Proposition 5.4, the coefficients of the terms $(\kappa t)^{n} \log ^{m} t$ in $U_{\iota}^{(\nu)}$ or $t^{-\Lambda} U_{1}^{(\nu)} t^{\Lambda}$ are fixed if $\nu \geq n$.

By Proposition 5.4, $\lim _{\nu \rightarrow \infty} U_{\iota}^{(\nu)}(\iota=0, x, 1)$ and $\lim _{\nu \rightarrow \infty} t^{-\Lambda} U_{1}^{(\nu)} t^{\Lambda}$ exist and belong to $\mathfrak{L}\left(D\left(\varepsilon_{0}, \Theta_{0}\right)\right)$. Moreover these series satisfy the relations in (5.4). Thus we have

Proposition 5.5. The triple $\left(U_{0}^{\infty}, U_{x}^{\infty}, U_{1}^{\infty}\right)=\lim _{\nu \rightarrow \infty}\left(U_{0}^{(\nu)}, U_{x}^{(\nu)}, U_{1}^{(\nu)}\right)$ satisfies $U_{0}^{\infty}, U_{x}^{\infty}, U_{1}^{\infty}, t^{-\Lambda} U_{1}^{\infty} t^{\Lambda} \in \mathfrak{L}\left(D\left(\varepsilon_{0}, \Theta_{0}\right)\right)$ and solves system $(5.2)$. 
Recall the subring $\widehat{\mathfrak{L}}^{*} \subset \widehat{\mathfrak{L}}$ consisting of $\sum_{n=1}^{\infty} \sum_{m=0}^{n} C_{m}^{n}(\kappa t)^{n} \log ^{m} t$ with $C_{m}^{n} \in M_{2}\left(\mathbb{C}_{\theta}\right)$.

Proposition 5.6. If $\Lambda=\Lambda_{0}+\Lambda_{x}$ and $T$ satisfy (P.2') and if $\left(T^{-1} \Lambda_{0} T\right)_{21}=0$, then $\kappa t \cdot t^{\Lambda} U_{0}^{\infty} t^{-\Lambda}, \kappa t \cdot t^{\Lambda} U_{x}^{\infty} t^{-\Lambda}, U_{1}^{\infty} \in \widehat{\mathfrak{L}}^{*}$.

Proof. Write (5.5) in the form

$$
\begin{aligned}
U_{1}^{(0)}= & Z_{0}^{(0)}=Z_{x}^{(0)} \equiv 0, \\
U_{1}^{(\nu+1)}= & -\mathcal{I}\left[\frac{\kappa t}{1-\kappa t}\left[t^{\Lambda} \Lambda_{x} t^{-\Lambda}+Z_{x}^{(\nu)}, \Lambda_{1}+U_{1}^{(\nu)}\right]\right], \\
\kappa t Z_{0}^{(\nu+1)}= & -\kappa t \cdot t^{\Lambda} \mathcal{I}\left[t^{-\Lambda}\left[U_{1}^{(\nu+1)}, t^{\Lambda} \Lambda_{0} t^{-\Lambda}+Z_{0}^{(\nu)}\right] t^{\Lambda}\right] t^{-\Lambda}, \\
\kappa t Z_{x}^{(\nu+1)}= & -\kappa t \cdot t^{\Lambda} \mathcal{I}\left[t^{-\Lambda}\left[U_{1}^{(\nu+1)}, t^{\Lambda} \Lambda_{x} t^{-\Lambda}+Z_{x}^{(\nu)}\right] t^{\Lambda}\right] t^{-\Lambda} \\
& -\kappa t \cdot t^{\Lambda} \mathcal{I}\left[t^{-\Lambda} \frac{\kappa t}{1-\kappa t}\left[\Lambda_{1}+U_{1}^{(\nu+1)}, t^{\Lambda} \Lambda_{x} t^{-\Lambda}+Z_{x}^{(\nu)}\right] t^{\Lambda}\right] t^{-\Lambda},
\end{aligned}
$$

where $Z_{0}^{(\nu)}=t^{\Lambda} U_{0}^{(\nu)} t^{-\Lambda}, Z_{x}^{(\nu)}=t^{\Lambda} U_{x}^{(\nu)} t^{-\Lambda}$. If $\left(T^{-1} \Lambda_{0} T\right)_{21}=\left(T^{-1} \Lambda_{x} T\right)_{21}=0$, then $\kappa t \cdot t^{\Lambda} \Lambda_{0} t^{-\Lambda}=\kappa t T t^{\Delta} T^{-1} \Lambda_{0} T t^{-\Delta} T^{-1}, \kappa t \cdot t^{\Lambda} \Lambda_{x} t^{-\Lambda} \in \widehat{\mathfrak{L}}^{*}$. Using Lemma 4.6, we can verify by induction on $\nu$ that $U_{1}^{(\nu)}, \kappa t Z_{0}^{(\nu)}, \kappa t Z_{x}^{(\nu)} \in \widehat{\mathfrak{L}}^{*}$ for every $\nu$. This fact combined with Remark 5.1 leads us to the proposition.

5.2.2. Completion of the proof of Theorem 2.2. System (5.2) is derived from (5.1) under the supposition $A_{0}+A_{x}+A_{1} \equiv-\left(\theta_{\infty} / 2\right) J$. We need to verify that

$$
\left(A_{0}^{*}, A_{x}^{*}, A_{1}^{*}\right)=\left(t^{\Lambda}\left(\Lambda_{0}+U_{0}^{\infty}\right) t^{-\Lambda}, t^{\Lambda}\left(\Lambda_{x}+U_{x}^{\infty}\right) t^{-\Lambda}, \Lambda_{1}+U_{1}^{\infty}\right)
$$

with $\left(U_{0}^{\infty}, U_{x}^{\infty}, U_{1}^{\infty}\right)$ obtained above solves (5.1). Set $B_{0}^{*}=\Lambda_{0}+U_{0}^{\infty}=t^{-\Lambda} A_{0}^{*} t^{\Lambda}$ and $B_{x}^{*}=\Lambda_{x}+U_{x}^{\infty}=t^{-\Lambda} A_{x}^{*} t^{\Lambda}$. Since $\left(B_{0}^{*}, B_{x}^{*}, A_{1}^{*}\right)$ satisfies (5.3),

$$
\begin{aligned}
t \frac{d}{d t} & \left(A_{0}^{*}+A_{x}^{*}\right)=t \frac{d}{d t}\left(t^{\Lambda}\left(B_{0}^{*}+B_{x}^{*}\right) t^{-\Lambda}\right)=t^{\Lambda}\left(t \frac{d}{d t}\left(B_{0}^{*}+B_{x}^{*}\right)+\left[\Lambda, B_{0}^{*}+B_{x}^{*}\right]\right) t^{-\Lambda} \\
& =t^{\Lambda}\left(\left[t^{-\Lambda}\left(\Lambda_{1}-A_{1}^{*}\right) t^{\Lambda}, B_{0}^{*}+B_{x}^{*}\right]-\frac{\kappa t}{1-\kappa t}\left[t^{-\Lambda} A_{1}^{*} t^{\Lambda}, B_{x}^{*}\right]+\left[\Lambda, B_{0}^{*}+B_{x}^{*}\right]\right) t^{-\Lambda} \\
& =\left[\Lambda_{1}-A_{1}^{*}, A_{0}^{*}+A_{x}^{*}\right]-\frac{\kappa t}{1-\kappa t}\left[A_{1}^{*}, t^{\Lambda} B_{x}^{*} t^{-\Lambda}\right]+\left[\Lambda, A_{0}^{*}+A_{x}^{*}\right] \\
& =\left[\Lambda+\Lambda_{1}-A_{1}^{*}, A_{0}^{*}+A_{x}^{*}\right]-t \frac{d A_{1}^{*}}{d t}
\end{aligned}
$$

that is,

$$
t \frac{d}{d t}\left(A_{0}^{*}+A_{x}^{*}+A_{1}^{*}\right)=\left[\Lambda+\Lambda_{1}-A_{1}^{*}, A_{0}^{*}+A_{x}^{*}\right] .
$$


Set $X:=A_{0}^{*}+A_{x}^{*}+A_{1}^{*}+\left(\theta_{\infty} / 2\right) J$. Then, by (P.3),

$$
t \frac{d X}{d t}=\left[\Lambda+\Lambda_{1}-A_{1}^{*}, X-A_{1}^{*}+\Lambda_{1}+\Lambda\right]=\left[\Lambda+\Lambda_{1}-A_{1}^{*}, X\right]=\left[\Lambda-U_{1}^{\infty}, X\right],
$$

which can be written in the form

$$
t \frac{d}{d t}\left(t^{-\Lambda} X t^{\Lambda}\right)=-\left[t^{-\Lambda} U_{1}^{\infty} t^{\Lambda}, t^{-\Lambda} X t^{\Lambda}\right]
$$

Recall that $t^{-\Lambda} U_{1}^{\infty} t^{\Lambda} \in \mathfrak{L}\left(D\left(\varepsilon_{0}, \Theta_{0}\right)\right)$ and note that

$t^{-\Lambda} X t^{\Lambda}=t^{-\Lambda}\left(A_{0}^{*}+A_{x}^{*}+A_{1}^{*}-\Lambda_{1}\right) t^{\Lambda}-\Lambda=U_{0}^{\infty}+U_{x}^{\infty}+t^{-\Lambda} U_{1}^{\infty} t^{\Lambda} \in \mathfrak{L}\left(D\left(\varepsilon_{0}, \Theta_{0}\right)\right)$.

From (5.12) it follows that

$$
t \frac{d}{d t}\left(t^{-\Lambda} X t^{\Lambda}+\mathcal{I}\left[\left[t^{-\Lambda} U_{1}^{\infty} t^{\Lambda}, t^{-\Lambda} X t^{\Lambda}\right]\right]\right)=0,
$$

which implies

$$
t^{-\Lambda} X t^{\Lambda}+\mathcal{I}\left[\left[t^{-\Lambda} U_{1}^{\infty} t^{\Lambda}, t^{-\Lambda} X t^{\Lambda}\right]\right] \equiv C \in M_{2}\left(\mathbb{C}_{\theta}\right) .
$$

Since the left-hand member belongs to $\mathfrak{L}\left(D\left(\varepsilon_{0}, \Theta_{0}\right)\right)$, we have $C \equiv 0$, so that

$$
t^{-\Lambda} X t^{\Lambda}=-\mathcal{I}\left[\left[t^{-\Lambda} U_{1}^{\infty} t^{\Lambda}, t^{-\Lambda} X t^{\Lambda}\right]\right]
$$

By Proposition 5.4, $\left\|t^{-\Lambda} U_{1}^{\infty} t^{\Lambda}\right\| \leq 2 c_{0}|\kappa t||t|^{-1 / 2}$. Observing that

$$
\begin{aligned}
\left\|t^{-\Lambda} X t^{\Lambda}\right\| & =\left\|\mathcal{I}\left[\left[t^{-\Lambda} U_{1}^{\infty} t^{\Lambda}, t^{-\Lambda} X t^{\Lambda}\right]\right]\right\| \\
& \leq 4\left\|t^{-\Lambda} U_{1}^{\infty} t^{\Lambda}\right\|\left\|t^{-\Lambda} X t^{\Lambda}\right\| \leq 8 c_{0}|\kappa t||t|^{-1 / 2}\left\|t^{-\Lambda} X t^{\Lambda}\right\|,
\end{aligned}
$$

we deduce $X=A_{0}^{*}+A_{x}^{*}+A_{1}^{*}+\left(\theta_{\infty} / 2\right) J \equiv 0$. Then

$$
\begin{aligned}
t \frac{d A_{0}^{*}}{d t} & =t \frac{d}{d t}\left(t^{\Lambda} B_{0}^{*} t^{-\Lambda}\right)=t^{\Lambda}\left(t \frac{d B_{0}^{*}}{d t}+\left[\Lambda, B_{0}^{*}\right]\right) t^{-\Lambda} \\
& =t^{\Lambda}\left(\left[t^{-\Lambda}\left(\Lambda_{1}-A_{1}^{*}\right) t^{\Lambda}, B_{0}^{*}\right]+\left[\Lambda, B_{0}^{*}\right]\right) t^{-\Lambda} \\
& =\left[\Lambda_{1}+\Lambda-A_{1}^{*}, t^{\Lambda} B_{0}^{*} t^{-\Lambda}\right]=\left[A_{0}^{*}+A_{x}^{*}, A_{0}^{*}\right]=\left[A_{x}^{*}, A_{0}^{*}\right]
\end{aligned}
$$

which means that (5.11) satisfies the first equation of system (5.1). Similarly for the remaining equations of (5.1) we may check the corresponding facts.

We substitute $\kappa t=x$ and $t=\rho x$ into (5.11) to obtain a family of solutions as in Theorem 2.2. If $\left(T^{-1} \Lambda_{0} T\right)_{21}=0$, then $t^{\Lambda} \Lambda_{\iota} t^{-\Lambda}=\Lambda_{\iota}+\left[T \Delta T^{-1}, \Lambda_{\iota}\right] \log t$ $(\iota=0, x)$, and, by Proposition 5.6, $\kappa t\left(A_{0}^{*}-t^{\Lambda} \Lambda_{0} t^{-\Lambda}\right), \kappa t\left(A_{x}^{*}-t^{\Lambda} \Lambda_{x} t^{-\Lambda}\right), A_{1}^{*}-\Lambda_{1}$ are in $\widehat{\mathfrak{L}}^{*}$. Hence $\operatorname{deg} e^{\Lambda \xi} \Lambda_{\iota} e^{-\Lambda \xi} \leq 1, \operatorname{deg} e^{\Lambda \xi} \Pi_{\iota}^{* n}(\xi) e^{-\Lambda \xi} \leq n+1(\iota=0, x)$ and $\operatorname{deg} \Pi_{1}^{* n}(\xi) \leq n$. This completes the proof of Theorem 2.2. 


\section{§5.3. Proof of Theorem 2.1}

Let $\Lambda_{0}, \Lambda_{x}, \Lambda_{1} \in M_{2}\left(\mathbb{C}_{\theta}\left[\sigma, \sigma^{-1}\right]\right), T \in G L_{2}\left(\mathbb{C}_{\theta}\left[\sigma, \sigma^{-1}\right]\right)$ and $\Lambda=\Lambda_{0}+\Lambda_{x}$ satisfy (P.1) through (P.3). To prove Theorem 2.1(1), we construct a solution of (5.2) corresponding to these matrices. Let $\left\{\left(U_{0}^{(\nu)}, U_{x}^{(\nu)}, U_{1}^{(\nu)}\right) \in(\widehat{\mathfrak{S}})^{3} ; \nu \geq 0\right\}$ be the sequence given by a recursive system of the form (5.5) with the operator $\mathcal{I}[\cdot]$ defined in Section 4.2. Then it has the following property, which is verified by using the formal series versions of Lemmas 4.10 and 4.11 (cf. Remark 4.3).

Proposition 5.7. $U_{1}^{(\nu)}, t^{-\Lambda} U_{1}^{(\nu)} t^{\Lambda}, U_{0}^{(\nu)}, U_{x}^{(\nu)} \in \widehat{\mathfrak{S}}$ for every $\nu \geq 0$.

Let $\Sigma_{0}$ be as in Theorem 2.1 and suppose that $\left(\theta_{\iota}\right)$ satisfies (2.4) with $b_{0}>0$. We would like to show the convergence of the sequence in a domain $D\left(\Sigma_{0}, \varepsilon\right) \subset$ $\Sigma_{0} \times(\mathbb{C} \backslash\{0\}) \times \mathcal{R}(\mathbb{C} \backslash\{0\})$ of the form

$$
D\left(\Sigma_{0}, \varepsilon\right):=\bigcup_{(\sigma, \kappa) \in \Sigma_{0} \times(\mathbb{C} \backslash\{0\})}\{(\sigma, \kappa)\} \times\left\{|\kappa t|<\varepsilon,|\kappa t|\left|t^{\sigma}\right|<\varepsilon,|\kappa t|\left|t^{-\sigma}\right|<\varepsilon\right\} .
$$

Using Lemma 4.11, we may show

$$
U_{1}^{(1)}, t^{-\Lambda} U_{1}^{(1)} t^{\Lambda}, U_{0}^{(1)}, U_{x}^{(1)} \in \mathfrak{S}\left(D\left(\Sigma_{0}, \varepsilon\right)\right),
$$

and, for $(\sigma, \kappa, t) \in D\left(\Sigma_{0}, \varepsilon\right)$,

$$
\begin{aligned}
& \left\|\kappa t \Lambda_{x}\right\|,\left\|\kappa t \Lambda_{1}\right\|,\left\|\kappa t \cdot t^{\Lambda} \Lambda_{x} t^{-\Lambda}\right\|,\left\|\kappa t \cdot t^{-\Lambda} \Lambda_{1} t^{\Lambda}\right\| \leq \frac{\tilde{c}_{0}}{4}|\kappa t|\left(\left|t^{\sigma}\right|+\left|t^{-\sigma}\right|\right), \\
& \left\|U_{1}^{(1)}\right\|,\left\|t^{-\Lambda} U_{1}^{(1)} t^{\Lambda}\right\|,\left\|U_{0}^{(1)}\right\|,\left\|U_{x}^{(1)}\right\| \leq \frac{\tilde{c}_{0}}{4}|\kappa t|\left(\left|t^{\sigma}\right|+\left|t^{-\sigma}\right|\right),
\end{aligned}
$$

where $\tilde{c}_{0} \geq 1+\left\|\Lambda_{0}\right\|+\left\|\Lambda_{x}\right\|+\left\|\Lambda_{1}\right\|$ is a constant depending only on $\Sigma_{0}$ and $b_{0}$. Arguments parallel to those in Section 5.2.1 by the use of the lemmas of Section 4.2 lead us to

Proposition 5.8. For every $\nu \geq 1$,

(i) $U_{1}^{(\nu)}, t^{-\Lambda} U_{1}^{(\nu)} t^{\Lambda}, U_{0}^{(\nu)}, U_{x}^{(\nu)} \in \mathfrak{S}\left(D\left(\Sigma_{0}, \varepsilon_{0}\right)\right)$,

(ii) $\left\|U_{1}^{(\nu)}\right\|,\left\|t^{-\Lambda} U_{1}^{(\nu)} t^{\Lambda}\right\|,\left\|U_{0}^{(\nu)}\right\|,\left\|U_{x}^{(\nu)}\right\| \leq 2 \tilde{c}_{0}|\kappa t|\left(\left|t^{\sigma}\right|+\left|t^{-\sigma}\right|\right)$,

(iii) $\left\|U_{\iota}^{(\nu)}-U_{\iota}^{(\nu-1)}\right\| \leq\left(\tilde{K}_{0}|\kappa t|\left(\left|t^{\sigma}\right|+\left|t^{-\sigma}\right|\right)\right)^{\nu}$ for $\iota=0, x, 1$,

(iv) $\left\|t^{-\Lambda}\left(U_{1}^{(\nu)}-U_{1}^{(\nu-1)}\right) t^{\Lambda}\right\| \leq\left(\tilde{K}_{0}|\kappa t|\left(\left|t^{\sigma}\right|+\left|t^{-\sigma}\right|\right)\right)^{\nu}$,

where $\tilde{K}_{0}>\tilde{c}_{0}$ and $\varepsilon_{0}$ are constants depending only on $\Sigma_{0}$ and $b_{0}$.

This yields the solution $\left(U_{0}^{\infty}, U_{x}^{\infty}, U_{1}^{\infty}\right)=\lim _{\nu \rightarrow \infty}\left(U_{0}^{(\nu)}, U_{x}^{(\nu)}, U_{1}^{(\nu)}\right)$ of $(5.2)$ such that $U_{0}^{\infty}, U_{x}^{\infty}, U_{1}^{\infty}, t^{-\Lambda} U_{1}^{\infty} t^{\Lambda} \in \mathfrak{S}\left(D\left(\Sigma_{0}, \varepsilon_{0}\right)\right)$. In the corresponding solution of (5.1) we set $\kappa t=x$ and $t^{\sigma}=\rho x^{\sigma}$ to obtain a family of solutions as in Theorem 2.1(1). 
To show the second half of Theorem 2.1, we consider $\left(U_{0}^{(\nu)}, U_{x}^{(\nu)}, U_{1}^{(\nu)}\right)$ given by the recursive system of the form (5.5) with $\Lambda_{0}, \Lambda_{x}, \Lambda_{1}, \Lambda \in M_{2}\left(\mathbb{C}_{\theta}\left[\sigma_{0}, \sigma_{0}^{-1}\right]\right)$ for $\sigma_{0} \in \Sigma_{+}$. Then $Z_{0}^{(\nu)}=t^{\Lambda} U_{0}^{(\nu)} t^{-\Lambda}, Z_{x}^{(\nu)}=t^{\Lambda} U_{x}^{(\nu)} t^{-\Lambda}$ and $U_{1}^{(\nu)}$ satisfy

$$
\begin{aligned}
U_{1}^{(0)}= & Z_{0}^{(0)}=Z_{x}^{(0)} \equiv 0, \\
U_{1}^{(\nu+1)}= & -\mathcal{I}\left[\frac{\kappa t}{1-\kappa t}\left[t^{\Lambda} \Lambda_{x} t^{-\Lambda}+Z_{x}^{(\nu)}, \Lambda_{1}+U_{1}^{(\nu)}\right]\right], \\
\kappa t Z_{0}^{(\nu+1)}= & -\kappa t \cdot t^{\Lambda} \mathcal{I}\left[t^{-\Lambda}\left[U_{1}^{(\nu+1)}, t^{\Lambda} \Lambda_{0} t^{-\Lambda}+Z_{0}^{(\nu)}\right] t^{\Lambda}\right] t^{-\Lambda}, \\
\kappa t Z_{x}^{(\nu+1)}= & -\kappa t \cdot t^{\Lambda} \mathcal{I}\left[t^{-\Lambda}\left[U_{1}^{(\nu+1)}, t^{\Lambda} \Lambda_{x} t^{-\Lambda}+Z_{x}^{(\nu)}\right] t^{\Lambda}\right] t^{-\Lambda} \\
& -\kappa t \cdot t^{\Lambda} \mathcal{I}\left[t^{-\Lambda} \frac{\kappa t}{1-\kappa t}\left[\Lambda_{1}+U_{1}^{(\nu+1)}, t^{\Lambda} \Lambda_{x} t^{-\Lambda}+Z_{x}^{(\nu)}\right] t^{\Lambda}\right] t^{-\Lambda} .
\end{aligned}
$$

By the supposition $\left(T^{-1} \Lambda_{0} T\right)_{21}=0$, the matrices $t^{\Lambda} \Lambda_{0} t^{-\Lambda}$ and $t^{\Lambda} \Lambda_{x} t^{-\Lambda}$ with $\Lambda=T\left(\sigma_{0} / 2\right) J T^{-1}$ do not contain the term $t^{-\sigma_{0}}$, so $\kappa t \cdot t^{\Lambda} \Lambda_{0} t^{-\Lambda}, \kappa t \cdot t^{\Lambda} \Lambda_{x} t^{-\Lambda}$ are in $\widehat{\mathfrak{S}}^{+}\left(\sigma_{0}\right)$.

Let us consider the case where $\sigma_{0} \in \Sigma_{+} \cap\{\operatorname{Re} \sigma>1\}$. For such $\sigma_{0}$, we show the convergence of the sequence $\left\{\left(\kappa t Z_{0}^{(\nu)}, \kappa t Z_{x}^{(\nu)}, U_{1}^{(\nu)}\right)\right\}$. To do so, set

$$
D^{+}\left(\sigma_{0}, \varepsilon\right):=\bigcup_{\kappa \in \mathbb{C} \backslash\{0\}}\{\kappa\} \times\left\{|\kappa t|<\varepsilon,|\kappa t|\left|t^{\sigma_{0}}\right|<\varepsilon\right\} \subset(\mathbb{C} \backslash\{0\}) \times \mathcal{R}(\mathbb{C} \backslash\{0\}) .
$$

By Lemma $4.13, U_{1}^{(1)}, \kappa t Z_{0}^{(1)}, \kappa t Z_{x}^{(1)} \in \mathfrak{S}^{+}\left(D^{+}\left(\sigma_{0}, \varepsilon\right)\right)$, and for $(\kappa, t) \in D^{+}\left(\sigma_{0}, \varepsilon\right)$,

$$
\begin{aligned}
& \left\|\kappa t \Lambda_{1}\right\|,\left\|\kappa t \cdot t^{\Lambda} \Lambda_{0} t^{-\Lambda}\right\|,\left\|\kappa t \cdot t^{\Lambda} \Lambda_{x} t^{-\Lambda}\right\| \leq \frac{c_{0}^{+}}{4}|\kappa t|\left(1+\left|t^{\sigma_{0}}\right|\right), \\
& \left\|U_{1}^{(1)}\right\|,\left\|\kappa t Z_{0}^{(1)}\right\|,\left\|\kappa t Z_{x}^{(1)}\right\| \leq \frac{c_{0}^{+}}{4}|\kappa t|\left(1+\left|t^{\sigma_{0}}\right|\right),
\end{aligned}
$$

where $c_{0}^{+} \geq 1+\left\|\Lambda_{1}\right\|$ is a constant depending only on $\sigma_{0}$ and $b_{0}$. We suppose, for $j \leq \nu$,

$$
\begin{aligned}
& U_{1}^{(j)}, \kappa t Z_{0}^{(j)}, \kappa t Z_{x}^{(j)} \in \mathfrak{S}^{+}\left(D^{+}\left(\sigma_{0}, \varepsilon\right)\right), \\
& \left\|U_{1}^{(j)}\right\|,\left\|\kappa t Z_{0}^{(j)}\right\|,\left\|\kappa t Z_{x}^{(j)}\right\| \leq 2 c_{0}^{+}|\kappa t|\left(1+\left|t^{\sigma_{0}}\right|\right)
\end{aligned}
$$

with the convention $U_{1}^{(j)}=\kappa t Z_{0}^{(j)}=\kappa t Z_{x}^{(j)}=0$ for $j \leq 0$. These are valid for $j \leq 1$. The recursive relations above give

$$
\begin{aligned}
U_{1}^{(\nu+1)}-U_{1}^{(\nu)}=-\mathcal{I}\left[\frac { \kappa t } { 1 - \kappa t } \left(\left[t^{\Lambda} \Lambda_{x} t^{-\Lambda}+Z_{x}^{(\nu)}, U_{1}^{(\nu)}-U_{1}^{(\nu-1)}\right]\right.\right. & \\
& \left.\left.+\left[Z_{x}^{(\nu)}-Z_{x}^{(\nu-1)}, \Lambda_{1}+U_{1}^{(\nu-1)}\right]\right)\right]
\end{aligned}
$$




$$
\begin{aligned}
\kappa t\left(Z_{0}^{(\nu+1)}-Z_{0}^{(\nu)}\right)=-\kappa t \cdot t^{\Lambda} \mathcal{I}\left[t ^ { - \Lambda } \left(\left[U_{1}^{(\nu+1)}-U_{1}^{(\nu)},\right.\right.\right. & \left.t^{\Lambda} \Lambda_{0} t^{-\Lambda}+Z_{0}^{(\nu)}\right] \\
& \left.\left.+\left[U_{1}^{(\nu)}, Z_{0}^{(\nu)}-Z_{0}^{(\nu-1)}\right]\right) t^{\Lambda}\right] t^{-\Lambda}, \\
\kappa t\left(Z_{x}^{(\nu+1)}-Z_{x}^{(\nu)}\right)=-\kappa t \cdot t^{\Lambda} \mathcal{I}\left[t ^ { - \Lambda } \left(\left[U_{1}^{(\nu+1)}-U_{1}^{(\nu)},\right.\right.\right. & \left.t^{\Lambda} \Lambda_{x} t^{-\Lambda}+Z_{x}^{(\nu)}\right] \\
& \left.\left.+\left[U_{1}^{(\nu)}, Z_{x}^{(\nu)}-Z_{x}^{(\nu-1)}\right]\right) t^{\Lambda}\right] t^{-\Lambda} \\
& -\kappa t \cdot t^{\Lambda} \mathcal{I}\left[t ^ { - \Lambda } \frac { \kappa t } { 1 - \kappa t } \left(\left[U_{1}^{(\nu+1)}-U_{1}^{(\nu)}, t^{\Lambda} \Lambda_{x} t^{-\Lambda}+Z_{x}^{(\nu)}\right]\right.\right. \\
& \left.\left.+\left[U_{1}^{(\nu)}, Z_{x}^{(\nu)}-Z_{x}^{(\nu-1)}\right]\right) t^{\Lambda}\right] t^{-\Lambda} .
\end{aligned}
$$

Using Proposition 4.12, we derive

$$
\begin{array}{r}
\left\|U_{1}^{(\nu+1)}-U_{1}^{(\nu)}\right\| \leq 4 L_{0}^{+}\left(\left\|\kappa t\left(t^{\Lambda} \Lambda_{x} t^{-\Lambda}+Z_{x}^{(\nu)}\right)\right\|\left\|U_{1}^{(\nu)}-U_{1}^{(\nu-1)}\right\|\right. \\
\left.+\left(\left\|\Lambda_{1}\right\|+\left\|U_{1}^{(\nu-1)}\right\|\right)\left\|\kappa t\left(Z_{x}^{(\nu)}-Z_{x}^{(\nu-1)}\right)\right\|\right) \\
\leq 12 L_{0}^{+} c_{0}^{+}\left(|\kappa t|\left(1+\left|t^{\sigma_{0}}\right|\right)\left\|U_{1}^{(\nu)}-U_{1}^{(\nu-1)}\right\|+\left\|\kappa t\left(Z_{x}^{(\nu)}-Z_{x}^{(\nu-1)}\right)\right\|\right)
\end{array}
$$

if $\varepsilon<1 / 2$. Furthermore, by Lemma 4.13,

$$
\begin{aligned}
& \left\|\kappa t\left(Z_{0}^{(\nu+1)}-Z_{0}^{(\nu)}\right)\right\| \leq 2 \tilde{L}_{0}^{+}\|T\|^{3}\left\|T^{-1}\right\|^{3} \\
& \times\left(\left\|\kappa t\left(t^{\Lambda} \Lambda_{0} t^{-\Lambda}+Z_{0}^{(\nu)}\right)\right\|\left\|U_{1}^{(\nu+1)}-U_{1}^{(\nu)}\right\|+\left\|U_{1}^{(\nu)}\right\|\left\|\kappa t\left(Z_{0}^{(\nu)}-Z_{0}^{(\nu-1)}\right)\right\|\right) \\
& \leq 6 \tilde{L}_{0}^{+}\|T\|^{3}\left\|T^{-1}\right\|^{3} c_{0}^{+}|\kappa t|\left(1+\left|t^{\sigma_{0}}\right|\right)\left(\left\|U_{1}^{(\nu+1)}-U_{1}^{(\nu)}\right\|+\left\|\kappa t\left(Z_{0}^{(\nu)}-Z_{0}^{(\nu-1)}\right)\right\|\right),
\end{aligned}
$$

and

$$
\begin{aligned}
\| \kappa t\left(Z_{x}^{(\nu+1)}-\right. & \left.Z_{x}^{(\nu)}\right)\left\|\leq 20 \tilde{L}_{0}^{+}\right\| T\left\|^{3}\right\| T^{-1} \|^{3} c_{0}^{+} \\
& \times|\kappa t|\left(1+\left|t^{\sigma_{0}}\right|\right)\left(\left\|U_{1}^{(\nu+1)}-U_{1}^{(\nu)}\right\|+\left\|\kappa t\left(Z_{x}^{(\nu)}-Z_{x}^{(\nu-1)}\right)\right\|\right) .
\end{aligned}
$$

In the last inequality we replace $\nu$ by $\nu-1$ to obtain

$$
\begin{aligned}
& \left\|\kappa t\left(Z_{x}^{(\nu)}-Z_{x}^{(\nu-1)}\right)\right\| \\
& \quad \leq K_{1}^{+}|\kappa t|\left(1+\left|t^{\sigma_{0}}\right|\right)\left(\left\|U_{1}^{(\nu)}-U_{1}^{(\nu-1)}\right\|+\left\|\kappa t\left(Z_{x}^{(\nu-1)}-Z_{x}^{(\nu-2)}\right)\right\|\right)
\end{aligned}
$$

with $K_{1}^{+}=20 \tilde{L}_{0}^{+}\|T\|^{3}\left\|T^{-1}\right\|^{3} c_{0}^{+}$. Inequality (5.16) combined with (5.19) gives

$$
\begin{aligned}
\| U_{1}^{(\nu+1)} & -U_{1}^{(\nu)} \| \\
& \leq K_{2}^{+}|\kappa t|\left(1+\left|t^{\sigma_{0}}\right|\right)\left(\left\|U_{1}^{(\nu)}-U_{1}^{(\nu-1)}\right\|+\left\|\kappa t\left(Z_{x}^{(\nu-1)}-Z_{x}^{(\nu-2)}\right)\right\|\right)
\end{aligned}
$$


with some constant $K_{2}^{+} \geq c_{0}^{+}$. Substituting this into (5.17) and (5.18), we have

$$
\begin{aligned}
& \left\|\kappa t\left(Z_{0}^{(\nu+1)}-Z_{0}^{(\nu)}\right)\right\| \leq K_{3}^{+}|\kappa t|\left(1+\left|t^{\sigma_{0}}\right|\right) \\
& \quad \times\left(\left\|U_{1}^{(\nu)}-U_{1}^{(\nu-1)}\right\|+\left\|\kappa t\left(Z_{0}^{(\nu)}-Z_{0}^{(\nu-1)}\right)\right\|+\left\|\kappa t\left(Z_{x}^{(\nu-1)}-Z_{x}^{(\nu-2)}\right)\right\|\right), \\
& \left\|\kappa t\left(Z_{x}^{(\nu+1)}-Z_{x}^{(\nu)}\right)\right\| \leq K_{3}^{+}|\kappa t|\left(1+\left|t^{\sigma_{0}}\right|\right) \\
& \quad \times\left(\left\|U_{1}^{(\nu)}-U_{1}^{(\nu-1)}\right\|+\left\|\kappa t\left(Z_{x}^{(\nu)}-Z_{x}^{(\nu-1)}\right)\right\|+\left\|\kappa t\left(Z_{x}^{(\nu-1)}-Z_{x}^{(\nu-2)}\right)\right\|\right)
\end{aligned}
$$

with some constant $K_{3}^{+} \geq c_{0}^{+}$. Put

$$
\begin{aligned}
v^{(j)}:= & \left\|U_{1}^{(j)}-U_{1}^{(j-1)}\right\| \\
& +\left\|\kappa t\left(Z_{0}^{(j)}-Z_{0}^{(j-1)}\right)\right\|+\left\|\kappa t\left(Z_{x}^{(j)}-Z_{x}^{(j-1)}\right)\right\|+\left\|\kappa t\left(Z_{x}^{(j-1)}-Z_{x}^{(j-2)}\right)\right\| .
\end{aligned}
$$

By (5.13), $v^{(1)} \leq c_{0}^{+}|\kappa t|\left(1+\left|t^{\sigma_{0}}\right|\right)$. From inequalities (5.19) through (5.22) we derive $v^{(j+1)} \leq K_{0}^{+}|\kappa t|\left(1+\left|t^{\sigma_{0}}\right|\right) v^{(j)}$ for every $j \leq \nu$ with some constant $K_{0}^{+} \geq c_{0}^{+}$, which is valid under the suppositions (5.14) and (5.15). By the same argument as in the proof of Proposition 5.4 we have

Proposition 5.9. Under the supposition $\sigma_{0} \in \Sigma_{+} \cap\{\operatorname{Re} \sigma>1\}$, for every $\nu \geq 1$,

(i) $U_{1}^{(\nu)}, \kappa t Z_{0}^{(\nu)}, \kappa t Z_{x}^{(\nu)} \in \mathfrak{S}^{+}\left(D^{+}\left(\sigma_{0}, \varepsilon_{0}\right)\right)$,

(ii) $\left\|U_{1}^{(\nu)}\right\|,\left\|\kappa t Z_{0}^{(\nu)}\right\|,\left\|\kappa t Z_{x}^{(\nu)}\right\| \leq 2 c_{0}^{+}|\kappa t|\left(1+\left|t^{\sigma_{0}}\right|\right)$,

(iii) $\left\|U_{1}^{(\nu)}-U_{1}^{(\nu-1)}\right\|,\left\|\kappa t\left(Z_{\iota}^{(\nu)}-Z_{\iota}^{(\nu-1)}\right)\right\| \leq\left(K_{0}^{+}|\kappa t|\left(1+\left|t^{\sigma_{0}}\right|\right)\right)^{\nu}$ for $\iota=0, x$,

where $K_{0}^{+}>c_{0}^{+}$and $\varepsilon_{0}$ are constants depending only on $\sigma_{0}$ and $b_{0}$.

This proposition implies $\left(U_{1}^{\infty}, \kappa t Z_{0}^{\infty}, \kappa t Z_{x}^{\infty}\right):=\lim _{\nu \rightarrow \infty}\left(U_{1}^{(\nu)}, \kappa t Z_{0}^{(\nu)}, \kappa t Z_{x}^{(\nu)}\right)$ $\in \mathfrak{S}^{+}\left(D^{+}\left(\sigma_{0}, \varepsilon_{0}\right)\right)^{3}$ if $\sigma_{0} \in \Sigma_{+} \cap\{\operatorname{Re} \sigma>1\}$. Denote by $\left(U_{1}^{\infty}(\sigma, t), U_{0}^{\infty}(\sigma, t), U_{x}^{\infty}(\sigma, t)\right)$ the solution of (5.2) for $\sigma \in \Sigma_{0}$ constructed in the proof of Theorem 2.1(1). We may suppose that $\Sigma_{0} \cap\left(\Sigma_{+} \cap\{\operatorname{Re} \sigma>1\}\right) \neq \emptyset$. If $\sigma_{0} \in \Sigma_{0} \cap\left(\Sigma_{+} \cap\{\operatorname{Re} \sigma>1\}\right)$, then

$$
\begin{aligned}
\left(U_{1}^{\infty}\left(\sigma_{0}, t\right), \kappa t \cdot t^{\Lambda} U_{0}^{\infty}\left(\sigma_{0}, t\right) t^{-\Lambda}, \kappa t \cdot t^{\Lambda} U_{x}^{\infty}\left(\sigma_{0}, t\right) t^{-\Lambda}\right) & \\
& \equiv\left(U_{1}^{\infty}, \kappa t Z_{0}^{\infty}, \kappa t Z_{x}^{\infty}\right) \in \mathfrak{S}^{+}\left(D^{+}\left(\sigma_{0}, \varepsilon_{0}\right)\right)^{3}
\end{aligned}
$$

since both triples come from the same sequence defined by the recursive system of the form (5.5). By Lemma 4.10, $\left(U_{1}^{\infty}(\sigma, t), \kappa t \cdot t^{\Lambda} U_{0}^{\infty}(\sigma, t) t^{-\Lambda}, \kappa t \cdot t^{\Lambda} U_{x}^{\infty}(\sigma, t) t^{-\Lambda}\right) \in$ $\mathfrak{S}\left(D\left(\Sigma_{0}, \varepsilon_{0}\right)\right)^{3}$, and we write

$$
\begin{aligned}
U_{1}^{\infty}(\sigma, t) & =\sum_{n=1}^{\infty} \sum_{m=-n}^{n} \Gamma_{1 m}^{n}(\sigma)(\kappa t)^{n} t^{\sigma m}, \\
\kappa t \cdot t^{\Lambda} U_{\iota}^{\infty}(\sigma, t) t^{-\Lambda} & =\sum_{n=1}^{\infty} \sum_{m=-n}^{n} \Gamma_{\iota m}^{n}(\sigma)(\kappa t)^{n} t^{\sigma m} \quad(\iota=0, x)
\end{aligned}
$$


with $\Gamma_{1 m}^{n}(\sigma), \Gamma_{\iota m}^{n}(\sigma) \in M_{2}\left(\mathbb{C}_{\theta}(\sigma)\right)$. Note that $\sigma_{0}$ is algebraic in $\left(\theta_{\iota}\right)$. By $(5.23)$ we find that $\Gamma_{0 m}^{n}\left(\sigma_{0}\right), \Gamma_{x m}^{n}\left(\sigma_{0}\right), \Gamma_{1 m}^{n}\left(\sigma_{0}\right) \equiv 0$ for $-n \leq m<0$ as functions of $\left(\theta_{\iota}\right)$, and $(5.23)$ as a function of $\left(\kappa, t,\left(\theta_{\iota}\right)\right)$ can be continued analytically as long as $\sigma_{0} \in \Sigma_{0} \cup\left(\Sigma_{+} \cap\{\operatorname{Re} \sigma>1\}\right)$. Then

$$
\begin{aligned}
& \left(t^{\Lambda} \Lambda_{0} t^{-\Lambda}+Z_{0}^{\infty}, t^{\Lambda} \Lambda_{x} t^{-\Lambda}+Z_{x}^{\infty}, \Lambda_{1}+U_{1}^{\infty}\right) \\
& \quad=\left(t^{\Lambda}\left(\Lambda_{0}+U_{0}^{\infty}\left(\sigma_{0}, t\right)\right) t^{-\Lambda}, t^{\Lambda}\left(\Lambda_{x}+U_{x}^{\infty}\left(\sigma_{0}, t\right)\right) t^{-\Lambda}, \Lambda_{1}+U_{1}^{\infty}\left(\sigma_{0}, t\right)\right)
\end{aligned}
$$

with $\left(t^{\sigma_{0}}, \kappa t\right)=\left(\rho x^{\sigma_{0}}, x\right)$ solves $(2.1)$ for every $\sigma_{0} \in \Sigma_{0} \cup\left(\Sigma_{+} \cap\{\operatorname{Re} \sigma>1\}\right)$. For each $\sigma_{0} \in \Sigma_{+} \cap\{\operatorname{Re} \sigma \leq 1\}$ we may choose $\Sigma_{0}$ such that $\Sigma_{0} \ni \sigma_{0}$, and hence we obtain the solution as in Theorem 2.1 for $\sigma_{0} \in \Sigma_{+}$.

\section{$\S 6$. Proofs of Theorems 2.3, 2.4 and Corollary 2.5}

For $\sigma \neq 0$, [5, Lemma A.2] with $r=s=1$ gives

Lemma 6.1. The matrices

$$
\begin{aligned}
& \Lambda_{0}=T\left(\begin{array}{cc}
\frac{1}{4 \sigma}\left(\sigma^{2}+\theta_{0}^{2}-\theta_{x}^{2}\right) & 1 \\
\frac{1}{16 \sigma^{2}}\left(\left(\theta_{0}+\theta_{x}\right)^{2}-\sigma^{2}\right)\left(\sigma^{2}-\left(\theta_{0}-\theta_{x}\right)^{2}\right) & \frac{1}{4 \sigma}\left(\theta_{x}^{2}-\theta_{0}^{2}-\sigma^{2}\right)
\end{array}\right) T^{-1} \\
& \Lambda_{x}=T\left(\begin{array}{cc}
\frac{1}{4 \sigma}\left(\sigma^{2}+\theta_{x}^{2}-\theta_{0}^{2}\right) & -1 \\
\frac{1}{16 \sigma^{2}}\left(\left(\theta_{0}+\theta_{x}\right)^{2}-\sigma^{2}\right)\left(\left(\theta_{0}-\theta_{x}\right)^{2}-\sigma^{2}\right) & \frac{1}{4 \sigma}\left(\theta_{0}^{2}-\theta_{x}^{2}-\sigma^{2}\right)
\end{array}\right) T^{-1}, \\
& \Lambda=\left(\begin{array}{cc}
\frac{1}{4 \theta_{\infty}}\left(\theta_{1}^{2}-\theta_{\infty}^{2}-\sigma^{2}\right) & 1 \\
\frac{1}{16 \theta_{\infty}^{2}}\left(\left(\sigma+\theta_{1}\right)^{2}-\theta_{\infty}^{2}\right)\left(\theta_{\infty}^{2}-\left(\sigma-\theta_{1}\right)^{2}\right) & \frac{1}{4 \theta_{\infty}}\left(\sigma^{2}-\theta_{1}^{2}+\theta_{\infty}^{2}\right)
\end{array}\right), \\
& \Lambda_{1}=\left(\begin{array}{cc}
\frac{1}{4 \theta_{\infty}}\left(\sigma^{2}-\theta_{1}^{2}-\theta_{\infty}^{2}\right) & -1 \\
\frac{1}{16 \theta_{\infty}^{2}}\left(\left(\sigma+\theta_{1}\right)^{2}-\theta_{\infty}^{2}\right)\left(\left(\sigma-\theta_{1}\right)^{2}-\theta_{\infty}^{2}\right) & \frac{1}{4 \theta_{\infty}}\left(\theta_{1}^{2}+\theta_{\infty}^{2}-\sigma^{2}\right)
\end{array}\right), \\
& T=\left(\begin{array}{cc}
1 & 1 \\
\frac{1}{4 \theta_{\infty}}\left(\left(\sigma+\theta_{\infty}\right)^{2}-\theta_{1}^{2}\right) & \frac{1}{4 \theta_{\infty}}\left(\left(\sigma-\theta_{\infty}\right)^{2}-\theta_{1}^{2}\right)
\end{array}\right)
\end{aligned}
$$

have the properties (P.1) through (P.3).

Using [6, Proposition 2, Jordan case] with $r=0$ or $-\theta_{0}$, we have

Lemma 6.2. The matrices

$$
\begin{aligned}
\Lambda_{0} & =T\left(\begin{array}{cc} 
\pm \theta_{0} / 2 & 0 \\
\left(\theta_{0}^{2}-\theta_{x}^{2}\right) / 4 & \mp \theta_{0} / 2
\end{array}\right) T^{-1}, \quad \Lambda_{x}=T\left(\begin{array}{cc}
\mp \theta_{0} / 2 & 1 \\
\left(\theta_{x}^{2}-\theta_{0}^{2}\right) / 4 & \pm \theta_{0} / 2
\end{array}\right) T^{-1} \\
\Lambda & =\left(\begin{array}{cc}
\frac{1}{4 \theta_{\infty}}\left(\theta_{1}^{2}-\theta_{\infty}^{2}\right) & 1 \\
-\frac{1}{16 \theta_{\infty}^{2}}\left(\theta_{1}^{2}-\theta_{\infty}^{2}\right)^{2} & \frac{1}{4 \theta_{\infty}}\left(\theta_{\infty}^{2}-\theta_{1}^{2}\right)
\end{array}\right),
\end{aligned}
$$




$$
\begin{aligned}
\Lambda_{1} & =\left(\begin{array}{cc}
-\frac{1}{4 \theta_{\infty}}\left(\theta_{1}^{2}+\theta_{\infty}^{2}\right) & -1 \\
\frac{1}{16 \theta_{\infty}^{2}}\left(\theta_{1}^{2}-\theta_{\infty}^{2}\right)^{2} & \frac{1}{4 \theta_{\infty}}\left(\theta_{1}^{2}+\theta_{\infty}^{2}\right)
\end{array}\right), \\
T & =\left(\begin{array}{cc}
1 & 0 \\
\frac{1}{4 \theta_{\infty}}\left(\theta_{\infty}^{2}-\theta_{1}^{2}\right) & 1
\end{array}\right)
\end{aligned}
$$

have the properties (P.1), (P.2'), (P.3).

Remark 6.1. It is also possible to choose more general matrices

$$
\begin{aligned}
\Lambda & =\left(\begin{array}{cc}
\frac{1}{4 \theta_{\infty}}\left(\theta_{1}^{2}-\theta_{\infty}^{2}\right) & 1-\left(\theta_{1}^{2}-\theta_{\infty}^{2}\right) \tilde{r} \\
-\frac{1}{16 \theta_{\infty}^{2}\left(1-\left(\theta_{1}^{2}-\theta_{\infty}^{2}\right) \tilde{r}\right)}\left(\theta_{1}^{2}-\theta_{\infty}^{2}\right)^{2} & \frac{1}{4 \theta_{\infty}}\left(\theta_{\infty}^{2}-\theta_{1}^{2}\right)
\end{array}\right), \\
\Lambda_{1} & =\left(\begin{array}{cc}
-\frac{1}{4 \theta_{\infty}}\left(\theta_{1}^{2}+\theta_{\infty}^{2}\right) & -1+\left(\theta_{1}^{2}-\theta_{\infty}^{2}\right) \tilde{r} \\
\frac{1}{16 \theta_{\infty}^{2}\left(1-\left(\theta_{1}^{2}-\theta_{\infty}^{2}\right) \tilde{r}\right)}\left(\theta_{1}^{2}-\theta_{\infty}^{2}\right)^{2} & \frac{1}{4 \theta_{\infty}}\left(\theta_{1}^{2}+\theta_{\infty}^{2}\right)
\end{array}\right), \\
T & =\left(\begin{array}{cc}
1 & 4 \theta_{\infty} \tilde{r} \\
\frac{1}{4 \theta_{\infty}\left(1-\left(\theta_{1}^{2}-\theta_{\infty}^{2}\right) \tilde{r}\right)}\left(\theta_{\infty}^{2}-\theta_{1}^{2}\right) & 1
\end{array}\right) .
\end{aligned}
$$

\section{§6.1. Derivation of Theorem 2.3}

Let $\Lambda_{0}, \Lambda_{x}, \Lambda_{1}$ and $T$ be as in Lemma 6.1. For the solution with $\sigma \in \Sigma_{0}$ in Theorem 2.1, $x A_{0}(\sigma, \rho, x), x A_{x}(\sigma, \rho, x), A_{1}(\sigma, \rho, x)-\Lambda_{1}$ can be written in the form $\sum_{n=1}^{\infty} x^{n} \sum_{m=-n}^{n} A_{\iota m}^{n}(\sigma)\left(\rho x^{\sigma}\right)^{m}$ with $A_{\iota m}^{n}(\sigma) \in M_{2}\left(\mathbb{C}_{\theta}(\sigma)\right)(\iota=0, x, 1)$. If we substitute $\left(\rho x^{\sigma}, x\right)=\left(t^{\sigma}, \kappa t\right)$, they become, respectively, $\kappa t \cdot t^{\Lambda}\left(\Lambda_{0}+U_{0}^{\infty}\right) t^{-\Lambda}$, $\kappa t \cdot t^{\Lambda}\left(\Lambda_{x}+U_{x}^{\infty}\right) t^{-\Lambda}, U_{1}^{\infty} \in \mathfrak{S}\left(D\left(\Sigma_{0}, \varepsilon_{0}\right)\right)$ with $U_{0}^{\infty}, U_{x}^{\infty}, U_{1}^{\infty}$ as in the proof of Theorem 2.1 in Section 5.3, and, by Proposition 5.8 and Lemma 4.10,

$$
\left\|\kappa t \cdot t^{\Lambda}\left(\Lambda_{0}+U_{0}^{\infty}\right) t^{-\Lambda}\right\|,\left\|\kappa t \cdot t^{\Lambda}\left(\Lambda_{x}+U_{x}^{\infty}\right) t^{-\Lambda}\right\|,\left\|U_{1}^{\infty}\right\| \leq \tilde{c}_{0}^{*}|\kappa t|\left(\left|t^{\sigma}\right|+\left|t^{-\sigma}\right|\right),
$$

where $\tilde{c}_{0}^{*}$ is a positive constant depending only on $\Sigma_{0}$ and $b_{0}$. Hence, taking $\varepsilon_{0}$ so small that $\varepsilon_{0}<\left(16 \tilde{c}_{0}^{*}\right)^{-1}$ again if necessary, for $(\sigma, \rho, x)$ satisfying

$$
|x|<\varepsilon_{0}, \quad\left|x\left(\rho x^{\sigma}\right)\right|<\varepsilon_{0}, \quad\left|x\left(\rho x^{\sigma}\right)^{-1}\right|<\varepsilon_{0}
$$

we deduce that each entry of $x A_{0}(\sigma, \rho, x), x A_{x}(\sigma, \rho, x)$ and $A_{1}(\sigma, \rho, x)-\Lambda_{1}$ as a double series converges absolutely, and the sum of the absolute values of the summands in each double series does not exceed $1 / 8$. Recall that

$$
y(x)=\frac{x A_{0}(\sigma, \rho, x)_{12}}{x\left(A_{0}(\sigma, \rho, x)+A_{1}(\sigma, \rho, x)\right)_{12}-A_{1}(\sigma, \rho, x)_{12}}
$$

solves (PVI). By the fact mentioned above, the denominator is given by $1-a_{\mathrm{den}}(\sigma, \rho, x)$ with $a_{\mathrm{den}}(\sigma, \rho, x)=\sum_{n=1}^{\infty} x^{n} \sum_{m=-n}^{n} a_{m}^{n}(\sigma)\left(\rho x^{\sigma}\right)^{m}, a_{m}^{n}(\sigma) \in$ 
$\mathbb{C}_{\theta}(\sigma)$ such that $\sum_{n=1}^{\infty}|x|^{n} \sum_{m=-n}^{n}\left|a_{m}^{n}(\sigma)\right|\left|\rho x^{\sigma}\right|^{m}<1 / 2$ for $(\sigma, \rho, x)$ satisfying (6.1). Substituting the series $a_{\mathrm{den}}(\sigma, \rho, x)$ into $\left(1-a_{\mathrm{den}}(\sigma, \rho, x)\right)^{-1}=1+$ $\sum_{j=1}^{\infty} a_{\mathrm{den}}(\sigma, \rho, x)^{j}$ and rearranging the summands, we obtain the product of convergent series

$$
y(x)=x A_{0}(\sigma, \rho, x)_{12}\left(1+\sum_{n=1}^{\infty} x^{n} \sum_{m=-n}^{n} \tilde{a}_{m}^{n}(\sigma)\left(\rho x^{\sigma}\right)^{m}\right) .
$$

Here $\tilde{a}_{m}^{n}(\sigma) \in \mathbb{C}_{\theta}(\sigma)$, because each $a_{\operatorname{den}}(\sigma, \rho, x)^{j}$ consists of summands with $n \geq j$. By Lemma 6.1, the leading term of $x A_{0}(\sigma, \rho, x)$ is

$$
\begin{aligned}
& x\left(\rho x^{\sigma}\right)^{\Lambda / \sigma} \Lambda_{0}\left(\rho x^{\sigma}\right)^{-\Lambda / \sigma}=x T\left(\rho x^{\sigma}\right)^{J / 2} T^{-1} \Lambda_{0} T\left(\rho x^{\sigma}\right)^{-J / 2} T^{-1} \\
& \quad=x T\left(\begin{array}{cc}
\frac{1}{4 \sigma}\left(\sigma^{2}+\theta_{0}^{2}-\theta_{x}^{2}\right) & \rho x^{\sigma} \\
\frac{1}{16 \sigma^{2}}\left(\rho x^{\sigma}\right)^{-1}\left(\left(\theta_{0}+\theta_{x}\right)^{2}-\sigma^{2}\right)\left(\sigma^{2}-\left(\theta_{0}-\theta_{x}\right)^{2}\right) & \frac{1}{4 \sigma}\left(\theta_{x}^{2}-\theta_{0}^{2}-\sigma^{2}\right)
\end{array}\right) T^{-1},
\end{aligned}
$$

whose $(1,2)$-entry is

$x\left(-\frac{\rho x^{\sigma}}{\sigma}+\frac{1}{2 \sigma^{2}}\left(\sigma^{2}+\theta_{0}^{2}-\theta_{x}^{2}\right)-\frac{1}{16 \sigma^{3}}\left(\left(\theta_{0}+\theta_{x}\right)^{2}-\sigma^{2}\right)\left(\left(\theta_{0}-\theta_{x}\right)^{2}-\sigma^{2}\right)\left(\rho x^{\sigma}\right)^{-1}\right)$.

We replace the integration constant $\rho$ by $-\sigma \rho$ to obtain $y(\sigma, \rho, x)$ as in Theorem 2.3 with $\hat{\varepsilon}_{0}=\varepsilon_{0} \inf _{\sigma \in \Sigma_{0}} \min \left\{|\sigma|,|\sigma|^{-1}\right\}>0$. If $\sigma_{0}^{2}=\left(\theta_{0} \pm \theta_{x}\right)^{2}$, then $\left(T^{-1} \Lambda_{0} T\right)_{21}$ vanishes at $\sigma=\sigma_{0}$, and $x A_{0}\left(\sigma_{0}, \rho, x\right), x A_{x}\left(\sigma_{0}, \rho, x\right), A_{1}\left(\sigma_{0}, \rho, x\right)-\Lambda_{1}$ admit representations $\sum_{n=1}^{\infty} x^{n} \sum_{m=0}^{n} A_{\iota m}^{n}\left(\sigma_{0}\right)\left(\rho x^{\sigma_{0}}\right)^{m}(\iota=0, x, 1)$. Hence the second half of the theorem immediately follows.

\section{§6.2. Derivation of Theorem 2.4}

For the solution in Theorem 2.2, $x A_{0}(\rho, x), x A_{x}(\rho, x), A_{1}(\rho, x)-\Lambda_{1}$ can be written in the form $\sum_{n=1}^{\infty} x^{n} \sum_{m=0}^{2 n} A_{\iota m}^{0 n} \log ^{m}(\rho x)$ with $A_{\iota m}^{0 n} \in M_{2}\left(\mathbb{C}_{\theta}\right)(\iota=0, x, 1)$. By Lemma 6.2 the leading term of $x A_{0}(\rho, x)$ is

$$
\begin{aligned}
x(\rho x)^{\Lambda} \Lambda_{0}(\rho x)^{-\Lambda} & =x T(\rho x)^{\Delta} T^{-1} \Lambda_{0} T(\rho x)^{-\Delta} T^{-1} \\
& =x T(\rho x)^{\Delta}\left(\begin{array}{cc} 
\pm \theta_{0} / 2 & 0 \\
\left(\theta_{0}^{2}-\theta_{x}^{2}\right) / 4 & \mp \theta_{0} / 2
\end{array}\right)(\rho x)^{-\Delta} T^{-1},
\end{aligned}
$$

whose $(1,2)$-entry is

$$
x\left(\mp \theta_{0} \log (\rho x)+\frac{1}{4}\left(\theta_{x}^{2}-\theta_{0}^{2}\right) \log ^{2}(\rho x)\right) .
$$

If $\theta_{0}^{2}=\theta_{x}^{2}$, then $\left(T^{-1} \Lambda_{0} T\right)_{21}=0$, and $x A_{0}(\rho, x), x A_{x}(\rho, x), A_{1}(\rho, x)-\Lambda_{1}$ admit representations $\sum_{n=1}^{\infty} x^{n} \sum_{m=0}^{n} A_{\iota m}^{0 n} \log ^{m}(\rho x)(\iota=0, x, 1)$. Using these facts, we obtain Theorem 2.4. 
Remark 6.2. If $\theta_{0}^{2}=\theta_{x}^{2}$, then, by Lemma 6.2 ,

$$
\begin{aligned}
& t^{\Lambda} \Lambda_{0} t^{-\Lambda}=T\left(\begin{array}{cc} 
\pm \theta_{0} / 2 & \mp \theta_{0} \log t \\
0 & \mp \theta_{0} / 2
\end{array}\right) T^{-1}, \\
& t^{\Lambda} \Lambda_{x} t^{-\Lambda}=T\left(\begin{array}{cc}
\mp \theta_{0} / 2 & 1 \pm \theta_{0} \log t \\
0 & \pm \theta_{0} / 2
\end{array}\right) T^{-1} .
\end{aligned}
$$

With these expressions in mind, observing the recursive relations in the proof of Proposition 5.6 and noting the logarithmic terms in the proof of Lemma 4.6, we can verify that, for (5.11) solving (5.1), the series $\kappa t A_{0}^{*}(t), \kappa t A_{x}^{*}(t), A_{1}^{*}(t)-\Lambda_{1}$ can be written in the form $\sum_{n=1}^{\infty} \sum_{m=0}^{n} A_{\iota m}^{* n}(\kappa t)^{n}\left(\theta_{0} \log t\right)^{m}$ with $A_{\iota m}^{* n} \in M_{2}\left(\mathbb{C}_{\theta}\right)$ $(\iota=0, x, 1)$.

\section{§6.3. Derivation of Corollary 2.5}

Suppose that $\theta_{x}^{2} \neq \theta_{0}^{2}$. Then from Theorem 2.4 it follows that

$$
\frac{x}{y_{0}(\rho, x)}=\frac{\left(1+F\left(x \log ^{2}(\rho x), \log (\rho x)\right)\right)^{-1}}{\log ^{2}(\rho x) F_{0}(\log (\rho x))}, \quad F(\tilde{x}, \xi)=\frac{1}{F_{0}(\xi)} \sum_{n=1}^{\infty} \tilde{x}^{n} F_{n}(\xi)
$$

with $F_{n}(\xi)=\xi^{-2(n+1)} P_{n+1}(\xi) \in \mathbb{C}_{\theta}\left[\xi^{-1}\right]$ and $F_{0}(\infty)=\left(\theta_{x}^{2}-\theta_{0}^{2}\right) / 4$. Since $y_{0}(\rho, x)$ converges absolutely in $\Omega^{*}\left(\varepsilon_{0}, \Theta_{0}\right)$, we have $c_{m}^{* n}=O\left(\left(\varepsilon_{0} / 2\right)^{-n}\right)$ for every $(m, n)$ such that $0 \leq m \leq 2 n$. Hence the function $\xi^{-2} F_{0}(\xi)^{-1}(1+F(\tilde{x}, \xi))^{-1}$ with

$$
F_{0}(\xi) F(\tilde{x}, \xi)=\sum_{n=1}^{\infty} \tilde{x}^{n} F_{n}(\xi)=\sum_{n=1}^{\infty} \sum_{m=0}^{2(n+1)} c_{m}^{*(n+1)} \tilde{x}^{n} \xi^{-(2(n+1)-m)}
$$

is holomorphic in $(\tilde{x}, \xi)$ such that $|\tilde{x}|<\tilde{x}_{0},|\xi|>\xi_{\infty}$, where $\tilde{x}_{0}$ is sufficiently small and $\xi_{\infty}$ is sufficiently large. Replacing $\left(\theta_{0}, \theta_{x}, \theta_{1}, \theta_{\infty}\right)$ by $\left(\theta_{\infty}-1, \theta_{1}, \theta_{x}, \theta_{0}+1\right)$, we derive the inverse logarithmic solution as in the corollary under the condition $\theta_{1}^{2} \neq\left(\theta_{\infty}-1\right)^{2}$. If $\theta_{x}^{2}=\theta_{0}^{2} \neq 0$, then

$$
\frac{x}{y_{0}(\rho, x)}=\frac{(1+\tilde{F}(x \log (\rho x), \log (\rho x)))^{-1}}{\log (\rho x) \tilde{F}_{0}(\log (\rho x))}, \quad \tilde{F}(\tilde{x}, \xi)=\frac{1}{\tilde{F}_{0}(\xi)} \sum_{n=1}^{\infty} \tilde{x}^{n} \tilde{F}_{n}(\xi)
$$

with $\tilde{F}_{n}(\xi)=\xi^{-(n+1)} P_{n+1}(\xi) \in \mathbb{C}_{\theta}\left[\xi^{-1}\right]$ and $\tilde{F}_{0}(\xi) \equiv \pm \theta_{0}$, which yields the desired solution under the condition $\theta_{1}= \pm\left(\theta_{\infty}-1\right) \neq 0$.

\section{§6.4. Remark on the choice of $\Lambda_{0}$ and $\Lambda_{x}$}

Let $\left(\Lambda_{0}, \Lambda_{x}, \Lambda, \Lambda_{1}, T\right)$ be as in Lemma 6.1. Set $\tilde{T}:=T r^{J / 2}$ and $\tilde{\Lambda}_{\iota}:=r^{\Lambda / \sigma} \Lambda_{\iota} r^{-\Lambda / \sigma}$ $(\iota=0, x)$ with $r \in \mathbb{C} \backslash\{0\}$. Then $\left(\tilde{\Lambda}_{0}, \tilde{\Lambda}_{x}, \Lambda, \Lambda_{1}, \tilde{T}\right)$ also satisfies (P.1) through (P.3). In discussing the relation between integration constants and monodromy data of the related Fuchsian system, such a choice of matrices is made (see [12], 
[4], [5]). Let $\left(\tilde{A}_{0}, \tilde{A}_{x}, \tilde{A}_{1}\right)$ be the solution given by Theorem 2.1 corresponding to $\left(\tilde{\Lambda}_{0}, \tilde{\Lambda}_{x}, \Lambda, \Lambda_{1}, \tilde{T}\right)$ with $\rho=1$. We set

$$
\tilde{A}_{0}=x^{\Lambda}\left(\tilde{\Lambda}_{0}+\tilde{U}_{0}\right) x^{-\Lambda}, \quad \tilde{A}_{x}=x^{\Lambda}\left(\tilde{\Lambda}_{x}+\tilde{U}_{x}\right) x^{-\Lambda}, \quad \tilde{A}_{1}=\Lambda_{1}+\tilde{U}_{1} .
$$

By Proposition 5.1, $\left(\tilde{U}_{0}, \tilde{U}_{x}, \tilde{U}_{1}\right)$ satisfies

$$
\begin{aligned}
& x \frac{d \tilde{U}_{0}}{d x}=-\left[x^{-\Lambda} \tilde{U}_{1} x^{\Lambda}, \tilde{\Lambda}_{0}+\tilde{U}_{0}\right], \\
& x \frac{d \tilde{U}_{x}}{d x}=-\left[x^{-\Lambda} \tilde{U}_{1} x^{\Lambda}, \tilde{\Lambda}_{x}+\tilde{U}_{x}\right]-\frac{x}{1-x}\left[x^{-\Lambda}\left(\Lambda_{1}+\tilde{U}_{1}\right) x^{\Lambda}, \tilde{\Lambda}_{x}+\tilde{U}_{x}\right], \\
& x \frac{d \tilde{U}_{1}}{d x}=-\frac{x}{1-x}\left[x^{\Lambda}\left(\tilde{\Lambda}_{x}+\tilde{U}_{x}\right) x^{-\Lambda}, \Lambda_{1}+\tilde{U}_{1}\right] .
\end{aligned}
$$

Since $x^{\Lambda} \tilde{\Lambda}_{\iota} x^{-\Lambda}=\left(r^{1 / \sigma} x\right)^{\Lambda} \Lambda_{\iota}\left(r^{1 / \sigma} x\right)^{-\Lambda}(\iota=0, x)$, the triple

$$
\left(U_{0}, U_{x}, U_{1}\right)=\left(r^{-\Lambda / \sigma} \tilde{U}_{0} r^{\Lambda / \sigma}, r^{-\Lambda / \sigma} \tilde{U}_{x} r^{\Lambda / \sigma}, \tilde{U}_{1}\right)
$$

solves (5.2) with $\kappa=r^{-1 / \sigma}, t=r^{1 / \sigma} x$. Then $\tilde{A}_{\iota}=\left(r^{1 / \sigma} x\right)^{\Lambda}\left(\Lambda_{\iota}+U_{\iota}\right)\left(r^{1 / \sigma} x\right)^{-\Lambda}$ $(\iota=0, x)$, which implies $\left(\tilde{A}_{0}, \tilde{A}_{x}, \tilde{A}_{1}\right) \equiv\left(A_{0}(\sigma, r, x), A_{x}(\sigma, r, x), A_{1}(\sigma, r, x)\right)$.

For $\left(\Lambda_{0}, \Lambda_{x}, \Lambda, \Lambda_{1}, T\right)$ as in Lemma $6.2,\left(\tilde{\Lambda}_{0}, \tilde{\Lambda}_{x}, \Lambda, \Lambda_{1}, \tilde{T}\right)$ with $\tilde{\Lambda}_{\iota}=e^{r \Lambda} \Lambda_{\iota} e^{-r \Lambda}$ $(\iota=0, x), \tilde{T}=T e^{r \Delta}$ also satisfies (P.1), (P.2'), (P.3). Then the solution given by Theorem 2.2 corresponding to $\left(\tilde{\Lambda}_{0}, \tilde{\Lambda}_{x}, \Lambda, \Lambda_{1}, \tilde{T}\right)$ with $\rho=1$ coincides with $\left(A_{0}\left(e^{r}, x\right), A_{x}\left(e^{r}, x\right), A_{1}\left(e^{r}, x\right)\right)$.

\section{Appendix}

Let $\sigma, \rho \in \mathbb{C} \backslash\{0\}$ and $r_{0}, \omega \in \mathbb{R}$ be such that $\operatorname{Im} \sigma \neq 0,0<\omega<1$. Then $\left|\rho x^{1+\sigma}\right|=O\left(|x|^{\omega}\right)$ along the curve $\Gamma\left(r_{0}, \omega\right)_{\sigma}:(1+\operatorname{Re} \sigma-\omega) \log |x|-\operatorname{Im} \sigma \cdot \arg x=r_{0}$. Let $y(x)$ be a solution of (PVI) such that

$$
y(x)=\rho x^{1+\sigma}(1+o(1)), \quad x y^{\prime}(x)=\rho x^{1+\sigma}(1+\sigma+o(1))
$$

as $x \rightarrow 0$ along $\Gamma\left(r_{0}, \omega\right)_{\sigma}$. By $y=\left(e^{u / 2}+e^{-u / 2}\right)^{-2}=e^{-u}\left(1+e^{-u}\right)^{-2}$, (PVI) is changed into

$$
x\left(x u^{\prime}\right)^{\prime}=f_{0}\left(x, e^{-u}, x e^{u}\right)+f_{1}\left(x, e^{-u}, x e^{u}\right)\left(x u^{\prime}\right)+f_{2}\left(x, e^{-u}, x e^{u}\right)\left(x u^{\prime}\right)^{2}
$$

$\left(u^{\prime}=d u / d x\right)$, where $f_{j}(x, \xi, \eta)(j=0,1,2)$ are holomorphic for $|x|,|\xi|,|\eta|<1 / 4$ and satisfy $f_{j}(0,0,0)=0$ (cf. [17, Proposition 5.1]). The further transformation

$$
u=-(1+\sigma) \log x-\log \rho-v, \quad x u^{\prime}=-(1+\sigma)-x v^{\prime}
$$

leads us to 


$$
\begin{aligned}
x\left(x v^{\prime}\right)^{\prime}= & f_{0}\left(x, \rho x^{1+\sigma} e^{v}, \rho^{-1} x^{-\sigma} e^{-v}\right) \\
& -f_{1}\left(x, \rho x^{1+\sigma} e^{v}, \rho^{-1} x^{-\sigma} e^{-v}\right)\left(1+\sigma+x v^{\prime}\right) \\
& +f_{2}\left(x, \rho x^{1+\sigma} e^{v}, \rho^{-1} x^{-\sigma} e^{-v}\right)\left(1+\sigma+x v^{\prime}\right)^{2} \\
= & F_{0}\left(x, \rho x^{1+\sigma}, \rho^{-1} x^{-\sigma}\right)+F\left(x, \rho x^{1+\sigma}, \rho^{-1} x^{-\sigma}, v, x v^{\prime}\right) .
\end{aligned}
$$

Here $F_{0}(x, \xi, \eta)$ and $F(x, \xi, \eta, v, w)$ are holomorphic for $|x|,|\xi|,|\eta|<1 /(8 e)$ and $|v|,|w|<1$, and satisfy

$$
\begin{aligned}
& F_{0}(x, \xi, \eta)=O(|x|+|\xi|+|\eta|), \\
& F(x, \xi, \eta, \tilde{v}, \tilde{w})-F(x, \xi, \eta, v, w)=O(|x|+|\xi|+|\eta|)(|\tilde{v}-v|+|\tilde{w}-w|)
\end{aligned}
$$

whenever $|v|,|w|,|\tilde{v}|,|\tilde{w}|<1$. Let $v(x)$ be the solution of (A.2) corresponding to $y(x)$. Along $\Gamma\left(r_{0}, \omega\right)_{\sigma}$,

$$
\begin{aligned}
& e^{v(x)}\left(1+\rho x^{1+\sigma} e^{v(x)}\right)^{-2}=e^{v(x)}\left(1+O\left(|x|^{\omega}\right) e^{v(x)}\right)^{-2}=1+o(1), \\
& \left(1+\sigma+x v^{\prime}(x)\right)\left(1-\frac{2 \rho x^{1+\sigma} e^{v(x)}}{1+\rho x^{1+\sigma} e^{v(x)}}\right) \\
& =\left(1+\sigma+x v^{\prime}(x)\right)\left(1+\frac{O\left(|x|^{\omega}\right) e^{v(x)}}{1+O\left(|x|^{\omega}\right) e^{v(x)}}\right)=1+\sigma+o(1),
\end{aligned}
$$

since $x y^{\prime} / y=-\left(1-2 e^{-u} /\left(1+e^{-u}\right)\right) x u^{\prime}$. Then we have $v(x), x v^{\prime}(x)=o(1)$ as $x \rightarrow 0$ along $\Gamma\left(r_{0}, \omega\right)_{\sigma}$. Observing that $\left|\rho x^{1+\sigma}\right|,\left|\rho^{-1} x^{-\sigma}\right|=O\left(|x|^{\omega}+|x|^{1-\omega}\right)$ along $\Gamma\left(r_{0}, \omega\right)_{\sigma}$ and using $v(x), x v^{\prime}(x)=o(1)$, we deduce that $v(x)$ satisfies the system of integral equations

$$
\begin{aligned}
x v^{\prime}(x) & =\int_{\Gamma(x)}\left(F_{0}\left(t, \rho t^{1+\sigma}, \rho^{-1} t^{-\sigma}\right)+F\left(t, \rho t^{1+\sigma}, \rho^{-1} t^{-\sigma}, v(t), t v^{\prime}(t)\right)\right) \frac{d t}{t}, \\
v(x) & =\int_{\Gamma(x)} \int_{\Gamma(s)}\left(F_{0}\left(t, \rho t^{1+\sigma}, \rho^{-1} t^{-\sigma}\right)+F\left(t, \rho t^{1+\sigma}, \rho^{-1} t^{-\sigma}, v(t), t v^{\prime}(t)\right)\right) \frac{d t}{t} \frac{d s}{s}
\end{aligned}
$$

with $\Gamma(x) \subset \Gamma\left(r_{0}, \omega\right)_{\sigma}$ joining 0 to $x$. Suppose that $v_{1}(x)$ and $v_{2}(x)$ solve this system and set $\phi(x):=\sup _{t \in \Gamma(x)}\left(\left|v_{2}(t)-v_{1}(t)\right|+\left|t v_{2}^{\prime}(t)-t v_{1}^{\prime}(t)\right|\right)$. Then using (A.3) and (A.4) we derive $\phi(x)=O\left(|x|^{\omega}+|x|^{1-\omega}\right) \phi(x)$, and hence $\phi(x) \equiv 0$. This implies the uniqueness of $v(x)$, from which that of $y(x)$ follows. The uniqueness under the condition $y(x) \sim \rho x^{1+\sigma}$ only may be proved by using the RiemannHilbert correspondence [4, Proposition in §4], [5, Proposition A.1].

\section{Acknowledgements}

The author expresses his deep gratitude to the referee for valuable comments, which were very helpful in revising this paper. Motivated by the referee's suggestion on the behaviour of $y(\sigma, \rho, x)$, the author obtained the results in Section 2.2. 


\section{References}

[1] A. D. Bryuno and I. V. Goryuchkina, All the asymptotic expansions of solutions of the sixth Painlevé equation, Dokl. Akad. Nauk 417 (2007), 298-302 (in Russian); English transl.: Dokl. Math. 76 (2007), 851-855. MR 2455353

[2] B. Dubrovin and M. Mazzocco, Monodromy of certain Painlevé-VI transcendents and reflection groups, Invent. Math. 141 (2000), 55-147. Zbl 0960.34075 MR 1767271

[3] I. V. Goryuchkina, Basic asymptotic expansions of solutions of the sixth Painlevé equation, Sovrem. Mat. Prilozh. 57 (2008), 3-9 (in Russian); English transl.: J. Math. Sci. (N. Y.) 160 (2009), 1-9. Zbl 1196.34114 MR 2676334

[4] D. Guzzetti, On the critical behavior, the connection problem and the elliptic representation of a Painlevé VI equation, Math. Phys. Anal. Geom. 4 (2001), 293-377. Zbl 1008.34081 MR 1888007

[5] The elliptic representation of the general Painlevé VI equation, Comm. Pure Appl. Math. 55 (2002), 1280-1363. Zbl 1047.34103 MR 1912098

[6] _ Matching procedure for the sixth Painlevé equation, J. Phys. A 39 (2006), 1197312031. Zbl 1133.34048 MR 2266210

[7] The logarithmic asymptotics of the sixth Painlevé equation, J. Phys. A 41 (2008), 205201, 46 pp. Zbl 1161.34062 MR 2450511

[8] _ Solving the sixth Painlevé equation: towards the classification of all the critical behaviors and the connection formulae, Int. Math. Res. Notices 2012, 1352-1413. Zbl 1246.34084 MR 2899955

[9] _ Tabulation of Painlevé 6 transcendents, Nonlinearity 25 (2012), 3235-3276. Zbl 1267.34152 MR 2993052

[10] 2188-2203. Zbl 06132976 MR 2998122

[11] M. Iwano, Analytic expressions for bounded solutions of nonlinear ordinary differential equations with Briot-Bouquet type singularity, Funkcial. Ekvac. 12 (1969), 41-88. Zbl 0222.34007 MR 0254323

[12] M. Jimbo, Monodromy problem and the boundary condition for some Painlevé equations, Publ. RIMS Kyoto Univ. 18 (1982), 1137-1161. Zbl 0535.34042 MR 0688949

[13] M. Jimbo and T. Miwa, Monodromy preserving deformation of linear ordinary differential equations with rational coefficients. II, Phys. D 2 (1981), 407-448. Z Zl 1194.34166 MR 0625446

[14] M. Mazzocco, Picard and Chazy solutions to the Painlevé VI equation, Math. Ann. 321 (2001), 157-195. Zbl 0999.34079 MR 1857373

[15] K. Okamoto, Studies on the Painlevé equations. I. Sixth Painlevé equation $P_{\text {VI }}$, Ann. Mat. Pura Appl. 146 (1987), 337-381. Zbl 0637.34019 MR 0916698

[16] M. Sato, T. Miwa and M. Jimbo, Holonomic quantum fields. II. The Riemann-Hilbert problem, Publ. RIMS Kyoto Univ. 15 (1979), 201-278. Z Zbl 0433.35058 MR 0533348

[17] S. Shimomura, A family of solutions of a nonlinear ordinary differential equation and its application to Painlevé equations (III), (V) and (VI), J. Math. Soc. Japan 39 (1987), 649662. Zbl 0638.34004 MR 0905630

[18] M. Yoshida and K. Takano, On a linear system of Pfaffian equations with regular singular points, Funkcial. Ekvac. 19 (1976), 175-189. Zbl 0352.35027 MR 0436189 